\title{
Development of Fluorine-18 Labeled Metabolically Activated Tracers for Imaging of Drug Efflux Transporters with PET
}

Kerstin Sander, Eva Galante, Thibault Gendron, Elena Yiannaki, Niral Patel, Tammy L Kalber, Adam Badar, Mathew Robson, Sean P Johnson, Florian Bauer, Severin Mairinger, Johann Stanek, Thomas Wanek, Claudia Kuntner, Tim Kottke, Lilia Weizel, David Dickens, Kjell Erlandsson, Brian F Hutton, Mark F Lythgoe, Holger Stark, Oliver Langer, Matthias Koepp, and Erik Årstad

J. Med. Chem., Just Accepted Manuscript • DOI: 10.1021/acs.jmedchem.5b00652 • Publication Date (Web): 10 Jul 2015

Downloaded from http://pubs.acs.org on July 14, 2015

\section{Just Accepted}

"Just Accepted" manuscripts have been peer-reviewed and accepted for publication. They are posted online prior to technical editing, formatting for publication and author proofing. The American Chemical Society provides "Just Accepted" as a free service to the research community to expedite the dissemination of scientific material as soon as possible after acceptance. "Just Accepted" manuscripts appear in full in PDF format accompanied by an HTML abstract. "Just Accepted" manuscripts have been fully peer reviewed, but should not be considered the official version of record. They are accessible to all readers and citable by the Digital Object Identifier (DOI®). "Just Accepted" is an optional service offered to authors. Therefore, the "Just Accepted" Web site may not include all articles that will be published in the journal. After a manuscript is technically edited and formatted, it will be removed from the "Just Accepted" Web site and published as an ASAP article. Note that technical editing may introduce minor changes to the manuscript text and/or graphics which could affect content, and all legal disclaimers and ethical guidelines that apply to the journal pertain. ACS cannot be held responsible for errors or consequences arising from the use of information contained in these "Just Accepted" manuscripts. 


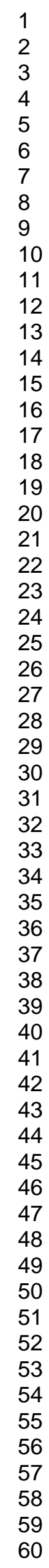

SCHOLARONE Manuscripts 


\section{Development of Fluorine-18 Labeled Metabolically}

\section{Activated Tracers for Imaging of Drug Efflux}

\section{Transporters with PET}

Kerstin Sander, ${ }^{1}$ Eva Galante, ${ }^{1}$ Thibault Gendron, ${ }^{1}$ Elena Yiannaki, ${ }^{2}$ Niral Patel, ${ }^{3}$ Tammy L. Kalber, ${ }^{3}$ Adam Badar, ${ }^{3}$ Mathew Robson, ${ }^{4}$ Sean P. Johnson, ${ }^{4}$ Florian Bauer, ${ }^{5}$ Severin Mairinger, ${ }^{6}$ Johann Stanek, ${ }^{6}$ Thomas Wanek, ${ }^{6}$ Claudia Kuntner, ${ }^{6}$ Tim Kottke, ${ }^{7}$ Lilia Weizel, ${ }^{7}$ David Dickens, ${ }^{8}$ Kjell Erlandsson, ${ }^{1}$ Brian F. Hutton, ${ }^{1}$ Mark F. Lythgoe, ${ }^{3}$ Holger Stark, ${ }^{7, \#}$ Oliver Langer, ${ }^{9}$ Matthias Koepp, ${ }^{10}$ Erik Arstad ${ }^{1,2, *}$

${ }^{1}$ University College London, Institute of Nuclear Medicine, 235 Euston Road - T5, London NW1 2BU, UK; ${ }^{2}$ University College London, Department of Chemistry, 20 Gordon Street, London WC1H 0AJ, UK; ${ }^{3}$ University College London, Centre for Advanced Biomedical Imaging, 72 Huntley Street, London WC1E 6DD, UK; ${ }^{4}$ University College London, Cancer Institute, 72 Huntley Street, London WC1E 6DD, UK; ${ }^{5}$ University of Vienna, Faculty of Life Sciences, Department of Medicinal Chemistry, Althanstrasse 14, A-1090 Vienna, Austria; ${ }^{6}$ AIT Austrian Institute of Technology $\mathrm{GmbH}$, Health and Environment Department, A-2444 Seibersdorf, Austria; ${ }^{7}$ Johann Wolfgang Goethe University, Institute of Pharmaceutical Chemistry, Biocenter, Max-von-Laue-Strasse 9, 60438 Frankfurt am Main, Germany; ${ }^{8}$ University of Liverpool, Institute of Translational Medicine, Department of Molecular and Clinical Pharmacology, The Wolfson Centre for Personalised Medicine, Block A Waterhouse 
Buildings, 1-5 Brownlow Street, Liverpool L69 3GL, UK; ${ }^{9}$ Medical University of Vienna, Department of Clinical Pharmacology, Waehringer-Guertel 18-20, A-1090 Vienna, Austria; ${ }^{10}$ University College London, Institute of Neurology, Department of Clinical and Experimental Epilepsy, Queen Square, London WC1N 3BG, UK 


\begin{abstract}
Increased activity of efflux transporters, e.g. P-glycoprotein (P-gp) and breast cancer resistance protein (BCRP), at the blood-brain barrier is a pathological hallmark of many neurological diseases, and the resulting multiple drug resistance represents a major clinical challenge. Noninvasive imaging of transporter activity can help to clarify the underlying mechanisms of drug resistance, and facilitate diagnosis, patient stratification and treatment monitoring. We have developed a metabolically activated radiotracer for functional imaging of P-gp/BCRP activity with positron emission tomography (PET). In preclinical studies, the tracer showed excellent initial brain uptake and clean conversion to the desired metabolite, although at a sluggish rate. Blocking with P-gp/BCRP modulators led to increased levels of brain radioactivity; however, dynamic PET did not show differential clearance rates between treatment and control groups. Our results provide proof-of-concept for development of pro-drug tracers for imaging of Pgp/BCRP function in vivo, but also highlight some challenges associated with this strategy.
\end{abstract}




\section{INTRODUCTION}

Membrane transporters at the blood-brain barrier (BBB) play an important role in maintaining this physiological barrier and hence, brain homeostasis: selective uptake transporters ensure the supply of essential nutrients like glucose, amino acids and nucleotides, whilst efflux transporters extrude toxic metabolites from the brain and prevent xenobiotics from reaching the central nervous system (CNS), thus providing a cellular detoxification system. The most prevalent efflux transporters at the $\mathrm{BBB}$ endothelium belong to the superfamily of adenosine triphosphatebinding cassette $(\mathrm{ABC})$ transporters, and include P-glycoprotein (P-gp; ABCB1) and breast cancer resistance protein (BCRP; ABCG2). ${ }^{1}$ P-gp and BCRP are located at the luminal membrane of the endothelial cells, where they extrude an array of structurally different compounds. Pharmacophore models developed for P-gp substrates suggest that compounds exhibiting two hydrogen bond acceptors, two aromatic rings and additional hydrophobic features are readily transported by the efflux pump. ${ }^{2}$ Recent research shows considerable overlap in substrate scope of P-gp and BCRP, ${ }^{3}$ and an increasing body of evidence suggests that they colocalize and cooperate in transporting compounds at the $\mathrm{BBB}^{4}$ Drug entry into the brain is therefore highly restricted, and is largely limited to compounds that are not recognized by efflux transporters and are able to cross the BBB by passive diffusion. In the course of many prevalent CNS disorders, especially refractory diseases like epilepsy, mood disorders and brain cancers, the delicate balance of transporter activity is distorted. The BBB becomes 'leaky', enabling toxic substances to temporarily reach the CNS, and, as a compensatory mechanism, activity and expression levels of efflux transporters increase. ${ }^{5}$ As a consequence of transporter upregulation at the $\mathrm{BBB}, \mathrm{CNS}$ drug concentrations in the brain become too low to achieve a therapeutic effect. The resulting multiple drug resistance (MDR) affects $20-40 \%$ of all patients suffering from 
epilepsy, depression and schizophrenia, and is even more prevalent in drug refractory brain cancer and following human immunodeficiency virus infections. ${ }^{6}$

Quantitative assessment of efflux pump function by positron emission tomography (PET) can help to clarify the underlying mechanisms of drug resistance in individual patients, and facilitate stratification of patients for specific treatment strategies. As P-gp/BCRP mediates extrusion of the most commonly prescribed CNS drugs, ${ }^{6 c, 7}$ a PET tracer that enables functional imaging of this transporter system can potentially be applicable to large patient cohorts spanning a range of diverse neurological diseases.

In recent years, a number of strategies for imaging of drug efflux transporters have been evaluated. Tracers that have been designed as inhibitors, e.g. $\left[{ }^{11} \mathrm{C}\right]$ tariquidar and analogues, often act as substrates when administered in the low doses that are required for imaging with PET. ${ }^{8}$ These P-gp/BCRP modulators, and P-gp substrates like $\left[{ }^{11} \mathrm{C}\right]$ verapamil all have significant limitations: poor metabolic stability, low brain uptake (substrates), low binding affinity (inhibitors), and insufficient selectivity make quantitative imaging of drug efflux pumps challenging. ${ }^{9}$ A more recent approach is the development of metabolically activated (pro-drug) tracers, as exemplified by carbon-11- and fluorine-18-labeled 6-halopurines for imaging of multidrug resistance protein $1 .^{10}$ The so-called metabolite extrusion method (MEM) relies on passive diffusion of a pro-drug tracer into the organ of interest, e.g. the brain, its subsequent trapping by metabolic conversion into an efflux pump substrate ('lock-in' mechanism), and transporter-mediated extrusion of the metabolite. ${ }^{11}$ Provided that the physicochemical properties of the metabolite preclude passive diffusion across membranes, drug efflux transporter function can be directly correlated to the clearance rate of radioactivity from the target tissue, and hence readily be quantified by dynamic imaging. In order to allow MEM to be exploited for 
quantitative imaging of $\mathrm{P}$-gp/BCRP function in vivo, a pro-drug tracer should have a high initial brain uptake and be rapidly transformed into a single radioactive metabolite that acts as a dual Pgp/BCRP substrate, but does not interact with other transporters or targets in the brain. The radioactive metabolite should be unable to cross membranes by passive diffusion, so that in the absence of P-gp and BCRP, it is effectively trapped. ${ }^{9 b}$

Histamine $\mathrm{H}_{1}$ receptor $\left(\mathrm{H}_{1} \mathrm{R}\right)$ antagonists are widely used for the treatment of allergies, and are often characterized by their ability to cross the BBB: compounds of the first generation readily enter the brain causing $\mathrm{H}_{1} \mathrm{R}$-mediated side effects, whereas substances of the second generation are avid P-gp substrates and hence give minimal exposure to the CNS. ${ }^{12}$ Interestingly, several $\mathrm{H}_{1} \mathrm{R}$ antagonists currently in clinical use are active metabolites of early generation drugs, e.g. cetirizine and fexofenadine are zwitterions formed by enzymatic oxidation of hydroxyzine and terfenadine, respectively. ${ }^{13}$ These well-characterized metabolic pairs of $H_{1} \mathrm{R}$ antagonists were used as templates for the design of radiolabeled pro-drug tracers and metabolically activated dual P-gp/BCRP substrates (Figure 1).

\section{RESULTS \& DISCUSSION}

Chemistry. Using the $\mathrm{H}_{1} \mathrm{R}$ antagonists fexofenadine and cetirizine as templates, a library of compounds was prepared (Scheme 1 and Scheme 2) in order to identify (i) structural motifs required for P-gp substrate activity, (ii) functionalities that could be exploited as pro-drug moieties for metabolic activation, and (iii) potential positions for labeling with fluorine-18 (Figure 1). With the aim to circumvent the need for complex synthetic routes, 1benzhydrylpiperazine (scaffold A, derived from cetirizine) and diphenyl(piperidin-4-yl)methanol (scaffold $\mathbf{B}$, derived from fexofenadine) derivatives decorated with benzylic and aliphatic side 
chains were initially evaluated. Scaffold $\mathbf{B}$ as well as its mono- and difluorinated analogues $\mathbf{C}$ and $\mathbf{D}$, which were obtained by Grignard reactions with para-substituted piperidine derivatives (vide infra), were reacted with suitably functionalized benzylic halides by means of $N$-alkylation to give compounds $\mathbf{1}, \mathbf{3}, \mathbf{6}, \mathbf{9}, \mathbf{1 2}, \mathbf{1 4}$, and $\mathbf{1 6}$ (Scheme 1$).{ }^{14}$ The alkylating reagents were either commercially available, or prepared from the respective tolyl derivatives by radical bromination with $N$-bromosuccinimide. The alcohol 1 was further modified by Swern oxidation to obtain aldehyde $2,{ }^{15}$ whereas hydrolysis of the esters $3, \mathbf{6}, \mathbf{9}, \mathbf{1 2}, \mathbf{1 4}$, and $\mathbf{1 6}$ under basic conditions gave the corresponding carboxylic acids $4,7,10,13,15$, and 17.

A fluorinated aliphatic side chain was prepared as depicted in Scheme 2. Treatment of $\alpha$ hydroxy- $\gamma$-butyrolactone with DAST provided the fluorinated lactone $\mathbf{1 8},{ }^{16}$ which after delactonization with methanol gave alcohol 19, albeit in moderate yield (20\%). ${ }^{17}$ The alcohol 19 was converted into the corresponding tosylate $\mathbf{2 0}$, which was used for subsequent $N$-alkylation of scaffolds $\mathbf{A}$ and $\mathbf{B} .^{18}$ The resulting esters 21 and $\mathbf{2 5}$ were either reduced to give alcohols 22 and $\mathbf{2 6},{ }^{19}$ or hydrolysed to yield the acids $\mathbf{2 4}$ and $\mathbf{2 8} .^{20}$ Finally, treatment of the alcohols $\mathbf{2 2}$ and $\mathbf{2 6}$ with acetic anhydride provided the acyl esters $\mathbf{2 3}$ and $\mathbf{2 7 .}$

As biodistribution studies with $\left[{ }^{18} \mathrm{~F}\right] \mathbf{2 3}$ and $\left[{ }^{18} \mathrm{~F}\right] 27$ pointed to rapid defluorination of the aliphatic side chain in vivo (vide infra), a second set of compounds was designed consisting of fluorinated aromatic scaffolds and aliphatic side chains carrying pro-drug groups (alcohol, aldehyde and ester) as putative tracer candidates. To enable tuning of lipophilicity, additional scaffolds (C-F) were prepared (Scheme 3). Treatment of Boc-protected piperidine-4-carboxylic acid ethyl ester with 4-fluorophenylmagnesium bromide gave the difluorinated compound 33 . $^{21}$ The corresponding mono-fluorinated analogue 32 was prepared from Boc-protected 4hydroxymethyl-piperidine using sequential oxidation and Grignard reactions. ${ }^{22}$ Deprotection of 
compounds 31-33 provided the mono- and difluorinated scaffolds $\mathbf{3 4}, \mathbf{3 5}$ and $\mathbf{3 7}$ (= scaffold C, D and $\mathbf{F}$ ), whereas treatment of $\mathbf{3 2}$ with trifluoroacetic acid resulted in elimination of the tertiary alcohol to give compound $\mathbf{3 6}$ (= scaffold $\mathbf{E}$ ). $N$-alkylation of scaffolds $\mathbf{C}, \mathbf{E}$ and $\mathbf{F}$ with appropriately functionalized alkyl bromides provided the putative pro-drug tracers and nonradioactive reference compounds 38-47 (Figure 2).

In order to enable labeling of the putative tracers $\mathbf{2 3}$ and 27 with fluorine-18, the corresponding mesylate precursors 52 and $\mathbf{5 6}$ were prepared from a dioxolane building block (Scheme 4). The tosylate $\mathbf{4 8}$ was coupled to scaffolds $\mathbf{A}$ and $\mathbf{B}$, and the resulting protected diols $\mathbf{4 9}$ and $\mathbf{5 3}$ were subsequently hydrolyzed under acidic conditions to give vicinal diols $\mathbf{5 0}$ and $\mathbf{5 4}$. Selective acetylation of the primary alcohol in compounds $\mathbf{5 0}$ and $\mathbf{5 4}$ was achieved with acetic anhydride to give the acetates $\mathbf{5 1}$ and $\mathbf{5 5}$, which upon mesylation gave the desired precursors $\mathbf{5 2}$ and $\mathbf{5 6}$, respectively.

Whereas labeling of aliphatic groups with fluorine-18 is well established, aromatic $\left[{ }^{18} \mathrm{~F}\right]$ fluorination of small molecules bearing complex functional groups remains a challenge. ${ }^{23}$ Limitations of existing labeling methods prompted us to explore the use of sulfonium salts ${ }^{24}$ as leaving groups for aromatic $\left[{ }^{18} \mathrm{~F}\right]$ fluorination of the scaffolds described above. ${ }^{25}$ For the synthesis of the sulfonium salts $\mathbf{6 2}$ and $\mathbf{6 4}$ bearing an electron deficient scaffold, the ketone 59 was prepared by a lithium-mediated reaction of bromoarene $\mathbf{5 7}$ and Weinreb amide $\mathbf{5 8}$ (Scheme 5). After Boc deprotection of 59, the desired side chains were introduced by $N$-alkylation, and the ammonium salts of the resulting diaryl thioethers $\mathbf{6 1}$ and $\mathbf{6 3}$ were treated with diphenyliodonium triflate in the presence of copper(II) benzoate to give the triarylsulfonium salts 62 and 64, respectively. Labeling of compounds with the electron neutral scaffold $\mathbf{E}$ required decoration of the sulfonium salts with anisole moieties in order to achieve regioselective 
$\left[{ }^{18} \mathrm{~F}\right]$ fluorination. The thioether $\mathbf{6 5}$ was converted into ketone $\mathbf{6 6}$ as described above. Addition of phenylmagnesium bromide to $\mathbf{6 6}$ provided alcohol 67, which upon treatment with trifluoroacetic acid yielded the deprotected 4-(methylene)piperidine 68. Following the route outlined above, $N$ alkylation of 68 gave compounds 69, 71 and 73, which were converted into the corresponding triarylsulfonium salts 70, 72 and $\mathbf{7 4}$ using bis-(4-methoxyphenyl)iodonium triflate $\mathbf{7 5}{ }^{26}$

Radiochemistry. Radiolabeling of the mesylate precursors 52 and 56 (Scheme 6) with $\left[{ }^{18} \mathrm{~F}\right]$ fluoride worked well in dimethyl sulfoxide under heating $\left(20 \mathrm{~min}\right.$ at $\left.90{ }^{\circ} \mathrm{C}\right)$. Acetyl esters $\left[{ }^{18} \mathrm{~F}\right] 23$ and $\left[{ }^{18} \mathrm{~F}\right] 27$ were obtained in $40-50 \%$ analytical radiochemical yield (RCY) as determined by radio-HPLC. The isolated products were radiochemically pure but the specific activity was low $(0.5 \mathrm{GBq} / \mu \mathrm{mol})$ due to extensive decomposition of the respective precursors. As attempted hydrolysis of $\left[{ }^{18} \mathrm{~F}\right] 23$ and $\left[{ }^{18} \mathrm{~F}\right] 27$ to the corresponding alcohols $\left[{ }^{18} \mathrm{~F}\right] 22$ and $\left[{ }^{18} \mathrm{~F}\right] 26$ further reduced the specific activity, the acetyl esters $\left[{ }^{18} \mathrm{~F}\right] 23$ and $\left[{ }^{18} \mathrm{~F}\right] 27$ were used for an initial biological evaluation of compounds $\left[{ }^{18} \mathrm{~F}\right] 22$ and $\left[{ }^{18} \mathrm{~F}\right] \mathbf{2 6}$ assuming that the esters would be rapidly cleaved by esterases in the blood after intravenous injection (Scheme 6).

$\left[{ }^{18} \mathrm{~F}\right]$ Fluorination of triarylsulfonium salts gave the highest radiochemical yields when using a combination of potassium bicarbonate, kryptofix and DMSO with heating to $110{ }^{\circ} \mathrm{C}$ for 15 min. ${ }^{25}$ In the case of substrates containing electron withdrawing groups on the aromatic moiety, i.e. the ketones 62 and 64 (Scheme 7), the radiochemical reaction was exceptionally clean and yielded $\left[{ }^{18} \mathrm{~F}\right] 45$ (36-40\% decay-corrected isolated $\left.\mathrm{RCY}, n=3\right)$ and $\left[{ }^{18} \mathrm{~F}\right] 47$ (22\% decaycorrected isolated RCY), respectively, without formation of any radioactive side products. Hydrolysis of $\left[{ }^{18} \mathrm{~F}\right] 45$ afforded the alcohol $\left[{ }^{18} \mathrm{~F}\right] 46$ (18\% decay-corrected isolated RCY after two steps), whereas treatment of $\left[{ }^{18} \mathrm{~F}\right] 45$ with phenylmagnesium bromide led to formation of the fexofenadine-like scaffold $\mathbf{C}$ with concurrent cleavage of the ester group to give the alcohol 
$\left[{ }^{18} \mathrm{~F}\right] 39$ (16\% decay-corrected isolated RCY after two steps). Subsequent reaction of $\left[{ }^{18} \mathrm{~F}\right] 39$ with acetyl chloride provided the acyl ester $\left[{ }^{18} \mathrm{~F}\right] \mathbf{3 8}$.

Compounds containing the non-activated ritanserin-like scaffold $\mathbf{E}$ were prepared by direct labeling of the respective sulfonium salts $\mathbf{7 0 , 7 2}$ and $\mathbf{7 4}$ with $\left[{ }^{18} \mathrm{~F}\right]$ fluoride (Scheme 8). The resulting esters $\left[{ }^{18} \mathrm{~F}\right] \mathbf{4 0 a}$ and $\left[{ }^{18} \mathrm{~F}\right] \mathbf{4 3}$ as well as the acetal $\left[{ }^{18} \mathrm{~F}\right] \mathbf{4 1}$ were hydrolyzed to give the alcohol $\left[{ }^{18} \mathrm{~F}\right] 40$, the carboxylic acid $\left[{ }^{18} \mathrm{~F}\right] 44$ and the aldehyde $\left[{ }^{18} \mathrm{~F}\right] 43$, respectively. The radiochemical purity of the desired radiotracers $\left(\left[{ }^{18} \mathrm{~F}\right] 38-\left[{ }^{18} \mathrm{~F}\right] \mathbf{4 0},\left[{ }^{18} \mathrm{~F}\right] \mathbf{4 2}-\left[{ }^{18} \mathrm{~F}\right] 44,\left[{ }^{18} \mathrm{~F}\right] 46\right.$ and $\left.\left[{ }^{18} \mathrm{~F}\right] 47\right)$ was $>96 \%$, however, the use of multi-step synthesis resulted in moderate overall yields (2-18\% decay-corrected isolated RCY after formulation in saline).

In our hands, preparation of sulfonium salts from the corresponding thioethers provided a practical and versatile synthetic method that gave rapid access to a number of structurally diverse precursors for labeling. Conveniently, triarylsulfonium salts are highly stable, easy to handle, and can readily be purified using conventional techniques including extractive aqueous workup and column chromatography on silica gel. In labeling studies, the precursors showed a remarkable reactivity with $\left[{ }^{18} \mathrm{~F}\right]$ fluoride: activated substrates reacted at room temperature, and with heating the reaction proceeded in the presence of hydrogen bond donors. Furthermore, efficient and regioselective $\left[{ }^{18} \mathrm{~F}\right]$ fluorination of non-activated aromatic moieties was achieved using sulfonium salts decorated with electron rich spectator ligands. The ability to label a spectrum of electron neutral to electron deficient aromatic groups with ${ }^{18} \mathrm{~F}$ in the presence of basic moieties, as well as hydrogen bond donors, makes the method broadly applicable to drug-like compounds, and hence opens up extensive pharmacological space for the design of small molecule PET tracers.

In vitro compound screening. A luminescent P-gp ATPase assay was initially used to establish if the simplified fexofenadine derivatives retained the P-gp substrate activity of the 
parent compounds, and to identify putative metabolic pairs that could be exploited for the design of a pro-drug tracer. The assay allows rapid screening of P-gp substrate affinity, but the accuracy is insufficient to determine $\mathrm{IC}_{50}$ values. ${ }^{27}$ In order to determine structural elements that are prerequisite to efflux pump substrate activity, ATP turnover triggered by the carboxylic acids $\mathbf{4}$, $\mathbf{7}$ and $\mathbf{1 0}$ was compared to that of parent compound fexofenadine (Figure 3A). Of the test compounds, the benzoic acid $\mathbf{4}$ evoked the highest ATPase activity, which was similar to that of fexofenadine, whereas ketone $\mathbf{7}$ and isobutyric acid $\mathbf{1 0}$ appeared to be poor P-gp substrates. To evaluate functional groups for the design of pro-drug tracers, the benzylic alcohol 1, benzaldehyde $\mathbf{2}$, and benzoic ester 3 were tested (Figure 3B). The results pointed to a gradual increase in P-gp affinity with increasing oxidation state, with alcohol 1 resulting in modest ATP consumption, whereas the aldehyde $\mathbf{2}$ and ester $\mathbf{3}$ appeared to be more avid substrates. Remarkably, incorporation of fluorine into the benzoic moiety of $\mathbf{4}$ to give compound $\mathbf{1 3}$ practically abolished the P-gp substrate affinity (Figure 3C). However, fluorine substituents on scaffold $\mathbf{B}$ were well tolerated, and the mono- and difluorinated derivatives $\mathbf{1 5}$ and $\mathbf{1 7}$ evoked ATP turnover comparable to that of compound 4. The use of a fluorinated aliphatic side chain, as for alcohol 22 and carboxylic acid 24, also appeared to be tolerated, and evoked ATP consumption to the same degree as their parent compounds hydroxyzine, and its metabolite cetirizine, respectively (Figure 3D). The results suggest that the zwitterions $\mathbf{4}$ and $\mathbf{2 4}$ are good Pgp substrates, whereas the corresponding alcohols $\mathbf{1}$ and $\mathbf{2 2}$ are likely to evade active efflux, and hence are suitable leads for pro-drug tracer development.

Tracer Evaluation in Vivo. The putative pro-drug tracers were evaluated using biodistribution studies in mice. The tissue distribution of radioactivity at 5 and $30 \mathrm{~min}$ post-injection (p.i.) was used to assess brain uptake, brain/blood ratios (Table 1), and the rate of clearance. Two 
derivatives labeled at the aliphatic side chain, namely $\left[{ }^{18} \mathrm{~F}\right] \mathbf{2 3}$ (cetirizine-like scaffold $\mathbf{A}$ ) and $\left[{ }^{18} \mathrm{~F}\right] 27$ (fexofenadine-like scaffold $\mathbf{B}$ ), were initially investigated to guide the choice of scaffold. As discussed above, we envisaged that ester hydrolysis would lead to rapid formation of the resulting alcohols $\left[{ }^{18} \mathrm{~F}\right] 22$ and $\left[{ }^{18} \mathrm{~F}\right] 26$ in vivo. Administration of $\left[{ }^{18} \mathrm{~F}\right] 23$ and $\left[{ }^{18} \mathrm{~F}\right] 27$ resulted in good initial uptake of radioactivity in the brain (5.8 and 7.2\% injected dose per gram tissue (ID/g) at $5 \mathrm{~min}$ p.i., respectively), however, high uptake (3-6\% ID/g at $30 \mathrm{~min}$ p.i.) was also observed in bone tissue (femur and skull) suggesting rapid enzymatic defluorination in vivo. Hence, we abandoned further evaluation of derivatives containing fluorinated aliphatic side chains, and instead evaluated pro-drug tracers bearing fluorine-18 at aromatic moieties. Indeed, administration of the acetate ester $\left[{ }^{18} \mathrm{~F}\right] \mathbf{3 8}$, a structurally closely related analogue to $\left[{ }^{18} \mathrm{~F}\right] \mathbf{2 7}$, exhibited far superior C-F bond stability as judged by the low level of radioactivity in bone tissue $(<2 \% \mathrm{ID} / \mathrm{g}$ at $30 \mathrm{~min}$ p.i. $)$. However, the brain uptake and brain/blood ratio of alcohol $\left[{ }^{18} \mathrm{~F}\right] 39$, as well as the corresponding acetyl ester $\left[{ }^{18} \mathrm{~F}\right] \mathbf{3 8}$, were low. To improve the initial uptake of the prodrug tracer in the brain, we prepared an analogue of $\left[{ }^{18} \mathrm{~F}\right] 39$ for which the hydroxyl group in scaffold $\mathbf{B}$ was replaced by an alkene to give scaffold $\mathbf{E}$. The resulting pro-drug tracer $\left[{ }^{18} \mathrm{~F}\right] \mathbf{4 0}$ exhibited excellent initial brain uptake $(9.4 \% \mathrm{ID} / \mathrm{g}$ at $5 \mathrm{~min}$ p.i.) with high $\mathrm{brain} / \mathrm{blood}$ ratios (10.4 and 7.3 at 5 and 30 min p.i., respectively). In contrast, administration of the corresponding carboxylic acid $\left[{ }^{18} \mathrm{~F}\right] 44$ resulted in an initial brain uptake of $1.3 \% \mathrm{ID} / \mathrm{g}$ at $5 \mathrm{~min}$ p.i. with a brain/blood ratio of 0.4 . In accordance with the results from the in vitro assay, replacement of the alcohol with an aldehyde $\left(\left[{ }^{18} \mathrm{~F}\right] \mathbf{4 2}\right)$ or ester $\left(\left[{ }^{18} \mathrm{~F}\right] \mathbf{4 3}\right)$ as the pro-drug group proved futile, and resulted in moderate brain uptake (3.1 and $2.9 \mathrm{ID} / \mathrm{g}$ at $5 \mathrm{~min}$ p.i., respectively). Alcohol $\left[{ }^{18} \mathrm{~F}\right] 46$, derived from scaffold $\mathbf{F}$, showed good initial brain uptake $(5.0 \% \mathrm{ID} / \mathrm{g}$ at $5 \mathrm{~min}$ p.i.), but brain clearance was rapid $\left(1.8 \% \mathrm{ID} / \mathrm{g}\right.$ at $30 \mathrm{~min}$ p.i.). The structurally related ester $\left[{ }^{18} \mathrm{~F}\right] 47$ hardly 
penetrated the brain, most likely due to the rapid hydrolysis in vivo to give the corresponding carboxylic acid (70\% conversion at $5 \mathrm{~min}$ p.i.).

The 4-hydroxybutyl pro-drug tracer $\left[{ }^{18} \mathrm{~F}\right] \mathbf{4 0}$ showed high initial brain uptake and a favorable clearance from the brain and was thus investigated further (Figure 4). The brain metabolism was exceptionally clean and gave the desired carboxylic acid $\left[{ }^{18} \mathrm{~F}\right] 44$ as the only radioactive metabolite. The ratio of $\left[{ }^{18} \mathrm{~F}\right] \mathbf{4 0}$ to $\left[{ }^{18} \mathrm{~F}\right] \mathbf{4 4}$ in the brain decreased over time and was determined to be 6.1 at $30 \mathrm{~min}$ p.i., 1.5 at $60 \mathrm{~min}$ p.i., 0.6 at $90 \mathrm{~min}$ p.i. and 0.4 at $120 \mathrm{~min}$ p.i., respectively. However, as $\left[{ }^{18} \mathrm{~F}\right] \mathbf{4 4}$ is extruded from the brain into the periphery, this ratio does not reflect the actual rate of metabolism. In the blood, oxidation of the alcohol was faster: whilst low amounts of $\left[{ }^{18} \mathrm{~F}\right] \mathbf{4 0}$ were still detectable, $\left[{ }^{18} \mathrm{~F}\right] \mathbf{4 4}$ constituted between 70 and $85 \%$ of total amount of radioactivity detected between 60 and 120 min p.i. (Figure 4C/D). Development of a MEM tracer requires that the radioactive metabolite does not cross the BBB to any significant extent by other mechanisms than extrusion by the targeted efflux pump. As the brain/blood ratios observed after administration of the carboxylic acid $\left[{ }^{18} \mathrm{~F}\right] 44$ remained at or below 1.1 over the course of two hours after administration (Figure 4B), our results suggest that brain clearance from $\left[{ }^{18} \mathrm{~F}\right] 44$ is largely mediated by active efflux. However, a faster metabolism of the pro-drug tracer $\left[{ }^{18} \mathrm{~F}\right] \mathbf{4 0}$ would be desirable. The aldehyde $\left[{ }^{18} \mathrm{~F}\right] 42$ and ester $\left[{ }^{18} \mathrm{~F}\right] 43$ were designed as to accelerate formation of the carboxylic acid $\left[{ }^{18} \mathrm{~F}\right] 44$ in vivo. Although metabolism to the zwitterions $\left[{ }^{18} \mathrm{~F}\right] 44$ was completed within five (ester) and 60 (aldehyde) minutes, respectively, their low brain/blood ratios rendered them unsuitable as pro-drug tracers ( $c f$. Table 1).

The pro-drug tracer $\left[{ }^{18} \mathrm{~F}\right] \mathbf{4 0}$ was investigated further to determine if the corresponding metabolite $\left[{ }^{18} \mathrm{~F}\right] \mathbf{4 4}$ acted as an efflux pump substrate in vivo. By injecting the dual P-gp/BCRP inhibitor tariquidar one to two hours prior to tracer administration at a dose of $15 \mathrm{mg} / \mathrm{kg}$ 
bodyweight it was ensured that P-gp was fully blocked over the course of the biodistribution studies (longest time point 120 min p.i.). ${ }^{28}$ The tariquidar dose is, however, not sufficiently high to fully inhibit BCRP. In a separate experiment, injection of elacridar (10 mg/kg bodyweight) was used to completely block both P-gp and BCRP. ${ }^{8 c}$ The comparison of the organ distribution without and with concomitant administration of tariquidar and elacridar, respectively (Figure 5A), showed a similar brain uptake at $30 \mathrm{~min}$ p.i. (ca. $8 \% \mathrm{ID} / \mathrm{g}$ ) in all animals, but revealed a significant effect on efflux pump inhibition at 60-120 min p.i.: when blocking with tariquidar, tracer brain uptake (in $\% \mathrm{ID} / \mathrm{g}$ ) at 60 and 90 min p.i. was 30 and $50 \%$ higher than in untreated animals. Using elacridar, the amount of tracer in the brain was doubled between 60 and $120 \mathrm{~min}$ p.i. Tracer levels in the blood remained constant between 1 and $2 \% \mathrm{ID} / \mathrm{g}$ in all animals. The results provide evidence for active transport of the metabolically activated tracer $\left[{ }^{18} \mathrm{~F}\right] 44$ across the BBB by P-gp as well as BCRP.

Dynamic PET scans were performed in untreated mice, mice treated with either tariquidar or elacridar, and P-gp knockout mice (Figure 5B/C). Whole brain time-activity curves were fitted with the sum of a constant (irreversible compartment, representing the amount of tracer trapped in the brain) and a mono-exponential function (reversible compartment, representing the amount of tracer washed out from the brain and the efflux rate). ${ }^{29}$ For analysis and determination of efflux rates, data from 10 min p.i. onwards was used as this gave the best match between the fitted curves and the experimental results. Focusing on the time from 60 min p.i. onwards, i.e. when increasing formation of the metabolite $\left[{ }^{18} \mathrm{~F}\right] \mathbf{4 4}$ was observed, did not change the quantitative outcome measure.

In a first set of experiments (performed at AIT), untreated, tariquidar treated and P-gp knockout mice (Figure 5B) showed comparable initial brain uptake. The ratio of tracer that was 
washed out from the brain to tracer that was trapped (washout/trapped ratio) was similar in untreated and knockout mice (2.9 and 2.8, respectively), whereas in tariquidar treated mice, the washout/trapped ratio was lower (1.5). This suggests that more tracer was trapped in the brains of the treated mice. In a second set of experiments (performed at UCL), elacridar treated mice were compared to untreated animals and showed a lower washout/trapped ratio (2.3 versus 4.1). The marginally higher peak brain uptake in elacridar treated mice compared to the control group may have contributed to the increased amounts of radioactivity at later time points (Figure 5C). It should be noted that discrepancies between the two control groups (washout/trapped ratio of 1.5 and 2.3, respectively) are most likely due to variations in the experimental setup. The difference in washout/trapped ratios provides evidence for $\left[{ }^{18} \mathrm{~F}\right] 44$ acting as a dual P-gp/BCRP substrate, and the experiments in P-gp knockout mice suggest that BCRP can compensate for P-gp when extruding $\left[{ }^{18} \mathrm{~F}\right] 44$. However, minor differences in efflux rates when comparing untreated and treated mice raise the possibility that not only tracer $\left[{ }^{18} \mathrm{~F}\right] \mathbf{4 4}$ but also the pro-drug tracer $\left[{ }^{18} \mathrm{~F}\right] \mathbf{4 0}$ was washed out of the brain, either by passive diffusion or by active transport as an efflux pump substrate.

Due to the complimentary and partially overlapping substrate scope of efflux pumps belonging to the large family of $\mathrm{ABC}$ transporters it is possible that transporters other than $\mathrm{P}$-gp/BCRP contribute to the efflux of $\left[{ }^{18} \mathrm{~F}\right] \mathbf{4 0} /\left[{ }^{18} \mathrm{~F}\right] 44 .{ }^{30}$ The tracer kinetics can also be affected by other factors such as off-target binding. Indeed, both the alcohol $\mathbf{4 0}$ and the carboxylic acid $\mathbf{4 4}$ showed high affinity to the human $\mathrm{H}_{1} \mathrm{R}(2.3 \pm 0.4$ and $18 \pm 4 \mathrm{nM}$, respectively) as determined in an in vitro ligand displacement assay. The affinity to $\mathrm{H}_{1} \mathrm{R}$ may contribute to the retention of the prodrug tracer $\left[{ }^{18} \mathrm{~F}\right] \mathbf{4 0}$ in the brain and may affect the rate of oxidative metabolism. Moreover, the metabolic activation, i.e. the conversion of $\left[{ }^{18} \mathrm{~F}\right] \mathbf{4 0}$ to $\left[{ }^{18} \mathrm{~F}\right] \mathbf{4 4}$, appeared to be slower than the 
extrusion of the metabolite $\left[{ }^{18} \mathrm{~F}\right] \mathbf{4 4}$, and as a result there was no significant difference between the brain clearance rates of the control group and the two treatment groups. However, differences in the oxidizing rate of enzymes, as well as in efflux transporter distribution and function, may make $\left[{ }^{18} \mathrm{~F}\right] \mathbf{4 0}$ more suitable as a pro-drug tracer in other species. ${ }^{31}$

\section{SUMMARY \& CONCLUSIONS}

With the aim to develop a pro-drug tracer to enable dynamic imaging of P-gp/BCRP function in vivo, we used $\mathrm{H}_{1} \mathrm{R}$ antagonists as lead compounds to synthesize a library of putative pro-drug tracers and their corresponding metabolites. In biodistribution studies, derivatives containing a fluorinated aliphatic side chain were found to undergo rapid defluorination in vivo. The results prompted us to develop triarylsulfonium salts as novel precursors for aromatic labeling of druglike molecules with fluorine-18. This labeling strategy provided access to a range of putative pro-drug tracers and corresponding metabolites, and allowed identification of the alcohol $\left[{ }^{18} \mathrm{~F}\right] \mathbf{4 0}$ as a lead pro-drug tracer candidate. $\left[{ }^{18} \mathrm{~F}\right] \mathbf{4 0}$ fulfills many of the requirements of MEM: it has excellent initial brain uptake (9.4\% ID/g 5 min p.i.) and undergoes clean metabolic conversion to the desired carboxylate $\left[{ }^{18} \mathrm{~F}\right] \mathbf{4 4}$ in the brain. Blocking experiments with the dual P-gp/BCRP inhibitors tariquidar and elacridar led to significantly increased levels of radioactivity in the brain in a period between 60 and 120 minutes after tracer injection, suggesting that the metabolite $\left[{ }^{18} \mathrm{~F}\right] 44$ is a substrate for both P-gp and BCRP. However, there was no significant difference in the brain efflux rates between control animals and treatment groups as measured by dynamic PET. Because of the sluggish metabolic conversion and cross-reactivity with $\mathrm{H}_{1} \mathrm{R}$ we were not able to use $\left[{ }^{18} \mathrm{~F}\right] \mathbf{4 0} /\left[{ }^{18} \mathrm{~F}\right] \mathbf{4 4}$ for quantification of the efflux rate; however, the tracer is still likely to provide a qualitative depiction of regional brain efflux rates. 
Our results provide proof-of-concept for development of metabolically activated pro-drug tracers for imaging of P-gp/BCRP activity with PET. However, the results also highlight some of the challenges in achieving appropriate rates of enzymatic conversion and brain clearance.

\section{EXPERIMENTAL SECTION}

Chemistry. Reagents were purchased from Sigma-Aldrich, Acros Organics or Fluorochem and were used without further purification. Purification of non-labeled compounds by column chromatography was performed on silica unless otherwise specified. For characterization of nonlabeled compounds, ${ }^{1} \mathrm{H}$ and ${ }^{13} \mathrm{C}$ NMR spectra were recorded at room temperature unless otherwise specified. The respective instruments, Bruker Avance 400, 500 or 600 were operated at a frequency of 400,500 or $600 \mathrm{MHz}$ for ${ }^{1} \mathrm{H}$ and 125 or $150 \mathrm{MHz}$ for ${ }^{13} \mathrm{C}$, respectively. Proton decoupled ${ }^{19}$ F NMR spectra were recorded on a Bruker Avance 300 instrument at a frequency of $282 \mathrm{MHz}$. All spectra were internally referenced to the respective deuterated solvents. Chemical shifts are reported in ppm and coupling constants $(J)$ are given in Hertz $(\mathrm{Hz})$. Full NMR assignment was performed with the aid of multidimensional and long range experiments. High resolution mass data were recorded on either a Thermo Finnigan MAT900xp (CI, EI) or a MALDI microMX (TOF) mass spectrometer. Melting points were taken on a Gallenkamp heating block and are uncorrected. Elemental analysis $(\mathrm{C}, \mathrm{H}, \mathrm{N})$ was performed on a CE440 analyser (Exeter Analytical).

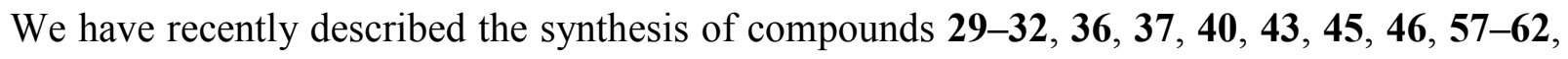
65-72 and 75. ${ }^{25}$ Compounds $18,{ }^{16} \mathbf{3 4},{ }^{32} \mathbf{3 5}^{32}$ and $48^{33}$ have been described by others, and analytical data are in accordance with the published data. 
(1-(4-(Hydroxymethyl)benzyl)piperidin-4-yl)diphenylmethanol (1). Under inert atmosphere, diphenyl(piperidin-4-yl)methanol hydrochloride $\quad(2.1 \quad \mathrm{~g}, \quad 7.0 \mathrm{mmol})$, (4(chloromethyl)phenyl)methanol (1.0 g, $6.4 \mathrm{mmol})$, potassium carbonate $(2.9 \mathrm{~g}, 21.0 \mathrm{mmol})$ and potassium iodide $(0.1 \mathrm{~g}, 0.64 \mathrm{mmol})$ were heated in acetone $(25 \mathrm{ml})$ for $6 \mathrm{~h}$. After cooling the inorganic components were filtered off and the filtrate was purified by column chromatography $($ TEA 0.5\%; DCM: methanol $=100: 0 \rightarrow 98: 2$ ). The resulting colorless oil was triturated with diethyl ether to give a white solid (0.3 g, 13\%). Mp: $119{ }^{\circ} \mathrm{C} .{ }^{1} \mathrm{H}$ NMR (DMSO- $\left.d_{6}, 500 \mathrm{MHz}\right): \delta$ $7.48(\mathrm{~d}, J=7.4 \mathrm{~Hz}, 4 \mathrm{H}, \mathrm{ph}-2,6 H), 7.29-7.22(\mathrm{~m}, 8 \mathrm{H}, \mathrm{ph}-2,3,5,6 H$, ph'-3,5H), $7.10(\mathrm{t}, J=7.3 \mathrm{~Hz}$, $2 \mathrm{H}, \mathrm{ph}-4 H), 5.24(\mathrm{~s}, 1 \mathrm{H}, \mathrm{COH}), 5.11\left(\mathrm{t}, J=4.9 \mathrm{~Hz}, 1 \mathrm{H}, \mathrm{CH}_{2} \mathrm{OH}\right), 4.46(\mathrm{~d}, J=5.5 \mathrm{~Hz}, 2 \mathrm{H}$, $\mathrm{CH}_{2} \mathrm{OH}$ ), 3.41 (br s, 2H, NCH$H_{2}$ ), 2.81 (br s, 2H, pip-2,6H ${ }^{\text {eq }}$ ), 2.53-2.49 (m, 1H, pip-4H), 2.06 (br s, 2H, pip-2,6 $\left.H^{a x}\right), 1.49-1.47\left(\mathrm{~m}, 2 \mathrm{H}\right.$, pip-3,5H $\left.H^{a x}\right), 1.24\left(\mathrm{~d}, J=11.1 \mathrm{~Hz}, 2 \mathrm{H}, \mathrm{pip}-3,5 H^{e q}\right) .{ }^{13} \mathrm{C}$ NMR (DMSO- $d_{6}, 125$ MHz): $\delta 147.3$ (ph'-1C), 128.7 (ph-1C), 127.8 (ph'-2,6C), 126.3 (ph'-4C), 125.8 (ph-4C), 125.7 (ph'-3,5C, ph-2,3,5,6C), $78.5(\mathrm{COH}), 62.7\left(\mathrm{CH}_{2} \mathrm{OH}\right), 62.3\left(\mathrm{NCH}_{2}\right), 53.3$ (pip-2,6C), 43.3 (pip-4C), 25.9 (pip-3,5C). HRMS (m/z): $[\mathrm{M}+\mathrm{H}]^{+}$calcd. for $\mathrm{C}_{26} \mathrm{H}_{29} \mathrm{NO}_{2}$, 386.2120; found, 386.2129. Elemental analysis: calcd. for $\mathrm{C}_{26} \mathrm{H}_{29} \mathrm{NO}_{2} \cdot 0.75 \mathrm{H}_{2} \mathrm{O}, \mathrm{C} 77.87, \mathrm{H}$ 7.67, N 3.49; found, C 77.94, H 7.39, N 3.49.

4-((4-(Hydroxydiphenylmethyl)piperidin-1-yl)methyl)benzaldehyde (2). Oxalyl chloride $(0.22 \mathrm{ml}, 2.6 \mathrm{mmol})$ was dissolved in $\operatorname{DCM}(5 \mathrm{ml})$ and the solution was cooled to $<-50{ }^{\circ} \mathrm{C}$. Under inert conditions, DMSO $(0.22 \mathrm{ml}, 3.1 \mathrm{mmol})$ dissolved in DCM (2 ml) was added dropwise and the solution was stirred for $15 \mathrm{~min}$. Alcohol 1 (0.25 g, $0.65 \mathrm{mmol})$ dissolved in $\operatorname{DCM}(5 \mathrm{ml})$ was added and the solution was stirred for further $15 \mathrm{~min}$. The reaction was quenched with TEA $(0.8 \mathrm{ml}, 5.7 \mathrm{mmol})$ and allowed to warm to room temperature. Water (30 ml) was added and the solution extracted with DCM. The organic phase was dried $\left(\mathrm{MgSO}_{4}\right)$, 
filtered, concentrated and crystallized using a mixture of petroleum ether: ethyl acetate $=3: 2$ to give the product as a white solid (0.09 g, 34\%). Mp: $149{ }^{\circ} \mathrm{C} .{ }^{1} \mathrm{H}$ NMR (DMSO- $\left.d_{6}, 500 \mathrm{MHz}\right): \delta$ 9.98 (s, 1H, CHO), 7.82 (d, $J=8.2 \mathrm{~Hz}, 4 \mathrm{H}, \mathrm{ph}-2,6 H), 7.53-7.48$ (m, 6H, ph-3,5H, ph'-2,6H), $7.23\left(\mathrm{t}, J=7.8 \mathrm{~Hz}, 4 \mathrm{H}, \mathrm{ph}{ }^{\prime}-3,5 H\right), 7.10\left(\mathrm{t}, J=7.3 \mathrm{~Hz}, 2 \mathrm{H}, \mathrm{ph}{ }^{\prime}-4 H\right), 5.23(\mathrm{~s}, 1 \mathrm{H}, \mathrm{COH}), 3.51(\mathrm{~s}$, 2H, $\left.\mathrm{NCH}_{2}\right), 2.76\left(\mathrm{~d}, J=11.2 \mathrm{~Hz}, 2 \mathrm{H}\right.$, pip-2,6H $\left.H^{e q}\right), 2.53-2.45(\mathrm{~m}, 1 \mathrm{H}$, pip- $4 H), 1.97(\mathrm{t}, J=11.0$ $\mathrm{Hz}, 2 \mathrm{H}$, pip-2,6 $\left.H^{a x}\right), 1.53-1.44\left(\mathrm{~m}, 2 \mathrm{H}, \mathrm{pip}-3,5 H^{a x}\right), 1.23\left(\mathrm{~d}, J=12.8 \mathrm{~Hz}, 2 \mathrm{H}, \mathrm{pip}-3,5 H^{e q}\right) .{ }^{13} \mathrm{C}$ NMR (DMSO- $\left.d_{6}, 150 \mathrm{MHz}\right): \delta 192.8$ (CHO), 147.3 (ph'-1C), 146.2 (ph-4C), 135.1 (ph-1C), 129.5 (ph-2,6C), 129.2 (ph-3,5C), 127.8 (ph'-3,5C), 125.8 (ph'-4C), 125.8 (ph'-2,6C), 78.5 $(C O H), 62.0\left(\mathrm{NCH}_{2}\right), 53.6$ (pip-2,6C), 43.3 (pip-4C), 26.0 (pip-3,5C). HRMS (m/z): [M+H $]^{+}$ calcd. for $\mathrm{C}_{26} \mathrm{H}_{27} \mathrm{NO}_{2}, 386.2120$; found, 386.2106. Elemental analysis: calcd. for $\mathrm{C}_{26} \mathrm{H}_{27} \mathrm{NO}_{2} \cdot 0.5$ $\mathrm{H}_{2} \mathrm{O}, \mathrm{C} 79.16, \mathrm{H} 7.15, \mathrm{~N} 3.55$; found, C 78.95, H 6.90, N 3.48.

Methyl 4-((4-(hydroxydiphenylmethyl)piperidin-1-yl)methyl)benzoate (3). Under inert atmosphere, diphenyl(piperidin-4-yl)methanol hydrochloride (2.0 g, $6.6 \mathrm{mmol})$, methyl 4(bromomethyl)benzoate $(1.26 \mathrm{~g}, 5.5 \mathrm{mmol})$, potassium carbonate $(2.73 \mathrm{~g}, 19.8 \mathrm{mmol})$ and potassium iodide were suspended in acetonitrile $(50 \mathrm{ml})$ and refluxed overnight. After cooling to room temperature the inorganic components were filtered off and the filtrate was purified by column chromatography (TEA; DCM: methanol =100: $0 \rightarrow 98: 2)$. The product was obtained as a white solid (1.4 g, 61\%). Mp: $116{ }^{\circ} \mathrm{C} .{ }^{1} \mathrm{H}$ NMR (DMSO- $\left.d_{6}, 300 \mathrm{MHz}\right): \delta 7.87(\mathrm{~d}, J=8.2 \mathrm{~Hz}$, 2H, ph-2,6H), 7.48 (d, $J=7.6 \mathrm{~Hz}, 4 \mathrm{H}, \mathrm{ph} '-2,6 H), 7.41(\mathrm{~d}, J=8.2 \mathrm{~Hz}, 2 \mathrm{H}, \mathrm{ph}-3,5 H), 7.23(\mathrm{t}, J=$ $\left.7.7 \mathrm{~Hz}, 4 \mathrm{H}, \mathrm{ph}{ }^{\prime}-3,5 H\right), 7.09$ (t, $\left.J=7.3,2 \mathrm{H}, \mathrm{ph}{ }^{-4 H}\right), 5.25(\mathrm{~s}, 1 \mathrm{H}, \mathrm{OH}), 3.81\left(\mathrm{~s}, 3 \mathrm{H}, \mathrm{OCH}_{3}\right), 3.47$ $\left(\mathrm{s}, 2 \mathrm{H}, \mathrm{NCH}_{2}\right), 2.74\left(\mathrm{~d}, J=10.8 \mathrm{~Hz}, 2 \mathrm{H}, \mathrm{pip}-2,6 H^{e q}\right), 2.50-2.43(\mathrm{~m}, 1 \mathrm{H}, \mathrm{pip}-4 H), 1.94(\mathrm{t}, J=11.0$ $\mathrm{Hz}, 2 \mathrm{H}$, pip-2,6H $\left.H^{a x}\right), 1.54-1.43\left(\mathrm{~m}, 2 \mathrm{H}, \mathrm{pip}-3,5 H^{a x}\right), 1.23\left(\mathrm{~d}, J=12.1 \mathrm{~Hz}, 2 \mathrm{H}, \mathrm{pip}-3,5 H^{e q}\right) .{ }^{13} \mathrm{C}$ NMR (DMSO- $\left.d_{6}, 75 \mathrm{MHz}\right): \delta 166.1(C=\mathrm{O}), 147.2$ (ph'-1C), 144.6 (ph-4C), 129.0 (ph-2,6C), 
128.7 (ph-3,5C), 128.1 (ph-1C), 127.7 (ph'-3,5C), 125.7 (ph'-4C), 125.7 (ph'-2,6C), 78.4 (COH), $61.8\left(\mathrm{NCH}_{2}\right), 53.5$ (pip-2,6C), $52.0\left(\mathrm{OCH}_{3}\right), 43.3$ (pip-4C), 25.8 (pip-3,5C). HRMS (m/z): $[\mathrm{M}+\mathrm{H}]^{+}$calcd. for $\mathrm{C}_{27} \mathrm{H}_{29} \mathrm{NO}_{3}, 415.2142$; found, 415.2131. Elemental analysis: calcd. for $\mathrm{C}_{27} \mathrm{H}_{29} \mathrm{NO}_{3}, \mathrm{C} 78.04, \mathrm{H} 7.03, \mathrm{~N} 3.37$; found, C 77.79, H 7.04, N 3.32.

4-((4-(Hydroxydiphenylmethyl)piperidin-1-yl)methyl)benzoic acid (4). Compound 3 (1.0 g, $2.4 \mathrm{mmol})$ was dissolved in methanol $(2 \mathrm{ml})$ and THF $(2 \mathrm{ml})$. Aqueous potassium hydroxide (2 $\mathrm{M}, 4 \mathrm{ml}$ ) was added and the mixture was heated in the microwave oven for $15 \mathrm{~min}$ at $70{ }^{\circ} \mathrm{C}$. After cooling the organic solvents were removed under reduced pressure and the aqueous solution neutralized with $\mathrm{HCl}$. The product precipitated as white solid that was recrystallized from 2-propanol under addition of a drop of water $(0.71 \mathrm{~g}, 74 \%)$. Mp: $>250{ }^{\circ} \mathrm{C} .{ }^{1} \mathrm{H}$ NMR (DMSO- $\left.d_{6}, 500 \mathrm{MHz}\right): \delta 7.91(\mathrm{~d}, J=8.1 \mathrm{~Hz}, 2 \mathrm{H}, \mathrm{ph}-2,6 H), 7.53-7.48(\mathrm{~m}, 6 \mathrm{H}, \mathrm{ph}-3,5 H$, ph'2,6H), 7.24 (t, $J=7.7 \mathrm{~Hz}, 4 \mathrm{H}, \mathrm{ph}-3,5 H), 7.11$ (t, $J=7.3 \mathrm{~Hz}, 2 \mathrm{H}, \mathrm{ph}$ '-4H), 5.54 (s, 1H, OH), 3.77

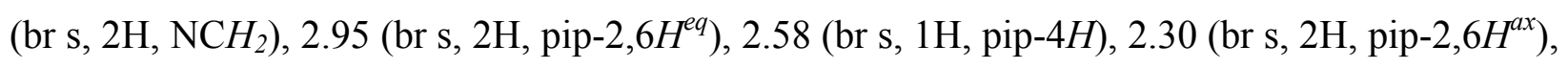
1.63-1.59 (m, 2H, pip-3,5H $\left.H^{a x}\right), 1.28\left(\mathrm{~d}, J=12.6 \mathrm{~Hz}, 2 \mathrm{H}\right.$, pip-3,5 $\left.H^{a x}\right) .{ }^{13} \mathrm{C}$ NMR (DMSO-d 6,125 MHz): $\delta 167.2(C=\mathrm{O}), 147.0$ (ph'-1C), 139.8 (ph-4C), 130.3 (ph-1C), 129.8 (ph-3,5C), 129.3 (ph-2,6C), 127.8 (ph'-3,5C), 125.9 (ph'-4C), 125.7 (ph'-2,6C), $78.4(\mathrm{COH}), 60.5\left(\mathrm{NCH}_{2}\right), 52.7$ (pip-2,6C), 43.4 (pip-4C), 25.0 (pip-3,5C). HRMS (m/z): $[\mathrm{M}+\mathrm{H}]^{+}$calcd. for $\mathrm{C}_{26} \mathrm{H}_{27} \mathrm{NO}_{3}$, 402.2069; found, 402.2085. Elemental analysis: calcd. for $\mathrm{C}_{26} \mathrm{H}_{27} \mathrm{NO}_{3} \cdot \mathrm{H}_{2} \mathrm{O}, \mathrm{C} 74.44, \mathrm{H}$ 6.97, N 3.34; found, C 74.28, H 6.64, N 3.34 .

Methyl 4-(2-bromoacetyl)benzoate (5). Methyl 4-acetylbenzoate $(2 \mathrm{~g}, 0.011 \mathrm{~mol})$ and $p$ toluenesulfonic acid $(0.2 \mathrm{~g}, 1.2 \mathrm{mmol})$ were suspended in anhydrous acetonitrile $(10 \mathrm{ml}) . \mathrm{N}$ Bromosuccinimide (2.2 g, $11.2 \mathrm{mmol})$ was added under inert conditions. The mixture was stirred for $15 \mathrm{~min}$ at room temperature and subsequently heated to $60{ }^{\circ} \mathrm{C}$ for $2 \mathrm{~h}$. After cooling, the 
mixture was stirred in a mixture of water $(50 \mathrm{ml})$ and toluene $(50 \mathrm{ml})$ for $15 \mathrm{~min}$. The organic layer was washed with brine, dried $\left(\mathrm{MgSO}_{4}\right)$ and concentrated to dryness resulting in a light yellow solid, which was again washed with toluene to remove remaining bromine (67-85\%). Mp: $78-80{ }^{\circ} \mathrm{C} .{ }^{1} \mathrm{H}$ NMR (DMSO- $\left.d_{6}, 300 \mathrm{MHz}\right): \delta 8.02(\mathrm{~s}, 4 \mathrm{H}, \mathrm{ph}-2,3,5,6 H), 3.82\left(\mathrm{~s}, 3 \mathrm{H}, \mathrm{OCH}_{3}\right)$, $2.60\left(\mathrm{~s}, 2 \mathrm{H}, \mathrm{CH}_{2}\right) .{ }^{13} \mathrm{C}$ NMR (DMSO- $\left.d_{6}, 75 \mathrm{MHz}\right): \delta 195.8(C=\mathrm{O}$ ketone), $165.6(C=\mathrm{O}$ ester), 137.2 (ph-4C), 133.1 (ph-1C), 129.6 (ph-2,6C), 129.0 (ph-3,5C), $70.9\left(\mathrm{ph}-\mathrm{CH}_{2}\right), 57.7\left(\mathrm{OCH}_{3}\right)$. $\operatorname{HRMS}(\mathrm{m} / \mathrm{z}):[\mathrm{M}+\mathrm{H}]^{+}$calcd. for $\mathrm{C}_{10} \mathrm{H}_{9} \mathrm{BrO}_{3}, 256.9813$; found, 256.9818 .

\section{Methyl 4-(2-(4-(hydroxydiphenylmethyl)piperidin-1-yl)acetyl)benzoate (6). Diphenyl-} piperidin-4-yl-methanol hydrochloride (1.3 g, $4.3 \mathrm{mmol})$, compound 5 (1.0 g, $3.9 \mathrm{mmol})$ and potassium carbonate $(1.2 \mathrm{~g}, 8.7 \mathrm{mmol})$ were stirred in acetone $(30 \mathrm{ml})$ at room temperature. After $12 \mathrm{~h}$ the inorganic salts were filtered off and the filtrate concentrated under reduced pressure. The product was purified by column chromatography (TEA $0.5 \%$; DCM: methanol $=100: 0 \rightarrow$ 98: 2). Re-crystallization from acetonitrile gave a yellow solid $(0.5 \mathrm{~g}, 30 \%)$. Mp: $140{ }^{\circ} \mathrm{C} .{ }^{1} \mathrm{H}$ NMR (DMSO- $d_{6}, 500$ MHz): $\delta 8.08-8.03(\mathrm{~m}, 4 \mathrm{H}, \mathrm{ph}-2,3,5,6 H), 7.48(\mathrm{~d}, J=8.1 \mathrm{~Hz}, 4 \mathrm{H}, \mathrm{ph}-$ 2,6H), $7.24(\mathrm{t}, J=7.8 \mathrm{~Hz}, 4 \mathrm{H}, \mathrm{ph}-3,5 H), 7.10\left(\mathrm{t}, J=7.3 \mathrm{~Hz}, 2 \mathrm{H}, \mathrm{ph}{ }^{\prime}-4 H\right), 5.23(\mathrm{~s}, 1 \mathrm{H}, \mathrm{OH}), 3.87$ (s, 3H, $\mathrm{OCH}_{3}$ ), $3.77\left(\mathrm{~s}, 2 \mathrm{H}, \mathrm{NCH}_{2}\right), 2.84\left(\mathrm{~d}, J=10.7 \mathrm{~Hz}, 2 \mathrm{H}\right.$, pip-2,6H ${ }^{e q}$ ), 2.49 (overlay with DMSO peak, pip-4H), $2.10\left(\mathrm{t}, J=11.1 \mathrm{~Hz}, 2 \mathrm{H}\right.$, pip-2,6 $\left.H^{a x}\right), 1.50-1.43\left(\mathrm{~m}, 2 \mathrm{H}, \mathrm{pip}-3,5 H^{a x}\right), 1.21$ $\left(\mathrm{d}, J=12.3 \mathrm{~Hz}, 2 \mathrm{H}, \mathrm{pip}-3,5 H^{e q}\right) .{ }^{13} \mathrm{C}$ NMR (DMSO- $\left.d_{6}, 125 \mathrm{MHz}\right): \delta 197.2(C=\mathrm{O}$ ketone), 165.6 $(C=$ O ester), 147.3 (ph'-1C), 139.4 (ph-4C), 133.1 (ph-1C), 129.3 (ph-2,6C), 128.5 (ph-3,5C), 127.8 (ph'-3,5C), 125.7 (ph'-2,4,6C), $78.5(\mathrm{COH}), 64.6\left(\mathrm{NCH}_{2}\right), 53.7$ (pip-2,6C), $52.5\left(\mathrm{OCH}_{3}\right)$, 43.0 (pip-4C), 25.9 (pip-3,5C). HRMS (m/z): $[\mathrm{M}+\mathrm{H}]^{+}$calcd. for $\mathrm{C}_{28} \mathrm{H}_{29} \mathrm{NO}_{4}, 444.2175$; found, 444.2172. 
4-(2-(4-(Hydroxydiphenylmethyl)piperidin-1-yl)acetyl)benzoic acid (7). Compound 6 (0.3 $\mathrm{g}, 0.7 \mathrm{mmol})$ was dissolved in methanol $(1 \mathrm{ml})$ and THF $(1 \mathrm{ml})$. Aqueous potassium hydroxide ( $2 \mathrm{M}, 2 \mathrm{ml}$ ) was added and the mixture was heated in the microwave oven for $15 \mathrm{~min}$ at $70{ }^{\circ} \mathrm{C}$. After cooling the organic solvents were removed under reduced pressure and the aqueous solution neutralized with $\mathrm{HCl}$. The product precipitated as an off-white solid that was recrystallized from acetonitrile under addition of a drop of water $(0.1 \mathrm{~g}, 35 \%)$. Mp: $187{ }^{\circ} \mathrm{C} .{ }^{1} \mathrm{H}$ NMR (DMSO-d 6,500 MHz): $\delta 8.04-8.00(\mathrm{~m}, 4 \mathrm{H}, \mathrm{ph}-2,3,5,6 H), 7.48(\mathrm{~d}, J=8.0 \mathrm{~Hz}, 4 \mathrm{H}, \mathrm{ph}$ 2,6H), $7.24(\mathrm{t}, J=7.7 \mathrm{~Hz}, 4 \mathrm{H}, \mathrm{ph}-3,5 H), 7.10$ (t, $\left.J=7.2 \mathrm{~Hz}, 2 \mathrm{H}, \mathrm{ph}{ }^{\prime}-4 H\right), 5.24(\mathrm{~s}, 1 \mathrm{H}, \mathrm{OH}), 3.80$ (s, 2H, $\mathrm{NCH}_{2}$ ), 2.86 (d, $J=9.2 \mathrm{~Hz}, 2 \mathrm{H}$, pip-2,6H ${ }^{e q}$ ), 2.49 (overlay with DMSO peak, pip-4H), 2.14-2.10 (m, 2H, pip-2,6 $\left.H^{a x}\right), 1.52-1.47\left(\mathrm{~m}, 2 \mathrm{H}, \mathrm{pip}-3,5 H^{a x}\right), 1.22\left(\mathrm{~d}, J=11.6,2 \mathrm{H}, \mathrm{pip}-3,5 H^{\mathrm{eq}}\right)$. ${ }^{13} \mathrm{C}$ NMR (DMSO- $\left.d_{6}, 125 \mathrm{MHz}\right): \delta 197.0$ ( $C=\mathrm{O}$ ketone), 166.7 ( $C=\mathrm{O}$ ester), 147.2 (ph'-1C), 138.8 (ph-4C), 135.1 (ph-1C), 129.4 (ph-3,5C), 128.3 (ph-2,6C), 127.8 (ph'-3,5C), 125.8 (ph'4C), 125.7 (ph'-2,6C), $78.5(\mathrm{COH}), 64.4\left(\mathrm{NCH}_{2}\right), 53.6$ (pip-2,6C), 43.9 (pip-4C), 25.8 (pip3,5C). HRMS (m/z): $[\mathrm{M}-\mathrm{H}]^{+}$calcd. for $\mathrm{C}_{27} \mathrm{H}_{27} \mathrm{NO}_{4}$, 428.1862; found, 428.1843. Elemental analysis: calcd. for $\mathrm{C}_{27} \mathrm{H}_{27} \mathrm{NO}_{4} \cdot 0.5 \mathrm{H}_{2} \mathrm{O}, \mathrm{C} 73.95, \mathrm{H} 6.44, \mathrm{~N} 3.19$; found, C 74.10, H 6.23, N 3.29 .

Methyl 2-(4-(bromomethyl)phenyl)-2-methylpropanoate (8). Under inert atmosphere, $p$ tolylacetic acid $(5 \mathrm{~g}, 0.033 \mathrm{~mol})$ was dissolved in methanol $(60 \mathrm{ml})$ and under cooling (ice bath) chlorotrimethyl silane $(6.5 \mathrm{ml})$ was added dropwise. The solution was stirred overnight at room temperature and subsequently concentrated to dryness. The resulting colorless oil was dissolved in THF $(25 \mathrm{ml})$ and added to a solution of sodium hydride (4 g, 60\% dispersion in mineral oil, $0.1 \mathrm{~mol})$ in THF $(100 \mathrm{ml})$. Methyl iodide $(5 \mathrm{ml}, 0.08 \mathrm{~mol})$ was added dropwise and under cooling. The resulting suspension was stirred overnight at room temperature. After concentration 
to dryness the crude was taken up in water $(50 \mathrm{ml})$ and extracted with ethyl acetate $(3 \times 50 \mathrm{ml})$. The organic layer was dried $\left(\mathrm{MgSO}_{4}\right)$ and concentrated to give methyl 2-methyl-2- $(p$ tolyl)propanoate (8a) as a yellow oil $(4.6 \mathrm{~g} ; 73 \%) .{ }^{1} \mathrm{H}$ NMR $\left(\mathrm{CDCl}_{3}, 300 \mathrm{MHz}\right): \delta 7.32(\mathrm{~d}, J=$ $8.3 \mathrm{~Hz}, 2 \mathrm{H}, \mathrm{ph}-2,6 H), 7.17$ (d, $J=8.1 \mathrm{~Hz}, 2 \mathrm{H}, \mathrm{ph}-3,5 H), 3.66$ (s, 3H, OCH $), 2.34$ (s, 3H, ph$\left.\mathrm{CH}_{3}\right), 1.58\left(\mathrm{~s}, 6 \mathrm{H}, \mathrm{C}\left(\mathrm{CH}_{3}\right)_{2}\right) \cdot{ }^{13} \mathrm{C} \mathrm{NMR}\left(\mathrm{CDCl}_{3}, 75 \mathrm{MHz}\right): \delta 177.4(\mathrm{C}=\mathrm{O}), 141.8(\mathrm{ph}-4 \mathrm{C}), 136.3$ (ph-1C), 129.1 (ph-3,5C), $\left.125.5(\mathrm{ph}-2,6 C), 52.2\left(\mathrm{OCH}_{3}\right), 46.2\left(\mathrm{C}\left(\mathrm{CH}_{3}\right)_{2}\right), 26.6\left(\mathrm{C}_{(\mathrm{CH}}\right)_{2}\right), 21.0$ (ph- $\left.\mathrm{CH}_{3}\right)$. HRMS (m/z): $[\mathrm{M}+\mathrm{H}]^{+}$calcd. for $\mathrm{C}_{12} \mathrm{H}_{16} \mathrm{O}_{2}$, 193.1229; found, 193.1236. A solution of 2,2'-azobis(2-methylpropionitrile) in toluene $(5 \mathrm{ml}, 0.2 \mathrm{M})$ was concentrated to dryness without heating and under exclusion of light. 2,2'-Azobis(2-methylpropionitrile), $N$-bromosuccinimide $(1.0 \mathrm{~g}, 5 \mathrm{mmol})$ and compound $8 \mathrm{a}(1.0 \mathrm{~g}, 5 \mathrm{mmol})$ were dissolved in bromobenzene or chlorobenzene $(5 \mathrm{ml})$. The solution was heated in an oil bath kept at $110{ }^{\circ} \mathrm{C}$. When the reaction mixture reached $80-90{ }^{\circ} \mathrm{C}$, an exothermic reaction started with the development of bromine (orange color). Subsequently, the mixture turned dark brown and was quenched by cooling with an ice bath and adding ice water. The product was extracted with petrol. The organic layer was washed with brine, dried $\left(\mathrm{MgSO}_{4}\right)$ and concentrated to dryness. The product was purified by column chromatography (petrol: ethyl acetate $=100: 0 \rightarrow 98: 2)$ giving a yellow oil $(50-61 \%)$. ${ }^{1} \mathrm{H}$ NMR (DMSO-d, $\left.300 \mathrm{MHz}\right): \delta 7.38(\mathrm{~d}, J=8.3 \mathrm{~Hz}, 2 \mathrm{H}, \mathrm{ph}-2,6 H), 7.29(\mathrm{~d}, J=8.4 \mathrm{~Hz}, 2 \mathrm{H}$, ph-3,5H), $4.67\left(\mathrm{~s}, 2 \mathrm{H}, \mathrm{CH}_{2} \mathrm{Br}\right), 3.57\left(\mathrm{~s}, 3 \mathrm{H}, \mathrm{OCH}_{3}\right), 1.48\left(\mathrm{~s}, 6 \mathrm{H}, \mathrm{C}\left(\mathrm{CH}_{3}\right)_{2}\right) .{ }^{13} \mathrm{C} \mathrm{NMR}\left(\mathrm{DMSO}-d_{6}\right.$, $125 \mathrm{MHz}): \delta 176.4(C=\mathrm{O}), 142.9(\mathrm{ph}-4 C), 140.9(\mathrm{ph}-1 C), 126.6(\mathrm{ph}-2,6 C), 125.1(\mathrm{ph}-3,5 C), 62.5$ $\left(\mathrm{CH}_{2} \mathrm{Br}\right), 52.0\left(\mathrm{OCH}_{3}\right), 46.0\left(\mathrm{C}\left(\mathrm{CH}_{3}\right)_{2}\right), 26.4\left(\mathrm{C}\left(\mathrm{CH}_{3}\right)_{2}\right)$. HRMS (m/z): [M-H] $]^{+}$calcd. for $\mathrm{C}_{12} \mathrm{H}_{15} \mathrm{BrO}_{2}, 269.0172$; found, 269.0163.

Methyl 2-(4-((4-(hydroxydiphenylmethyl)piperidin-1-yl)methyl)phenyl)-2-methyl-

propanoate (9). Diphenyl(piperidin-4-yl)methanol hydrochloride (1.0 g, $3.3 \mathrm{mmol})$, compound 
$8(0.7 \mathrm{~g}, 2.6 \mathrm{mmol})$, potassium carbonate $(1.46 \mathrm{~g}, 10.6 \mathrm{mmol})$ and potassium iodide $(0.05 \mathrm{~g}, 0.33$ mmol) were suspended in acetonitrile $(25 \mathrm{ml})$. Under inert atmosphere the mixture was refluxed overnight. After cooling the inorganic components were removed by filtration. The filtrate was concentrated to dryness and the crude purified by column chromatography (TEA 0.5\%; DCM: methanol $=100: 0 \rightarrow 98: 2)$. The product was isolated as colorless oil. ${ }^{1} \mathrm{H}$ NMR $\left(\mathrm{DMSO}-d_{6}, 500\right.$ MHz): $\delta 7.48(\mathrm{~d}, J=7.6 \mathrm{~Hz}, 4 \mathrm{H}, \mathrm{ph}-2,6 H), 7.29-7.18(\mathrm{~m}, 8 \mathrm{H}, \mathrm{ph}-2,3,5,6 H, \mathrm{ph}$ '-3,5H), $7.10(\mathrm{t}, J$ $\left.=7.1 \mathrm{~Hz}, 2 \mathrm{H}, \mathrm{ph}{ }^{\prime}-4 H\right), 5.22(\mathrm{~s}, 1 \mathrm{H}, \mathrm{OH}), 3.56\left(\mathrm{~s}, 3 \mathrm{H}, \mathrm{OCH}_{3}\right), 3.37\left(\mathrm{~s}, 2 \mathrm{H}, \mathrm{NCH}_{2}\right), 2.75(\mathrm{~d}, J=$ $10.8 \mathrm{~Hz}, 2 \mathrm{H}$, pip-2,6 $\left.H^{e q}\right), 2.49-2.44(\mathrm{~m}, 1 \mathrm{H}, \mathrm{pip}-4 H), 1.91\left(\mathrm{t}, J=11.3 \mathrm{~Hz}, 2 \mathrm{H}, \mathrm{pip}-2,6 H^{a x}\right), 1.50-$ $1.43\left(\mathrm{~m}, 8 \mathrm{H}\right.$, pip-3,5H $\left.H^{a x}, \mathrm{C}\left(\mathrm{CH}_{3}\right)_{2}\right), 1.23\left(\mathrm{~d}, J=12.2 \mathrm{~Hz}, 2 \mathrm{H}, \mathrm{pip}-3,5 H^{e q}\right) .{ }^{13} \mathrm{C}$ NMR (DMSO- $d_{6}$, $125 \mathrm{MHz}): \delta 167.4(C=\mathrm{O}), 147.3$ (ph'-1C), 143.0 (ph-1C), 137.0 (ph-4C), 128.7 (ph-3,5C), 127.7 (ph'-3,5C), 125.8 (ph'-2,6C, ph-2,6C), 125.2 (ph'-4C), $78.5(C \mathrm{OH}), 61.9\left(\mathrm{NCH}_{2}\right), 53.5$

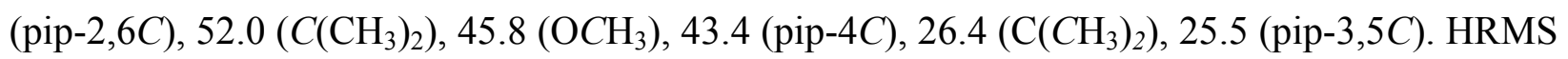
$(\mathrm{m} / \mathrm{z}):[\mathrm{M}+\mathrm{H}]^{+}$calcd. for $\mathrm{C}_{30} \mathrm{H}_{35} \mathrm{NO}_{3}, 458.2695$; found, 458.2696.

\section{2-(4-((4-(Hydroxydiphenylmethyl)piperidin-1-yl)methyl)phenyl)-2-methylpropanoic acid}

(10). Compound 9 (0.5 g, $1.0 \mathrm{mmol})$ was dissolved in methanol $(1 \mathrm{ml})$ and THF $(1 \mathrm{ml})$. Aqueous potassium hydroxide $(2 \mathrm{M}, 2 \mathrm{ml})$ was added and the mixture was heated in the microwave oven for $15 \mathrm{~min}$ at $70^{\circ} \mathrm{C}$. After cooling the organic solvents were evaporated and the aqueous solution neutralized with $\mathrm{HCl}$. The product precipitated as white solid that was recrystallized from acetonitrile under addition of a drop of water $(0.23 \mathrm{~g}, 48 \%)$. Mp: $246{ }^{\circ} \mathrm{C} .{ }^{1} \mathrm{H}$ NMR (DMSO- $d_{6}$, $500 \mathrm{MHz}): \delta 7.48\left(\mathrm{~d}, J=7.7 \mathrm{~Hz}, 4 \mathrm{H}, \mathrm{ph}{ }^{\prime}-2,6 H\right), 7.30-7.19(\mathrm{~m}, 8 \mathrm{H}, \mathrm{ph}-2,3,5,6 H, \mathrm{ph}-3,5 H), 7.09$ (t, $J=7.3 \mathrm{~Hz}, 2 \mathrm{H}, \mathrm{ph}$ '-4H), $5.22(\mathrm{~s}, 1 \mathrm{H}, \mathrm{OH}), 3.39\left(\mathrm{~s}, 2 \mathrm{H}, \mathrm{NCH}_{2}\right), 2.77$ (d, $J=10.6 \mathrm{~Hz}, 2 \mathrm{H}$, pip2,6H $\left.H^{e q}\right), 2.49-2.44\left(\mathrm{~m}, 1 \mathrm{H}\right.$, pip-4H), $1.94\left(\mathrm{t}, J=11.2 \mathrm{~Hz}, 2 \mathrm{H}, \mathrm{pip}-2,6 H^{a x}\right), 1.50-1.43$ (m, 8H, pip$\left.3,5 H^{a x}, \mathrm{C}\left(\mathrm{CH}_{3}\right)_{2}\right), 1.20\left(\mathrm{~d}, J=12.4 \mathrm{~Hz}, 2 \mathrm{H}, \mathrm{pip}-3,5 H^{e q}\right) .{ }^{13} \mathrm{C}$ NMR (DMSO- $\left.d_{6}, 125 \mathrm{MHz}\right): \delta$ 
$177.6(C=\mathrm{O}), 147.3$ (ph'-1C), $143.6(\mathrm{ph}-4 C), 136.2(\mathrm{ph}-1 C), 128.6(\mathrm{ph}-2,6 C), 127.7$ (ph'-3,5C), 125.8 (ph'-2,6C, ph-3,5C), 125.3 (ph'-4C), $78.5(\mathrm{COH}), 61.9\left(\mathrm{NCH}_{2}\right), 53.5$ (pip-2,6C), 45.5

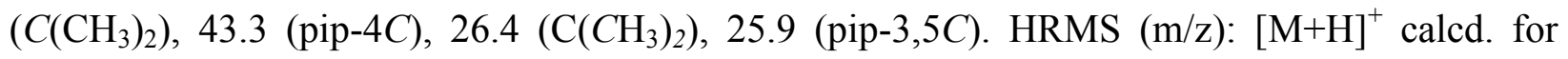
$\mathrm{C}_{29} \mathrm{H}_{33} \mathrm{NO}_{3}$, 444.2539; found, 444.2520. Elemental analysis: calcd. for $\mathrm{C}_{29} \mathrm{H}_{33} \mathrm{NO}_{3} \cdot 0.5 \mathrm{H}_{2} \mathrm{O}, \mathrm{C}$ 76.96, H 7.57, N 3.09; found, C 77.19, H 7.38, N 3.09.

Methyl 4-(bromomethyl)-3-fluorobenzoate (11). 3-Fluoro-4-methylbenzoic acid (2.0 g, 13.0 mmol) was dissolved in methanol $(20 \mathrm{ml})$. Chlorotrimethyl silane $(2.6 \mathrm{ml})$ was added dropwise and under cooling and the mixture was stirred overnight at room temperature. The resulting crude was concentrated on silica and the product was purified by column chromatography (DCM). Methyl 3-fluoro-4-methylbenzoate (11a) was isolated as a colorless oil $(1.4 \mathrm{~g}, 64 \%) .{ }^{1} \mathrm{H}$ $\operatorname{NMR}\left(\mathrm{CDCl}_{3}, 500 \mathrm{MHz}\right): \delta 7.70(\mathrm{~d}, J=7.9 \mathrm{~Hz}, 1 \mathrm{H}, \mathrm{ph}-6 H), 7.64(\mathrm{~d}, J=10.2 \mathrm{~Hz}, 1 \mathrm{H}, \mathrm{ph}-2 H)$, $7.23(\mathrm{t}, J=7.7 \mathrm{~Hz}, 1 \mathrm{H}, \mathrm{ph}-5 H), 3.89\left(\mathrm{~s}, 3 \mathrm{H}, \mathrm{OCH}_{3}\right), 2.29\left(\mathrm{~s}, 3 \mathrm{H}, \mathrm{ph}-\mathrm{CH}_{3}\right) .{ }^{13} \mathrm{C} \mathrm{NMR}\left(\mathrm{CDCl}_{3}\right.$, $125 \mathrm{MHz}): \delta 166.2(C=\mathrm{O}), 162.2\left(\mathrm{~d},{ }^{1} J_{\mathrm{C}, \mathrm{F}}=118.8 \mathrm{~Hz}, \mathrm{ph}-3 C\right), 131.4\left(\mathrm{~d},{ }^{3} J_{\mathrm{C}, \mathrm{F}}=5.0, \mathrm{ph}-5 C\right)$, $130.6\left(\mathrm{~d},{ }^{2} J_{\mathrm{C}, \mathrm{F}}=17.3 \mathrm{~Hz}, \mathrm{ph}-4 C\right), 129.7\left(\mathrm{~d},{ }^{3} J_{\mathrm{C}, \mathrm{F}}=7.5 \mathrm{~Hz}, \mathrm{ph}-1 C\right), 125.1\left(\mathrm{~d},{ }^{4} J_{\mathrm{C}, \mathrm{F}}=3.6 \mathrm{~Hz}, \mathrm{ph}-\right.$ $6 C), 116.1\left(\mathrm{~d},{ }^{2} J_{\mathrm{C}, \mathrm{F}}=23.9 \mathrm{~Hz}, \mathrm{ph}-2 C\right), 52.2\left(\mathrm{OCH}_{3}\right), 14.9\left(\mathrm{~d},{ }^{3} J_{\mathrm{C}, \mathrm{F}}=3.5 \mathrm{~Hz}, \mathrm{ph}-\mathrm{CH}_{3}\right) .{ }^{19} \mathrm{~F}$ NMR $\left(\mathrm{CDCl}_{3}, 282 \mathrm{MHz}\right): \delta-117.16(\mathrm{ph}-F)$. HRMS (m/z): $[\mathrm{M}+\mathrm{H}]^{+}$calcd. for $\mathrm{C}_{9} \mathrm{H}_{9} \mathrm{FO}_{2}, 169.0665$; found, 169.0662. A solution of 2,2'-azobis(2-methylpropionitrile) in toluene (10 ml, $0.2 \mathrm{M})$ was concentrated to dryness without heating and under exclusion of light. 2,2'-Azobis(2methylpropionitrile), $N$-bromosuccinimide (1.9 g, $10.0 \mathrm{mmol})$ and compound $11 \mathrm{a}$ (1.4 g, 8.3 mmol) were dissolved in chlorobenzene and treated as described for compound $\mathbf{8}$, giving the desired product as yellow oil (1.5 g, quantitative conversion). ${ }^{1} \mathrm{H}$ NMR $\left(\mathrm{CDCl}_{3}, 500 \mathrm{MHz}\right): \delta$ $7.80(\mathrm{~d}, J=8.0 \mathrm{~Hz}, 1 \mathrm{H}, \mathrm{ph}-6 H), 7.71(\mathrm{~d}, J=10.1 \mathrm{~Hz}, 1 \mathrm{H}, \mathrm{ph}-2 H), 7.46(\mathrm{t}, J=7.7 \mathrm{~Hz}, 1 \mathrm{H}, \mathrm{ph}-$ $5 H), 4.51\left(\mathrm{~s}, 2 \mathrm{H}, \mathrm{CH}_{2} \mathrm{Br}\right), 3.92\left(\mathrm{~s}, 3 \mathrm{H}, \mathrm{CH}_{3}\right) .{ }^{13} \mathrm{C} \mathrm{NMR}\left(\mathrm{CDCl}_{3}, 125 \mathrm{MHz}\right): \delta 165.6(\mathrm{C}=\mathrm{O}), 160.3$ 
$\left(\mathrm{d},{ }^{1} J_{\mathrm{C}, \mathrm{F}}=249.3\right.$, ph-3C), $132.5\left(\mathrm{~d},{ }^{3} J_{\mathrm{C}, \mathrm{F}}=7.9, \mathrm{ph}-5 \mathrm{C}\right), 131.4\left(\mathrm{~d},{ }^{2} J_{\mathrm{C}, \mathrm{F}}=22.0, \mathrm{ph}-4 C\right), 130.1(\mathrm{ph}-$ $1 C), 125.7$ (ph-6C), $117.0\left(\mathrm{~d},{ }^{2} J_{\mathrm{C}, \mathrm{F}}=23.1, \mathrm{ph}-2 \mathrm{C}\right), 52.6\left(\mathrm{OCH}_{3}\right), 24.6\left(\mathrm{CH}_{2} \mathrm{Br}\right)$.

Methyl 3-fluoro-4-((4-(hydroxydiphenylmethyl)piperidin-1-yl)methyl)benzoate hydrochloride (12). Under inert atmosphere, diphenyl(piperidin-4-yl)methanol hydrochloride (2.0 g, $6.6 \mathrm{mmol})$, compound $11(1.5 \mathrm{~g}, 6.0 \mathrm{mmol})$ and potassium carbonate $(2.7 \mathrm{~g}, 19.6 \mathrm{mmol})$ were suspended in acetonitrile $(50 \mathrm{ml})$ and refluxed overnight. After cooling, the inorganic components were filtered off and the filtrate concentrated under reduced pressure. The product was purified by column chromatography (TEA $0.5 \%$; DCM: methanol $=100: 0 \rightarrow 98: 2$ ). The oily product was crystallized with $\mathrm{HCl}$ in ethanol to give a white solid (1.7 g, 61\%). Mp: $151-$ $154{ }^{\circ} \mathrm{C} .{ }^{1} \mathrm{H}$ NMR (DMSO- $\left.d_{6}, 500 \mathrm{MHz}\right): \delta 10.72\left(\mathrm{br} \mathrm{s}, 1 \mathrm{H}, \mathrm{N} H^{+}\right), 7.91-7.84(\mathrm{~m}, 2 \mathrm{H}, \mathrm{ph}-5,6 H)$, $7.78(\mathrm{~d}, J=10.0,1 \mathrm{H}, \mathrm{ph}-2 H), 7.45(\mathrm{~d}, J=7.5 \mathrm{~Hz}, 4 \mathrm{H}, \mathrm{ph}-2,6 H), 7.26\left(\mathrm{t}, J=7.7 \mathrm{~Hz}, 4 \mathrm{H}, \mathrm{ph}{ }^{\prime}-\right.$ 3,5H), $7.13(\mathrm{t}, J=7.3 \mathrm{~Hz}, 2 \mathrm{H}, \mathrm{ph}-4 H), 5.54(\mathrm{~s}, 1 \mathrm{H}, \mathrm{OH}), 4.32\left(\mathrm{~s}, 2 \mathrm{H}, \mathrm{NCH}_{2}\right), 3.88(\mathrm{~s}, 3 \mathrm{H}$, $\left.\mathrm{OCH}_{3}\right), 3.36\left(\mathrm{~d}, J=11.1 \mathrm{~Hz}, 2 \mathrm{H}, \mathrm{pip}-2,6 H^{e q}\right), 3.02-2.95\left(\mathrm{~m}, 2 \mathrm{H}, \mathrm{pip}-2,6 H^{a x}\right), 2.81$ (t, $J=11.7$ $\mathrm{Hz}, 1 \mathrm{H}$, pip- $4 H), 1.83-1.76$ (m, 2H, pip-3,5 $\left.H^{a x}\right), 1.42\left(\mathrm{~d}, J=13.7 \mathrm{~Hz}, 2 \mathrm{H}\right.$, pip-3,5H $\left.H^{e q}\right) .{ }^{13} \mathrm{C} \mathrm{NMR}$ (DMSO- $\left.d_{6}, 125 \mathrm{MHz}\right): \delta 164.8(C=\mathrm{O}), 161.0\left(\mathrm{~d},{ }^{1} J_{\mathrm{C}, \mathrm{F}}=219.8 \mathrm{~Hz}, \mathrm{ph}-3 C\right), 164.5$ (ph'-1C), 134.9 (ph-5C), 133.1 (ph-4C), 127.9 (ph`-3,5C), 126.1 (ph’-4C), 125.7 (ph'-2,6C), 125.2 (ph-6C), $121.6(\mathrm{ph}-1 \mathrm{C}), 116.2\left(\mathrm{~d},{ }^{2} J_{\mathrm{C}, \mathrm{F}}=23.8 \mathrm{~Hz}, \mathrm{ph}-2 \mathrm{C}\right), 78.1(\mathrm{COH}), 56.0\left(\mathrm{NCH}_{2}\right), 52.7\left(\mathrm{OCH}_{3}\right), 51.4$ (pip-2,6C), 40.4 (pip-4C), 23.8 (pip-3,5C). ${ }^{19} \mathrm{~F}$ NMR (DMSO- $d_{6}, 282 \mathrm{MHz}$ ): $\delta-113.23($ ph-F). HRMS (m/z): $[\mathrm{M}+\mathrm{H}]^{+}$calcd. for $\mathrm{C}_{27} \mathrm{H}_{28} \mathrm{FNO}_{3}, 434.2131$; found, 434.2131 .

\section{3-Fluoro-4-((4-(hydroxydiphenylmethyl)piperidin-1-yl)methyl)benzoic acid}

(13).

Compound $12(0.5 \mathrm{~g}, 1.0 \mathrm{mmol})$ was dissolved in methanol $(1 \mathrm{ml})$ and THF $(1 \mathrm{ml})$ and treated with aqueous potassium hydroxide $(2 \mathrm{M}, 2 \mathrm{ml})$. The mixture was heated in the microwave oven for $15 \mathrm{~min}$ at $70{ }^{\circ} \mathrm{C}$. After cooling the organic solvents were removed under reduced pressure 
and the aqueous solution neutralized with $\mathrm{HCl}$. The product precipitated as white solid that was recrystallized from acetonitrile under addition of a drop of water $(0.15 \mathrm{~g}, 33 \%)$. Mp: $247{ }^{\circ} \mathrm{C} .{ }^{1} \mathrm{H}$ NMR (DMSO- $\left.d_{6}, 500 \mathrm{MHz}\right): \delta 7.72(\mathrm{~d}, J=7.9 \mathrm{~Hz}, 1 \mathrm{H}, \mathrm{ph}-6 H), 7.58(\mathrm{~d}, J=10.4 \mathrm{~Hz}, 1 \mathrm{H}, \mathrm{ph}-$ 2H), 7.50-7.48 (m, 5H, ph-5H, ph'-2,6H), 7.23 (t, $J=7.8 \mathrm{~Hz}, 4 \mathrm{H}, \mathrm{ph}-3,5 H), 7.10$ (t, $J=7.3 \mathrm{~Hz}$, 2H, ph'-4H), 5.24 (s, 1H, OH), 3.55 (s, 2H, NCH $), 2.79$ (d, $\left.J=11.0 \mathrm{~Hz}, 2 \mathrm{H}, \mathrm{pip}-2,6 H^{e q}\right), 2.50-$ $2.45(\mathrm{~m}, 1 \mathrm{H}, \mathrm{pip}-4 H), 2.02\left(\mathrm{t}, J=11.1 \mathrm{~Hz}, 2 \mathrm{H}, \mathrm{pip}-2,6 H^{a x}\right), 1.52-1.44\left(\mathrm{~m}, 2 \mathrm{H}, \mathrm{pip}-3,5 H^{a x}\right), 1.23$ $\left(\mathrm{d}, J=12.6 \mathrm{~Hz}, 2 \mathrm{H}, \mathrm{pip}-3,5 H^{e q}\right) .{ }^{13} \mathrm{C} \mathrm{NMR}\left(\mathrm{DMSO}-d_{6}, 150 \mathrm{MHz}\right): \delta 166.3\left(\mathrm{~d},{ }^{4} J_{\mathrm{C}, \mathrm{F}}=2.3 \mathrm{~Hz}\right.$, $C=\mathrm{O}), 160.4\left(\mathrm{~d},{ }^{1} J_{\mathrm{C}, \mathrm{F}}=243.9 \mathrm{~Hz}, \mathrm{ph}-3 C\right), 147.3(\mathrm{ph}-1 C), 132.2\left(\mathrm{~d},{ }^{3} J_{\mathrm{C}, \mathrm{F}}=7.2 \mathrm{~Hz}, \mathrm{ph}-5 C\right), 131.6$ $\left(\mathrm{d},{ }^{3} J_{\mathrm{C}, \mathrm{F}}=4.4 \mathrm{~Hz}, \mathrm{ph}-1 C\right), 129.8\left(\mathrm{~d},{ }^{2} J_{\mathrm{C}, \mathrm{F}}=13.1 \mathrm{~Hz}, \mathrm{ph}-4 C\right), 127.8\left(\mathrm{ph}^{\mathrm{6}}-3,5 C\right), 125.8$ (ph'-4C), 125.7 (ph'-2,6C), $125.1\left(\mathrm{~d},{ }^{4} J_{\mathrm{C}, \mathrm{F}}=2.6 \mathrm{~Hz}, \mathrm{ph}-6 C\right), 115.7\left(\mathrm{~d},{ }^{2} J_{\mathrm{C}, \mathrm{F}}=23.6 \mathrm{~Hz}, \mathrm{ph}-2 C\right), 78.5$ $(C \mathrm{OH}), 54.5\left(\mathrm{NCH}_{2}\right), 53.3$ (pip-2,6C), 43.1 (pip-4C), 25.9 (pip-3,5C). ${ }^{19} \mathrm{~F}$ NMR (DMSO-d 6,282 $\mathrm{MHz}): \delta-117.833(\mathrm{ph}-F)$. HRMS (m/z): $[\mathrm{M}+\mathrm{H}]^{+}$calcd. for $\mathrm{C}_{26} \mathrm{H}_{26} \mathrm{FNO}_{3}, 420.1975$; found, 420.1961. Elemental analysis: calcd. for $\mathrm{C}_{26} \mathrm{H}_{26} \mathrm{FNO}_{3} \cdot 0.5 \mathrm{H}_{2} \mathrm{O}, \mathrm{C} 79.16, \mathrm{H}$ 7.15, N 3.55; found, C 78.95, H 6.90, N 3.48.

\section{Methyl 4-((4-((4-fluorophenyl)(hydroxy)(phenyl)methyl)piperidin-1-yl)methyl)benzoate}

(14). Under inert atmosphere, compound $34 \quad(0.4 \mathrm{~g}, 1.24 \mathrm{mmol})$, methyl 4(bromomethyl)benzoate $(0.43 \mathrm{~g}, 1.86 \mathrm{mmol})$ and potassium carbonate $(0.43 \mathrm{~g}, 3.1 \mathrm{mmol})$ were suspended in acetone $(15 \mathrm{ml})$ and stirred for $2 \mathrm{~h}$ at room temperature. The inorganic components were subsequently filtered off and the filtrate was concentrated under reduced pressure. Purification by column chromatography (TEA 0.5\%; DCM: methanol $=100: 0 \rightarrow 99: 1$ ) gave the product as a colorless oil $(0.19 \mathrm{~g}, 35 \%) .{ }^{1} \mathrm{H}$ NMR (DMSO- $\left.d_{6}, 600 \mathrm{MHz}\right): \delta 7.87(\mathrm{~d}, J=8.2 \mathrm{~Hz}$, 2H, ph-2,6H), 7.53-7.48 (m, 4H, ph'-2,6H, ph'”-2,6H), $7.42(\mathrm{~d}, J=8.2 \mathrm{~Hz}, 2 \mathrm{H}, \mathrm{ph}-3,5 H), 7.25$ (t, $J=7.7 \mathrm{~Hz}, 2 \mathrm{H}, \mathrm{ph}$ '-3,5H), 7.12 (t, $J=7.3 \mathrm{~Hz}, 1 \mathrm{H}, \mathrm{ph}$ '-4H), 7.06 (t, $J=8.9 \mathrm{~Hz}, 2 \mathrm{H}, \mathrm{ph}$ ' $-3,5 H)$, 
$5.33(\mathrm{~s}, 1 \mathrm{H}, \mathrm{COH}), 3.83\left(\mathrm{~s}, 3 \mathrm{H}, \mathrm{OCH}_{3}\right), 3.49\left(\mathrm{~s}, 2 \mathrm{H}, \mathrm{NCH}_{2}\right), 2.77\left(\mathrm{~d}, J=8.1 \mathrm{~Hz}, 2 \mathrm{H}\right.$, pip-2, $\left.6 H^{e q}\right)$, $2.48(\mathrm{t}, J=11.8 \mathrm{~Hz}, 1 \mathrm{H}$, pip- $4 H), 1.95\left(\mathrm{t}, J=11.8 \mathrm{~Hz}, 2 \mathrm{H}\right.$, pip-2,6 $\left.H^{a x}\right), 1.52-1.44$ (m, 2H, pip$\left.3,5 H^{a x}\right), 1.21\left(\mathrm{t}, J=11.5 \mathrm{~Hz}, 2 \mathrm{H}\right.$, pip-3,5H $\left.H^{e q}\right) .{ }^{13} \mathrm{C}$ NMR (DMSO- $\left.d_{6}, 150 \mathrm{MHz}\right): \delta 166.2(C=\mathrm{O})$, 160. $5\left(\mathrm{~d},{ }^{1} J_{\mathrm{C}, \mathrm{F}}=240.6 \mathrm{~Hz}, \mathrm{ph}\right.$ '- $\left.-4 C\right), 147.1$ (ph'-1C), 144.7 (ph-4C), $143.5\left(\mathrm{~d},{ }^{4} J_{\mathrm{C}, \mathrm{F}}=2.7 \mathrm{~Hz}\right.$, ph'-1C), 129.1 (ph-2,6C), 128.8 (ph'-3,5C), 128.2 (ph-1C), 127.9 (ph-3,5C), 127.7 (d, ${ }^{3} J_{\mathrm{C}, \mathrm{F}}=$ $7.7 \mathrm{~Hz}, \mathrm{ph}$ '-2,6C), 125.9 (ph'-4C), 125.7 (ph'-2,6C), 114.4 (d, ${ }^{2} J_{\mathrm{C}, \mathrm{F}}=20.7 \mathrm{~Hz}, \mathrm{ph}$ '-3,5C), 78.3 $(\mathrm{COH}), 61.9\left(\mathrm{NCH}_{2}\right), 53.5$ (pip-2,6C), $52.1\left(\mathrm{OCH}_{3}\right), 43.3$ (pip-4C), 26.0 (pip-3,5C). ${ }^{19} \mathrm{~F}$ NMR (DMSO- $\left.d_{6}, 282 \mathrm{MHz}\right): \delta-118.08(\mathrm{ph}-F)$. HRMS $(\mathrm{m} / \mathrm{z}):[\mathrm{M}+\mathrm{H}]^{+}$calcd. for $\mathrm{C}_{27} \mathrm{H}_{28} \mathrm{FNO}_{3}$, 434.2131; found, 434.2122.

\section{4-((4-((4-Fluorophenyl)(hydroxy)(phenyl)methyl)piperidin-1-yl)methyl)benzoic acid (15).}

Compound $14(0.18 \mathrm{~g}, 0.4 \mathrm{mmol})$ was dissolved in methanol $(0.5 \mathrm{ml})$ and THF $(0.5 \mathrm{ml})$. Aqueous potassium hydroxide $(2 \mathrm{M}, 1 \mathrm{ml})$ was added and the mixture was stirred overnight at room temperature. The organic solvents were evaporated and the aqueous solution neutralized with hydrogen chloride solution. The product precipitated as a white solid that was recrystallized from acetonitrile under addition of a drop of water $(0.06 \mathrm{~g}, 35 \%)$. Mp: $>250{ }^{\circ} \mathrm{C} .{ }^{1} \mathrm{H}$ NMR (DMSO- $\left.d_{6}, 600 \mathrm{MHz}\right): \delta 7.86(\mathrm{~d}, J=8.1 \mathrm{~Hz}, 2 \mathrm{H}, \mathrm{ph}-2,6 H), 7.53-7.48$ (m, 4H, ph'-2,6H, ph'”2,6H), $7.39(\mathrm{~d}, J=8.1 \mathrm{~Hz}, 2 \mathrm{H}, \mathrm{ph}-3,5 H), 7.25(\mathrm{t}, J=7.7 \mathrm{~Hz}, 2 \mathrm{H}, \mathrm{ph}-3,5 H), 7.12(\mathrm{t}, J=7.3 \mathrm{~Hz}$, 1H, ph'-4H), 7.06 (t, $J=8.9 \mathrm{~Hz}, 2 \mathrm{H}, \mathrm{ph}$ '-3,5H), $5.34(\mathrm{~s}, 1 \mathrm{H}, \mathrm{OH}), 3.49$ (s, $\left.2 \mathrm{H}, \mathrm{NCH} H_{2}\right), 2.78$ (d, $J$ $=8.0 \mathrm{~Hz}, 2 \mathrm{H}$, pip-2,6 $\left.6 H^{e q}\right), 2.48(\mathrm{t}, J=11.9 \mathrm{~Hz}, 1 \mathrm{H}$, pip- $4 H), 1.96(\mathrm{t}, J=11.7 \mathrm{~Hz}, 2 \mathrm{H}$, pip2,6 $\left.6 H^{a x}\right), 1.51-1.45\left(\mathrm{~m}, 2 \mathrm{H}, \mathrm{pip}-3,5 H^{a x}\right), 1.21\left(\mathrm{~d}, J=11.1 \mathrm{~Hz}, 2 \mathrm{H}, \mathrm{pip}-3,5 H^{e q}\right) .{ }^{13} \mathrm{C}$ NMR (DMSO$\left.d_{6}, 150 \mathrm{MHz}\right): \delta 167.3(C=\mathrm{O}), 160.5\left(\mathrm{~d},{ }^{1} J_{\mathrm{C}, \mathrm{F}}=240.8 \mathrm{~Hz}, \mathrm{ph} "-4 C\right), 143.9(\mathrm{ph}-4 C), 143.5\left(\mathrm{~d},{ }^{4} J_{\mathrm{C}, \mathrm{F}}\right.$ $=2.6 \mathrm{~Hz}, \mathrm{ph}$ '-1C), 129.5 (ph-1C), $129.2(\mathrm{ph}-2,6 C), 128.7$ (ph'-3,5C), 127.9 (ph-3,5C), 127.7 (d, ${ }^{3} J_{\mathrm{C}, \mathrm{F}}=7.8 \mathrm{~Hz}, \mathrm{ph}$ '-2,6C), 125.9 (ph'-4C), 125.7 (ph'-2,6C), 114.4 (d, ${ }^{2} J_{\mathrm{C}, \mathrm{F}}=20.7 \mathrm{~Hz}, \mathrm{ph}$ '- 
3,5C), $78.3(\mathrm{COH}), 61.9\left(\mathrm{NCH}_{2}\right), 53.5$ (pip-2,6C), 43.3 (pip-4C), 25.9 (pip-3,5C). ${ }^{19} \mathrm{~F}$ NMR $\left(\mathrm{DMSO}-d_{6}, 282 \mathrm{MHz}\right): \delta-118.09(\mathrm{ph}-F)$. HRMS $(\mathrm{m} / \mathrm{z}):[\mathrm{M}+\mathrm{H}]^{+}$calcd. for $\mathrm{C}_{26} \mathrm{H}_{26} \mathrm{FNO}_{3}$, 420.1975; found, 420.1964. Elemental analysis: calcd. for $\mathrm{C}_{26} \mathrm{H}_{26} \mathrm{FNO}_{3} \cdot 0.25 \mathrm{H}_{2} \mathrm{O}, \mathrm{C} 73.65, \mathrm{H}$ 6.30, N 3.30; found, C 73.39, H 6.13, N 3.27.

Methyl 4-((4-(bis(4-fluorophenyl)(hydroxy)methyl)piperidin-1-yl)methyl)benzoate (16). Compound 35 (0.22 g, $0.65 \mathrm{mmol})$, methyl 4-(bromomethyl)benzoate (0.3 g, $1.3 \mathrm{mmol})$ and potassium carbonate $(0.18 \mathrm{~g}, 1.3 \mathrm{mmol})$ were suspended in acetone $(15 \mathrm{ml})$, refluxed overnight and stirred at room temperature for $24 \mathrm{~h}$. The inorganic components were filtered off and the filtrate concentrated under reduced pressure. Purification by column chromatography (TEA 0.5\%; DCM: methanol = 98: 2) gave the product as a colorless oil $(0.12 \mathrm{~g}, 41 \%) .{ }^{1} \mathrm{H}$ NMR (DMSO- $\left.d_{6}, 500 \mathrm{MHz}\right): \delta 7.87(\mathrm{~d}, J=8.3 \mathrm{~Hz}, 2 \mathrm{H}, \mathrm{ph}-2,6 H), 7.50-7.47(\mathrm{~m}, 4 \mathrm{H}, \mathrm{ph}-2,6 H), 7.40$ $(\mathrm{d}, J=8.2 \mathrm{~Hz}, 2 \mathrm{H}, \mathrm{ph}-3,5 H), 7.10\left(\mathrm{t}, J=8.9 \mathrm{~Hz}, 4 \mathrm{H}, \mathrm{ph}{ }^{\prime}-3,5 H\right), 5.38(\mathrm{~s}, 1 \mathrm{H}, \mathrm{COH}), 3.82(\mathrm{~s}, 3 \mathrm{H}$, $\left.\mathrm{OCH}_{3}\right), 3.47$ (s, 2H, $\left.\mathrm{NCH}_{2}\right), 2.75\left(\mathrm{~d}, J=10.9 \mathrm{~Hz}, 2 \mathrm{H}\right.$, pip-2,6H $\left.H^{e q}\right), 2.49-2.42$ (m, 1H, pip-4H), $1.94\left(\mathrm{t}, J=11.2 \mathrm{~Hz}, 2 \mathrm{H}, \mathrm{pip}-2,6 H^{a x}\right), 1.50-1.42\left(\mathrm{~m}, 2 \mathrm{H}, \mathrm{pip}-3,5 H^{a x}\right), 1.21(\mathrm{~d}, J=12.6 \mathrm{~Hz}, 2 \mathrm{H}$, pip-3,5H $\left.H^{e q}\right) .{ }^{13} \mathrm{C}$ NMR (DMSO- $\left.d_{6}, 125 \mathrm{MHz}\right): \delta 166.2(C=\mathrm{O}), 160.5\left(\mathrm{~d},{ }^{1} J_{\mathrm{C}, \mathrm{F}}=241.3 \mathrm{~Hz}\right.$, ph'4C), 144.6 (ph-4C), 143.3 (ph'-1C), 129.1 (ph-2,6C), $128.8(\mathrm{ph}-3,5 C), 127.7\left(\mathrm{~d},{ }^{3} J_{\mathrm{C}, \mathrm{F}}=7.8 \mathrm{~Hz}\right.$, ph'-2,6C), $114.4\left(\mathrm{~d},{ }^{2} J_{\mathrm{C}, \mathrm{F}}=20.9 \mathrm{~Hz}, \mathrm{ph}\right.$ '-3,5C), $78.1(\mathrm{COH}), 61.8\left(\mathrm{NCH}_{2}\right), 53.4$ (pip-2,6C), 52.0 $\left(\mathrm{OCH}_{3}\right), 43.3$ (pip-4C), 25.9 (pip-3,5C). ${ }^{19} \mathrm{~F}$ NMR (DMSO- $\left.d_{6}, 282 \mathrm{MHz}\right): \delta-117.89$ (ph-F). $\operatorname{HRMS}(\mathrm{m} / \mathrm{z})$ : $[\mathrm{M}]^{+}$calcd. for $\mathrm{C}_{27} \mathrm{H}_{27} \mathrm{~F}_{2} \mathrm{NO}_{3}, 452.2037$; found, 452.2024.

\section{4-((4-(Bis(4-fluorophenyl)(hydroxy)methyl)piperidin-1-yl)methyl)benzoic acid (17).}

Compound 16 (0.5 g, $1.1 \mathrm{mmol})$ was dissolved in methanol $(1 \mathrm{ml})$ and THF $(1 \mathrm{ml})$. Aqueous potassium hydroxide $(2 \mathrm{M}, 2 \mathrm{ml})$ was added and the mixture was heated in the microwave oven for $15 \mathrm{~min}$ at $70^{\circ} \mathrm{C}$. After cooling the organic solvents were evaporated and the aqueous solution 
neutralized with $\mathrm{HCl}$. The product precipitated as a white solid that was recrystallized from acetonitrile under addition of a drop of water $(0.15 \mathrm{~g}, 31 \%)$. Mp: $>250{ }^{\circ} \mathrm{C} .{ }^{1} \mathrm{H}$ NMR (DMSO- $d_{6}$, $500 \mathrm{MHz}): \delta 7.85(\mathrm{~d}, J=8.2 \mathrm{~Hz}, 2 \mathrm{H}, \mathrm{ph}-2,6 H), 7.50-7.48\left(\mathrm{~m}, 4 \mathrm{H}, \mathrm{ph}{ }^{\prime}-2,6 H\right), 7.38(\mathrm{~d}, J=8.2 \mathrm{~Hz}$, 2H, ph-3,5H), 7.09-7.04 (m, 4H, ph'-3,5H), $5.38(\mathrm{~s}, 1 \mathrm{H}, \mathrm{OH}), 3.48\left(\mathrm{~s}, 2 \mathrm{H}, \mathrm{NCH}_{2}\right), 2.76(\mathrm{~d}, J=$ $11.1 \mathrm{~Hz}, 2 \mathrm{H}$, pip-2,6 $\left.6 H^{e q}\right), 2.45(\mathrm{t}, J=11.8 \mathrm{~Hz}, 1 \mathrm{H}, \operatorname{pip}-4 H), 1.96\left(\mathrm{t}, J=11.0 \mathrm{~Hz}, 2 \mathrm{H}, \mathrm{pip}-2,6 H^{a x}\right)$, 1.50-1.42 (m, 2H, pip-3,5H $\left.H^{a x}\right), 1.21\left(\mathrm{~d}, J=12.5 \mathrm{~Hz}, 2 \mathrm{H}, \mathrm{pip}-3,5 H^{e q}\right) .{ }^{13} \mathrm{C}$ NMR (DMSO-d 6,125 MHz): $\delta 167.3(C=\mathrm{O}), 160.5\left(\mathrm{~d},{ }^{1} J_{\mathrm{C}, \mathrm{F}}=241.0 \mathrm{~Hz}, \mathrm{ph}\right.$ '-4C), $143.8(\mathrm{ph}-4 C), 143.2(\mathrm{ph}-1 C), 129.5$ (ph-1C), $129.2(\mathrm{ph}-2,6 C), 128.7(\mathrm{ph}-3,5 C), 127.7\left(\mathrm{~d},{ }^{3} J_{\mathrm{C}, \mathrm{F}}=7.9 \mathrm{~Hz}, \mathrm{ph}-2,6 C\right), 114.4\left(\mathrm{~d},{ }^{2} J_{\mathrm{C}, \mathrm{F}}=\right.$ $20.8 \mathrm{~Hz}$, ph'-3,5C), $78.1(\mathrm{COH}), 61.8\left(\mathrm{NCH}_{2}\right), 53.4$ (pip-2,6C), 43.3 (pip-4C), 25.9 (pip-3,5C). ${ }^{19} \mathrm{~F}$ NMR (DMSO- $\left.d_{6}, 282 \mathrm{MHz}\right): \delta-117.89(\mathrm{ph}-F)$. HRMS $(\mathrm{m} / \mathrm{z}):[\mathrm{M}+\mathrm{H}]^{+}$calcd. for $\mathrm{C}_{26} \mathrm{H}_{25} \mathrm{~F}_{2} \mathrm{NO}_{3}$, 438.1881; found, 438.1884. Elemental analysis: calcd. for $\mathrm{C}_{26} \mathrm{H}_{25} \mathrm{~F}_{2} \mathrm{NO}_{3} \cdot 0.25$ $\mathrm{H}_{2} \mathrm{O}, \mathrm{C} 70.65, \mathrm{H} 5.82, \mathrm{~N} 3.17$; found, C 70.67, H 5.67, N 3.25.

3-Fluorodihydrofuran-2(3H)-one (18). Under inert atmosphere, 3-hydroxy-dihydro-furan-2one (2.0 g, $19.6 \mathrm{mmol})$ was dissolved in DCM (30 ml). DAST (3.9 ml, $29.4 \mathrm{mmol})$ was added dropwise at $0{ }^{\circ} \mathrm{C}$. The solution was allowed to come to room temperature and stirred for $3 \mathrm{~h}$ at this temperature. The mixture was diluted with DCM and quenched with sodium bicarbonate solution at $0{ }^{\circ} \mathrm{C}$. The separated organic layer was washed with brine and water, dried $\left(\mathrm{MgSO}_{4}\right)$, filtered and concentrated to dryness. The product was purified by column chromatography (DCM) and was collected as colorless oil (1.15 g, 58\%). ${ }^{1} \mathrm{H} \mathrm{NMR}\left(\mathrm{CDCl}_{3}, 500 \mathrm{MHz}\right): \delta$ $5.23 / 5.13(\mathrm{t} / \mathrm{t}, J=7.7 / 7.7 \mathrm{~Hz}, 0.5 \mathrm{H} / 0.5 \mathrm{H}, \mathrm{CHF}), 4.50-4.46 / 4.32-4.27\left(\mathrm{~m} / \mathrm{m}, 1 \mathrm{H} / 1 \mathrm{H}, \mathrm{CH} \mathrm{H}_{2} \mathrm{O}\right), 2.72-$ 2.65/2.54-2.45 (m/m, 1H/1H, $\left.\mathrm{CHFCH}_{2}\right) .{ }^{13} \mathrm{C} \mathrm{NMR}\left(\mathrm{CDCl}_{3}, 125 \mathrm{MHz}\right): \delta 171.8\left(\mathrm{~d},{ }^{2} J_{\mathrm{C}, \mathrm{F}}=20.9\right.$ $\mathrm{Hz}, C=\mathrm{O}), 85.3\left(\mathrm{~d},{ }^{1} J_{\mathrm{C}, \mathrm{F}}=188.8 \mathrm{~Hz}, C \mathrm{HF}\right), 64.9\left(\mathrm{~d},{ }^{3} J_{\mathrm{C}, \mathrm{F}}=5.9 \mathrm{~Hz}, C \mathrm{H}_{2} \mathrm{O}\right), 29.5\left(\mathrm{~d},{ }^{2} J_{\mathrm{C}, \mathrm{F}}=20.0\right.$ 
$\left.\mathrm{Hz}, \mathrm{CHFCH} \mathrm{C}_{2}\right) .{ }^{19} \mathrm{~F} \mathrm{NMR}\left(\mathrm{CDCl}_{3}, 282 \mathrm{MHz}\right): \delta-196.16(\mathrm{CH} F) . \mathrm{HRMS}(\mathrm{m} / \mathrm{z}):[\mathrm{M}+\mathrm{H}]^{+}$calcd. for $\mathrm{C}_{4} \mathrm{H}_{5} \mathrm{FO}_{2}, 105.0352$; found, 105.0348 .

Methyl 2-fluoro-4-hydroxybutanoate (19). Compound 18 (1.73 g, 16.6 mmol) was dissolved in methanol $(75 \mathrm{ml})$. Sodium methanolate $(2.2 \mathrm{~g}, 41.5 \mathrm{mmol})$ was added in one portion. After stirring the solution for $1 \mathrm{~h}$ at room temperature it was quenched with $\mathrm{HCl}(2 \mathrm{~N})$. Methanol was evaporated under reduced pressure and the product was subsequently extracted into ethyl acetate. Purification was performed by column chromatography (DCM: ethyl acetate $=10: 0 \rightarrow 9: 1)$ giving the product as colorless oil $(0.58 \mathrm{~g}, 26 \%) .{ }^{1} \mathrm{H}$ NMR $\left(\mathrm{CDCl}_{3}, 300 \mathrm{MHz}\right): \delta 5.20 / 5.04$ $(\mathrm{dd} / \mathrm{dd}, J=7.3 / 7.0 \mathrm{~Hz}, 0.5 \mathrm{H} / 0.5 \mathrm{H}, \mathrm{CHF}), 3.90-3.70\left(\mathrm{~m}, 2 \mathrm{H}, \mathrm{CH}_{2} \mathrm{OH}\right), 3.80\left(\mathrm{~s}, 3 \mathrm{H}, \mathrm{OCH}_{3}\right), 2.26-$ $2.08\left(\mathrm{~m}, 2 \mathrm{H}, \mathrm{CHFCH}_{2}\right) .{ }^{13} \mathrm{C} \mathrm{NMR}\left(\mathrm{CDCl}_{3}, 150 \mathrm{MHz}\right): \delta 170.8\left(\mathrm{~d},{ }^{2} J_{\mathrm{C}, \mathrm{F}}=23.4 \mathrm{~Hz}, \mathrm{C}=\mathrm{O}\right), 86.5$ $\left(\mathrm{d},{ }^{1} J_{\mathrm{C}, \mathrm{F}}=168.5 \mathrm{~Hz}, C \mathrm{HF}\right), 57.8\left(\mathrm{~d},{ }^{3} J_{\mathrm{C}, \mathrm{F}}=4.1 \mathrm{~Hz}, C \mathrm{H}_{2} \mathrm{OH}\right), 52.8\left(\mathrm{OCH}_{3}\right), 35.1\left(\mathrm{~d},{ }^{2} J_{\mathrm{C}, \mathrm{F}}=20.6\right.$ $\left.\mathrm{Hz}, \mathrm{CHFCH} \mathrm{CH}_{2}\right) .{ }^{19} \mathrm{~F} \mathrm{NMR}\left(\mathrm{CDCl}_{3}, 282 \mathrm{MHz}\right): \delta-195.16(\mathrm{CH} F)$. HRMS (m/z): $[\mathrm{M}+\mathrm{H}]^{+}$calcd. for $\mathrm{C}_{5} \mathrm{H}_{9} \mathrm{FO}_{3}, 137.0614$; found, 137.0610 .

Methyl 2-fluoro-4-(tosyloxy)butanoate (20). Compound 19 (0.78 g, 5.9 mmol) was dissolved in DCM $(30 \mathrm{ml})$ and the solution was cooled to $0{ }^{\circ} \mathrm{C}$. Pyridine $(0.51 \mathrm{ml}, 6.2 \mathrm{mmol})$ and tosyl chloride (1.2 g, $6.2 \mathrm{mmol})$ were subsequently added. The mixture was stirred overnight at room temperature. It was diluted with DCM and the reaction was quenched with water. The separated organic layer was washed with brine, dried $\left(\mathrm{MgSO}_{4}\right)$ and concentrated under reduced pressure. The product was purified by column chromatography (DCM) giving a colorless oil (0.43 g, 25\%). ${ }^{1} \mathrm{H}$ NMR $\left(\mathrm{CDCl}_{3}, 600 \mathrm{MHz}\right): \delta 7.78(\mathrm{~d}, J=8.3 \mathrm{~Hz}, 2 \mathrm{H}, \mathrm{ph}-3,5 H), 7.37(\mathrm{~d}, J=8.0 \mathrm{~Hz}, 2 \mathrm{H}$, ph-2,6H), 5.03/4.95 (dd/dd, $J=8.4 / 8.5 \mathrm{~Hz}, 0.5 \mathrm{H} / 0.5 \mathrm{H}, \mathrm{CHF}), 4.19-4.15\left(\mathrm{~m}, 2 \mathrm{H}, \mathrm{CH}_{2} \mathrm{O}\right), 3.78(\mathrm{~s}$, $\left.3 \mathrm{H}, \mathrm{OCH}_{3}\right), 2.45\left(\mathrm{~s}, 3 \mathrm{H}, \mathrm{ph}-\mathrm{CH}_{3}\right), 2.36-2.15\left(\mathrm{~m}, 2 \mathrm{H}, \mathrm{CHFCH}_{2}\right) \cdot{ }^{13} \mathrm{C} \mathrm{NMR}\left(\mathrm{CDCl}_{3}, 150 \mathrm{MHz}\right): \delta$ $169.5\left(\mathrm{~d},{ }^{2} J_{\mathrm{C}, \mathrm{F}}=23.3 \mathrm{~Hz}, C=\mathrm{O}\right), 145.3(\mathrm{ph}-4 C), 132.6(\mathrm{ph}-1 C), 130.1(\mathrm{ph}-2,6 C), 128.1(\mathrm{ph}-$ 
3,5C), $85.0\left(\mathrm{~d},{ }^{1} J_{\mathrm{C}, \mathrm{F}}=184.4 \mathrm{~Hz}, \mathrm{CHF}\right), 64.9\left(\mathrm{~d},{ }^{3} J_{\mathrm{C}, \mathrm{F}}=3.6 \mathrm{~Hz}, \mathrm{CH}_{2} \mathrm{O}\right), 52.8\left(\mathrm{OCH}_{3}\right), 32.1(\mathrm{~d}$, $\left.{ }^{2} J_{\mathrm{C}, \mathrm{F}}=20.9 \mathrm{~Hz}, \mathrm{CHFCH} \mathrm{H}_{2}\right), 21.8\left(\mathrm{ph}-\mathrm{CH}_{3}\right) .{ }^{19} \mathrm{~F} \mathrm{NMR}\left(\mathrm{CDCl}_{3}, 282 \mathrm{MHz}\right): \delta-195.79(\mathrm{CH} F)$. HRMS (m/z): $[\mathrm{M}+\mathrm{H}]^{+}$calcd. for $\mathrm{C}_{12} \mathrm{H}_{15} \mathrm{FO}_{5} \mathrm{~S}, 291.0703$; found, 291.0692 .

Methyl 4-(4-benzhydrylpiperazin-1-yl)-2-fluorobutanoate (21). TEA $(0.2 \mathrm{ml}, 0.5 \mathrm{mmol})$ was dissolved in dry acetonitrile. Under inert atmosphere, 1-benzhydryl-piperazine ( $0.37 \mathrm{~g}, 1.49$ mmol) and compound 20 (0.43 g, $1.49 \mathrm{mmol})$ were subsequently added. The solution was stirred for $6 \mathrm{~h}$ at $70{ }^{\circ} \mathrm{C}$. After cooling it was concentrated to dryness and the product was purified by column chromatography (TEA; DCM: methanol = 99: 1) giving a light yellow oil (0.22 g, 40\%). ${ }^{1} \mathrm{H}$ NMR (DMSO-d $\left.d_{6}, 600 \mathrm{MHz}\right): \delta 7.40(\mathrm{~d}, J=7.4 \mathrm{~Hz}, 4 \mathrm{H}, \mathrm{ph}-2,6 H), 7.28(\mathrm{t}, J=7.6 \mathrm{~Hz}, 4 \mathrm{H}$, ph3,5H), 7.17 (t, $J=7.4 \mathrm{~Hz}, 2 \mathrm{H}, \mathrm{ph}-4 H), 5.17 / 5.09$ (t/t, $J=5.4 / 5.4 \mathrm{~Hz}, 0.5 \mathrm{H} / 0.5 \mathrm{H}, \mathrm{CHF}), 4.25$ (s, 1H, benzh.-CH), $3.67\left(\mathrm{~s}, 3 \mathrm{H}, \mathrm{OCH}_{3}\right), 2.50-2.15\left(\mathrm{~m}, 10 \mathrm{H}\right.$, prz-2,3,5,6H, $\left.\mathrm{NCH}_{2}\right), 1.98-1.93(\mathrm{~m}$, $\left.2 \mathrm{H}, \mathrm{CHFCH}_{2}\right) .{ }^{13} \mathrm{C}$ NMR (DMSO- $\left.d_{6}, 150 \mathrm{MHz}\right): \delta 169.9\left(\mathrm{~d},{ }^{2} J_{\mathrm{C}, \mathrm{F}}=23.7 \mathrm{~Hz}, \mathrm{C}=\mathrm{O}\right), 142.9(\mathrm{ph}-$ 1C), 128.5 (ph-3,5C), 127.6 (ph-2,6C), 126.9 (ph-4C), 87.0 (d, $\left.{ }^{1} J_{\mathrm{C}, \mathrm{F}}=179.7 \mathrm{~Hz}, C \mathrm{HF}\right), 75.1$ (benzh.- $\mathrm{CH}$ ), $55.0\left(\mathrm{NCH}_{2}\right), 52.8$ (prz-2,6C), $52.0\left(\mathrm{OCH}_{3}\right), 51.5$ (prz-3,5C), $29.1\left(\mathrm{~d},{ }^{2} J_{\mathrm{C}, \mathrm{F}}=20.6\right.$ $\mathrm{Hz}, \mathrm{CHFCH}_{2}$ ). ${ }^{19} \mathrm{~F}$ NMR (DMSO- $\left.d_{6}, 282 \mathrm{MHz}\right): \delta-193.32(\mathrm{CH} F)$. HRMS (m/z): $[\mathrm{M}+\mathrm{H}]^{+}$calcd. for $\mathrm{C}_{22} \mathrm{H}_{27} \mathrm{FN}_{2} \mathrm{O}_{2}, 371.2135$; found, 371.2140 .

4-(4-Benzhydrylpiperazin-1-yl)-2-fluorobutan-1-ol dihydrochloride (22). Lithium aluminium hydride $(0.031 \mathrm{~g}, 0.81 \mathrm{mmol})$ was dissolved in THF $(5 \mathrm{ml})$. Compound $21(0.1 \mathrm{~g}$, $0.27 \mathrm{mmol}$ ), dissolved in THF ( $5 \mathrm{ml}$ ), was added dropwise under inert atmosphere. The mixture was stirred $1 \mathrm{~h}$ at room temperature and subsequently quenched with brine. THF was evaporated and the aqueous solution was adjusted to $\mathrm{pH}=10$ using aqueous $\mathrm{NaOH}(2 \mathrm{~N})$. The product was extracted into DCM. Drying $\left(\mathrm{MgSO}_{4}\right)$ and concentration afforded a colorless oil (0.08 g, 87\%). The product was crystallised with $\mathrm{HCl}$ in ethanol and precipitated as white solid $(0.046 \mathrm{~g}, 42 \%)$. 
Mp: $209{ }^{\circ} \mathrm{C} .{ }^{1} \mathrm{H}$ NMR (DMSO-d $\left.d_{6}, 400 \mathrm{MHz}, 80{ }^{\circ} \mathrm{C}\right): \delta 7.68(\mathrm{~d}, J=7.2 \mathrm{~Hz}, 4 \mathrm{H}, \mathrm{ph}-2,6 H), 7.38(\mathrm{t}$, $J=7.5 \mathrm{~Hz}, 4 \mathrm{H}, \mathrm{ph}-3,5 H), 7.29$ (t, $J=7.3 \mathrm{~Hz}, 2 \mathrm{H}, \mathrm{ph}-4 H$ ), 5.09 (br s, 1H, benzh.-CH), 4.714.66/4.59-4.54 (m/m, 0.5H/0.5H, CHF), 3.64-3.53 (m, 2H, $\left.\mathrm{CH}_{2} \mathrm{OH}\right), 3.48(\mathrm{~s}, 4 \mathrm{H}$, prz-2,6H $)$, $3.23\left(\mathrm{t}, J=8.0 \mathrm{~Hz}, 2 \mathrm{H}, \mathrm{NCH} \mathrm{H}_{2}\right), 3.05\left(\mathrm{~s}, 4 \mathrm{H}, \mathrm{prz}-3,5 \mathrm{H}_{2}\right), 2.18-2.07\left(\mathrm{~m}, 2 \mathrm{H}, \mathrm{CHFCH}_{2}\right) .{ }^{13} \mathrm{C} \mathrm{NMR}$ (DMSO- $\left.d_{6}, 150 \mathrm{MHz}\right): \delta 135.3(\mathrm{ph}-1 C), 129.3(\mathrm{ph}-3-5 C), 128.2(\mathrm{ph}-2,6 C), 92.1\left(\mathrm{~d},{ }^{1} J_{\mathrm{C}, \mathrm{F}}=169.1\right.$ $\mathrm{Hz}, C \mathrm{HF}$ ), 74.1 (benzh.- $C \mathrm{H}), 62.5\left(\mathrm{~d},{ }^{2} J_{\mathrm{C}, \mathrm{F}}=19.7 \mathrm{~Hz}, \mathrm{CH}_{2} \mathrm{OH}\right), 52.1\left(\mathrm{NCH}_{2}\right), 48.5$ (prz-2,6C), 44.2 (prz-3,5C), $25.2\left(\mathrm{CHFCH}_{2}\right) .{ }^{19} \mathrm{~F} \mathrm{NMR}\left(\mathrm{CDCl}_{3}, 282 \mathrm{MHz}\right): \delta-188.20(\mathrm{CH} F) . \mathrm{HRMS}(\mathrm{m} / \mathrm{z})$ : $[\mathrm{M}+\mathrm{H}]^{+}$calcd. for $\mathrm{C}_{21} \mathrm{H}_{27} \mathrm{FN}_{2} \mathrm{O}$, 343.2186; found, 343.2184. Elemental analysis: calcd. for $\mathrm{C}_{21} \mathrm{H}_{27} \mathrm{FN}_{2} \mathrm{O} \cdot 2 \mathrm{HCl} \cdot 0.25 \mathrm{H}_{2} \mathrm{O}, \mathrm{C} 60.07, \mathrm{H} 7.08, \mathrm{~N}$ 6.69; found, $\mathrm{C} 60.72, \mathrm{H} 7.04, \mathrm{~N}$ 6.74.

4-(4-Benzhydrylpiperazin-1-yl)-2-fluorobutyl acetate (23). Compound 22 (liberated base; $0.1 \mathrm{~g}, 0.29 \mathrm{mmol})$ was dissolved in DCM $(5 \mathrm{ml})$. TEA $(43 \mu \mathrm{l}, 0.3 \mathrm{mmol})$ and acetic anhydride (35 $\mu \mathrm{l}, 0.3 \mathrm{mmol})$ were added under cooling (ice bath). The mixture was stirred $3 \mathrm{~h}$ at room temperature and subsequently quenched with aqueous $\mathrm{NaOH}(2 \mathrm{M})$. The organic layer was dried $\left(\mathrm{MgSO}_{4}\right)$, filtered and purified by column chromatography $(\mathrm{TEA}$; DCM: methanol = 99.5: 0.5). The product was isolated as colorless oil (0.09 g, 80\%). ${ }^{1} \mathrm{H}$ NMR (DMSO- $\left.d_{6}, 300 \mathrm{MHz}\right): \delta 7.41$ (d, $J=7.3 \mathrm{~Hz}, 4 \mathrm{H}, \mathrm{ph}-2,6 H), 7.27$ (t, $J=7.5 \mathrm{~Hz}, 4 \mathrm{H}, \mathrm{ph}-3,5 H), 7.16(\mathrm{t}, J=7.2 \mathrm{~Hz}, 2 \mathrm{H}, \mathrm{ph}-4 H)$, 4.83-4.63 (m, 1H, CHF), 4.23 (s, 1H, benzh.-CH), 4.17-4.03 (m, 2H, $\left.\mathrm{CH}_{2} \mathrm{OCOCH}_{3}\right), 2.52-2.26$ (m, 10H, prz-2,3,5,6H, $\left.\mathrm{NCH}_{2}\right), 2.02\left(\mathrm{~s}, 3 \mathrm{H}, \mathrm{COCH}_{3}\right), 1.81-1.65\left(\mathrm{~m}, 2 \mathrm{H}, \mathrm{CH}_{2} \mathrm{CH}\right) .{ }^{13} \mathrm{C}$ NMR $\left(\mathrm{DMSO}-d_{6}, 150 \mathrm{MHz}\right): \delta 170.2(C=\mathrm{O}), 143.0(\mathrm{ph}-1 C), 128.6(\mathrm{ph}-3,5 C), 127.6(\mathrm{ph}-2,6 C), 126.9$ (ph-4C), $90.1\left(\mathrm{~d},{ }^{1} J_{\mathrm{C}, \mathrm{F}}=170.0 \mathrm{~Hz}\right.$, but-2C), 75.2 (benzh.-CH), $65.4\left(\mathrm{~d},{ }^{2} J_{\mathrm{C}, \mathrm{F}}=20.4 \mathrm{~Hz}\right.$, but-1C), $53.0\left(\mathrm{~d},{ }^{3} J_{\mathrm{C}, \mathrm{F}}=5.2 \mathrm{~Hz}\right.$, but-4C), 52.8/52.7 (prz-2,6C), $51.6(\operatorname{prz}-3,5 C), 28.0\left(\mathrm{~d},{ }^{2} J_{\mathrm{C}, \mathrm{F}}=20.4 \mathrm{~Hz}\right.$, but-3C), $20.6\left(\mathrm{COCH}_{3}\right) .{ }^{19} \mathrm{~F}$ NMR (DMSO- $\left.d_{6}, 282 \mathrm{MHz}\right): \delta-186.59(\mathrm{CH} F) . \mathrm{HRMS}(\mathrm{m} / \mathrm{z})$ : $[\mathrm{M}+\mathrm{H}]^{+}$calcd. for $\mathrm{C}_{23} \mathrm{H}_{29} \mathrm{FN}_{2} \mathrm{O}_{2}, 384.2213$; found, 384.2210. 
4-(4-Benzhydrylpiperazin-1-yl)-2-fluorobutanoic acid (24). Compound 21 (0.1 g, 0.27 mmol) was dissolved in methanol $(0.5 \mathrm{ml})$ and THF $(0.5 \mathrm{ml})$. Aqueous potassium hydroxide $(2$ M, $1 \mathrm{ml}$ ) was added and the mixture was stirred overnight at room temperature. The organic solvents were evaporated and the aqueous solution neutralized with hydrogen chloride solution. The product precipitated as a white solid that was recrystallized from acetonitrile under addition of a drop of water $(0.05 \mathrm{~g}, 49 \%)$. Mp: $227-230{ }^{\circ} \mathrm{C}$ (carbonization). ${ }^{1} \mathrm{H}$ NMR (DMSO- $d_{6}, 600$ MHz): $\delta 7.38(\mathrm{~d}, J=7.6 \mathrm{~Hz}, 4 \mathrm{H}, \mathrm{ph}-2,6 H), 7.25(\mathrm{t}, J=7.5 \mathrm{~Hz}, 4 \mathrm{H}, \mathrm{ph}-3,5 H), 7.15(\mathrm{t}, J=7.2 \mathrm{~Hz}$, 2H, ph-4H), 4.95/4.87 (t/t, $J=5.8 / 5.8 \mathrm{~Hz}, 0.5 \mathrm{H} / 0.5 \mathrm{H}, \mathrm{CHF}), 4.39$ (s, 1H, benzh.-CH), 3.50 (br s, 4H, prz-2,6H $), 3.36-3.31 / 3.23-3.17\left(\mathrm{~m} / \mathrm{m}, 1 \mathrm{H} / 1 \mathrm{H}, \mathrm{NCH}_{2}\right), 2.99$ (br s, 4H, prz-3,5H2), 2.39-2.22 $\left(\mathrm{m}, 2 \mathrm{H}, \mathrm{CHFCH}{ }_{2}\right) .{ }^{13} \mathrm{C} \mathrm{NMR}\left(\mathrm{DMSO}-d_{6}, 150 \mathrm{MHz}\right): \delta 171.0\left(\mathrm{~d},{ }^{2} J_{\mathrm{C}, \mathrm{F}}=22.1 \mathrm{~Hz}, \mathrm{C}=\mathrm{O}\right), 141.6$ (ph-1C), 128.6 (ph-3,5C), $127.4(\mathrm{ph}-2,6 C), 127.1(\mathrm{ph}-4 C), 86.9\left(\mathrm{~d},{ }^{1} J_{\mathrm{C}, \mathrm{F}}=181.5 \mathrm{~Hz}, C \mathrm{HF}\right), 73.9$ (benzh.- $\mathrm{CH}$ ), $51.8\left(\mathrm{NCH}_{2}\right), 51.2$ (prz-2,6C), 48.2 (prz-3,5C), $26.6\left(\mathrm{~d},{ }^{2} J_{\mathrm{C}, \mathrm{F}}=22.1 \mathrm{~Hz}, \mathrm{CHFCH}_{2}\right)$. ${ }^{19} \mathrm{~F}$ NMR $\left(\mathrm{DMSO}-d_{6}, 282 \mathrm{MHz}\right): \delta-186.28(\mathrm{CH} F) . \operatorname{HRMS}(\mathrm{m} / \mathrm{z}):[\mathrm{M}+\mathrm{H}]^{+}$calcd. for $\mathrm{C}_{21} \mathrm{H}_{25} \mathrm{FN}_{2} \mathrm{O}_{2}$, 357.1978; found, 357.1980. HPLC (analytical Chromolith column (RP-18e, 100$4.6 \mathrm{~mm}), 3 \mathrm{ml} / \mathrm{min}$, methanol (0.1\% trifluoroacetic acid) $20-60 \%$ in $8 \mathrm{~min}$ ) RT: $5.2 \mathrm{~min}$; purity: $99.62 \%$.

\section{Methyl 2-fluoro-4-(4-(hydroxydiphenylmethyl)piperidin-1-yl)butanoate}

(25).

Diphenyl(piperidin-4-yl)methanol hydrochloride $(1.1 \mathrm{~g}, 3.6 \mathrm{mmol})$ was dissolved in DMSO (25 ml). TEA (1 ml, $7.2 \mathrm{mmol})$ and compound 20 (1.4 g, $4.8 \mathrm{mmol})$ were subsequently added. The mixture was stirred overnight at $60^{\circ} \mathrm{C}$. After cooling the product was extracted into DCM and purified by column chromatography (TEA; DCM: methanol = 99: 1) giving a light yellow oil $(0.35 \mathrm{~g}, 25 \%) .{ }^{1} \mathrm{H}$ NMR (DMSO- $\left.d_{6}, 600 \mathrm{MHz}\right): \delta 7.49(\mathrm{~d}, J=8.2 \mathrm{~Hz}, 4 \mathrm{H}, \mathrm{ph}-2,6 H), 7.25(\mathrm{t}, J=$ $7.7 \mathrm{~Hz}, 4 \mathrm{H}, \mathrm{ph}-3,5 H), 7.12(\mathrm{t}, J=7.3 \mathrm{~Hz}, 2 \mathrm{H}, \mathrm{ph}-4 H), 5.27(\mathrm{~s}, 1 \mathrm{H}, \mathrm{OH}), 5.16 / 5.08(\mathrm{t} / \mathrm{t}, J=$ 
5.4/5.4 Hz, 0.5H/0.5H, CHF), $3.67\left(\mathrm{~s}, 3 \mathrm{H}, \mathrm{CH}_{3}\right), 2.82-2.76\left(\mathrm{~m}, 2 \mathrm{H}, \mathrm{pip}-2,6 H^{\mathrm{eq}}\right), 2.45(\mathrm{t}, J=11.9$ $\mathrm{Hz}, 1 \mathrm{H}$, pip-4H), 2.36-2.30 (m, 2H, $\left.\mathrm{NCH}_{2}\right), 1.98-1.85$ (m, 4H, $\mathrm{CH}_{2} \mathrm{CHF}$, pip-2,6H ${ }^{\mathrm{ax}}$ ), 1.44-1.39 $\left(\right.$ pip-3,5H $\left.H^{\mathrm{eq}}\right), 1.25\left(\mathrm{~d}, J=12.0 \mathrm{~Hz}, 2 \mathrm{H}, \mathrm{pip}-3,5 H^{\mathrm{ax}}\right) .{ }^{13} \mathrm{C}$ NMR $\left(\mathrm{DMSO}-d_{6}, 150 \mathrm{MHz}\right): \delta 170.0(\mathrm{~d}$, $\left.{ }^{2} J_{\mathrm{C}, \mathrm{F}}=23.6 \mathrm{~Hz}, C=\mathrm{O}\right), 147.3(\mathrm{ph}-1 C), 127.8(\mathrm{ph}-3-5 C), 125.8(\mathrm{ph}-2,6 C), 87.1\left(\mathrm{~d},{ }^{1} J_{\mathrm{C}, \mathrm{F}}=179.7\right.$ $\mathrm{Hz}, C \mathrm{HF}), 78.5(\mathrm{COH}), 55.0\left(\mathrm{NCH}_{2}\right), 53.5\left(\right.$ pip-2,6C), $52.1\left(\mathrm{CH}_{3}\right), 43.4$ (pip-4C), $29.4\left(\mathrm{~d},{ }^{2} J_{\mathrm{C}, \mathrm{F}}=\right.$ $20.6 \mathrm{~Hz}, \mathrm{CHFCH}$ ), 26.0 (pip-3,5C). ${ }^{19} \mathrm{~F}$ NMR (DMSO- $\left.d_{6}, 282 \mathrm{MHz}\right): \delta-193.29(\mathrm{CH} F)$. HRMS $(\mathrm{m} / \mathrm{z}):[\mathrm{M}+\mathrm{H}]^{+}$calcd. for $\mathrm{C}_{23} \mathrm{H}_{28} \mathrm{FNO}_{3}, 386.2131$; found, 386.2121.

\section{2-Fluoro-4-(4-(hydroxydiphenylmethyl)piperidin-1-yl)butan-1-ol (26). Compound 25 (0.2} $\mathrm{g}, 0.52 \mathrm{mmol})$ in THF $(5 \mathrm{ml})$ was added dropwise to a suspension of lithium aluminium hydride (75 mg, $1.95 \mathrm{mmol})$ in THF $(5 \mathrm{ml})$. After stirring for $1 \mathrm{~h}$ at room temperature the solution was quenched with brine and concentrated to dryness. The crude mixture was taken up in aqueous $\mathrm{NaOH}(2 \mathrm{~N})$ and the product was extracted into DCM. Concentration gave the product as a white solid, which was purified by column chromatography (TEA; DCM: methanol = 9: 1); $(0.15 \mathrm{~g}$, 83\%). Mp: $144{ }^{\circ} \mathrm{C} .{ }^{1} \mathrm{H}$ NMR (DMSO- $\left.d_{6}, 600 \mathrm{MHz}\right): \delta 7.50(\mathrm{~d}, J=8.2 \mathrm{~Hz}, 4 \mathrm{H}, \mathrm{ph}-2,6 H), 7.25(\mathrm{t}$, $J=7.5 \mathrm{~Hz}, 4 \mathrm{H}, \mathrm{ph}-3,5 H), 7.12(\mathrm{t}, J=7.3 \mathrm{~Hz}, 2 \mathrm{H}, \mathrm{ph}-4 H), 5.26(\mathrm{~s}, 1 \mathrm{H}, \mathrm{OH}), 5.00$ (br s, $1 \mathrm{H}$, $\left.\mathrm{CH}_{2} \mathrm{OH}\right), 4.56-4.52 / 4.48-4.44(\mathrm{~m} / \mathrm{m}, 0.5 \mathrm{H} / 0.5 \mathrm{H}, \mathrm{CHF}), 3.54-3.41\left(\mathrm{~m}, 2 \mathrm{H}, \mathrm{CH}_{2} \mathrm{OH}\right), 2.86$ (br s, 2H, pip-2,6H $H^{\mathrm{eq}}$ ), $2.46\left(\mathrm{t}, J=11.6 \mathrm{~Hz}, 1 \mathrm{H}\right.$, pip- $4 H$ ), 2.33 (br s, 2H, $\mathrm{NCH}_{2}$ ), 1.93-1.87 (m, 2H, pip2,6 $\left.H^{\mathrm{ax}}\right), 1.74-1.64\left(\mathrm{CH}_{2} \mathrm{CHF}\right), 1.50-1.42$ (pip-3,5H $\left.H^{\mathrm{eq}}\right), 1.25$ (br s, $2 \mathrm{H}$, pip-3,5H $\left.{ }^{\mathrm{ax}}\right) .{ }^{13} \mathrm{C}$ NMR (DMSO- $\left.d_{6}, 150 \mathrm{MHz}\right): \delta 147.3$ (ph-1C), 127.8 (ph-3-5C), $125.8(\mathrm{ph}-2,6 C), 93.3\left(\mathrm{~d},{ }^{1} J_{\mathrm{C}, \mathrm{F}}=167.6\right.$ $\mathrm{Hz}, C \mathrm{HF}), 78.5(\mathrm{COH}), 64.0\left(\mathrm{~d},{ }^{2} J_{\mathrm{C}, \mathrm{F}}=21.8 \mathrm{~Hz}, \mathrm{CH}_{2} \mathrm{OH}\right), 53.8(\mathrm{pip}-2,6 C), 53.3\left(\mathrm{NCH}_{2}\right), 43.4$ (pip-4C), $28.5\left(\mathrm{~d},{ }^{2} J_{\mathrm{C}, \mathrm{F}}=22.8 \mathrm{~Hz}, \mathrm{CH}_{2} \mathrm{CHF}\right.$ ), 25.9 (pip-3,5C). ${ }^{19} \mathrm{~F}$ NMR (DMSO-d $6,282 \mathrm{MHz}$ ): $\delta-186.46(\mathrm{CH} F)$. HRMS (m/z): $[\mathrm{M}+\mathrm{H}]^{+}$calcd. for $\mathrm{C}_{22} \mathrm{H}_{28} \mathrm{FNO}_{2}, 358.2182$; found, 358.2170. 


\section{2-Fluoro-4-(4-(hydroxydiphenylmethyl)piperidin-1-yl)butyl acetate (27). Compound 26}

(0.1 g, $0.28 \mathrm{mmol})$ was dissolved in DCM (5 ml). TEA (43 $\mu \mathrm{l}, 0.3 \mathrm{mmol})$ and acetic anhydride ( $35 \mu \mathrm{l}, 0.3 \mathrm{mmol}$ ) were added under cooling (ice bath). The mixture was stirred $3 \mathrm{~h}$ at room temperature and subsequently quenched with aqueous $\mathrm{NaOH}(2 \mathrm{~N})$. The organic layer was dried $\left(\mathrm{MgSO}_{4}\right)$, filtered and purified by column chromatography (TEA; DCM: methanol = 95: 5). The product was isolated as colorless oil $\left(0.11 \mathrm{~g}\right.$, quantitative conversion). ${ }^{1} \mathrm{H}$ NMR (DMSO- $d_{6}, 600$ MHz): $\delta 7.49(\mathrm{~d}, J=7.9 \mathrm{~Hz}, 4 \mathrm{H}, \mathrm{ph}-2,6 H), 7.25(\mathrm{t}, J=7.3 \mathrm{~Hz}, 4 \mathrm{H}, \mathrm{ph}-3,5 H), 7.11(\mathrm{t}, J=7.6 \mathrm{~Hz}$, $2 \mathrm{H}, \mathrm{ph}-4 H), 5.26(\mathrm{~s}, 1 \mathrm{H}, \mathrm{OH}), 4.80-4.77 / 4.72-4.68(\mathrm{~m} / \mathrm{m}, 0.5 \mathrm{H} / 0.5 \mathrm{H}, \mathrm{CHF}), 4.23-4.08(\mathrm{~m}, 2 \mathrm{H}$, $\left.\mathrm{CH}_{2} \mathrm{OAc}\right), 2.86-2.81\left(\mathrm{~m}, 2 \mathrm{H}, \mathrm{pip}-2,6 H^{\mathrm{eq}}\right), 2.46(\mathrm{t}, J=11.8 \mathrm{~Hz}, 1 \mathrm{H}, \mathrm{pip}-4 H), 2.37-2.30(\mathrm{~m}, 2 \mathrm{H}$, $\mathrm{NCH}_{2}$ ), 2.04 (s, 3H, $\left.\mathrm{COCH}_{3}\right), 1.91-1.69$ (m, 4H, $\mathrm{CH}_{2} \mathrm{CHF}$, pip-2,6H $\left.{ }^{\mathrm{ax}}\right), 1.49-1.44$ (pip-3,5H ${ }^{\mathrm{eq}}$ ), 1.21 (br s, $2 \mathrm{H}$, pip-3,5H ax $).{ }^{13} \mathrm{C}$ NMR (DMSO- $\left.d_{6}, 150 \mathrm{MHz}\right): \delta 170.2(C=\mathrm{O}), 147.3(\mathrm{ph}-1 C)$, $127.8(\mathrm{ph}-3-5 C), 125.8(\mathrm{ph}-2,6 C), 90.6\left(\mathrm{~d},{ }^{1} J_{\mathrm{C}, \mathrm{F}}=168.9 \mathrm{~Hz}, C \mathrm{HF}\right), 78.5(C \mathrm{OH}), 65.4\left(\mathrm{~d},{ }^{2} J_{\mathrm{C}, \mathrm{F}}=\right.$ $\left.20.4 \mathrm{~Hz}, \mathrm{CH}_{2} \mathrm{OAc}\right), 54.0\left(\mathrm{NCH}_{2}\right), 53.3$ (pip-2,6C), 43.5 (pip-4C), $28.2\left(\mathrm{~d},{ }^{2} J_{\mathrm{C}, \mathrm{F}}=20.3 \mathrm{~Hz}\right.$, $\mathrm{CH}_{2} \mathrm{CHF}$ ), 26.0 (pip-3,5C), $20.6\left(\mathrm{COCH}_{3}\right) .{ }^{19} \mathrm{~F}$ NMR (DMSO-d $\left.d_{6}, 282 \mathrm{MHz}\right): \delta-186.72(\mathrm{CH} F)$. HRMS (m/z): $[\mathrm{M}+\mathrm{H}]^{+}$calcd. for $\mathrm{C}_{24} \mathrm{H}_{30} \mathrm{FNO}_{3}, 400.2288$; found, 400.2286 .

\section{2-Fluoro-4-(4-(hydroxydiphenylmethyl)piperidin-1-yl)butanoic acid (28). Compound 25}

(0.13 $\mathrm{g}, 0.34 \mathrm{mmol})$ was dissolved in methanol $(0.5 \mathrm{ml})$ and THF $(0.5 \mathrm{ml})$. Aqueous potassium hydroxide ( $2 \mathrm{M}, 1 \mathrm{ml})$ was added and the mixture was stirred overnight at room temperature. The organic solvents were subsequently evaporated and the aqueous solution neutralized with hydrogen chloride solution. The product precipitated as a white solid that was recrystallized from acetonitrile under addition of a drop of water (0.03 g, 24\%). Mp: $242-244{ }^{\circ} \mathrm{C}$ (carbonisation). ${ }^{1} \mathrm{H}$ NMR (DMSO- $\left.d_{6}, 600 \mathrm{MHz}\right): \delta 7.43(\mathrm{~d}, J=7.7 \mathrm{~Hz}, 4 \mathrm{H}, \mathrm{ph}-2,6 H), 7.21(\mathrm{t}, J=7.7 \mathrm{~Hz}, 4 \mathrm{H}, \mathrm{ph}-$ 3,5H), $7.07(\mathrm{t}, J=7.3 \mathrm{~Hz}, 2 \mathrm{H}, \mathrm{ph}-4 H), 5.23(\mathrm{~s}, 1 \mathrm{H}, \mathrm{OH}), 4.92 / 4.84(\mathrm{~s} / \mathrm{s}, 0.5 \mathrm{H} / 0.5 \mathrm{H}, \mathrm{CHF}), 3.43$ 
(br s, 2H, pip-2,6H eq $), 3.13-3.04\left(\mathrm{~m}, 2 \mathrm{H}, \mathrm{NCH}_{2}\right), 2.91\left(\mathrm{~m}, 2 \mathrm{H}, \mathrm{pip}-2,6 H^{\mathrm{ax}}\right), 2.74(\mathrm{~s}, 1 \mathrm{H}$, pip-4H), 2.31-2.10 ( $\mathrm{CH}_{2} \mathrm{CHF}$ ), 1.49/1.25 (overlay with $\mathrm{CD}_{3} \mathrm{COOD}$ peak: pip-3,5 $\mathrm{H}_{2}$ ). ${ }^{13} \mathrm{C}$ NMR (DMSO$\left.d_{6}, 150 \mathrm{MHz}\right): \delta 171.0(C=\mathrm{O}), 146.0(\mathrm{ph}-1 C), 127.8$ (ph-3,5C), $126.0(\mathrm{ph}-4 C), 125.5$ (ph-2,6C), $86.8\left(\mathrm{~d},{ }^{1} J_{\mathrm{C}, \mathrm{F}}=181.8 \mathrm{~Hz}, C \mathrm{HF}\right), 77.9(\mathrm{COH}), 51.8\left(\right.$ pip-2,6C, $\left.\mathrm{NCH}_{2}\right), 40.5($ pip-4C), $26.6(\mathrm{~d}$, $\left.{ }^{2} J_{\mathrm{C}, \mathrm{F}}=21.6 \mathrm{~Hz}, C \mathrm{H}_{2} \mathrm{CHF}\right), 23.8$ (pip-3,5C). ${ }^{19} \mathrm{~F}$ NMR (DMSO-d $\left.6,282 \mathrm{MHz}\right): \delta-187.15(\mathrm{CH} F)$. HRMS (m/z): $[\mathrm{M}+\mathrm{H}]^{+}$calcd. for $\mathrm{C}_{22} \mathrm{H}_{26} \mathrm{FNO}_{3}, 372.1975$; found, 372.1984 .

tert-Butyl 4-(bis(4-fluorophenyl)(hydroxy)methyl)piperidine-1-carboxylate (33). To a cooled (ice bath) solution of 4-fluorophenylmagnesium bromide in THF (1 M, $8.75 \mathrm{ml})$ was added di-tert-butyl piperidine-1,4-dicarboxylate $(0.5 \mathrm{~g}, 2.0 \mathrm{mmol})$, dissolved in anhydrous THF (10 ml). The mixture was heated to $60{ }^{\circ} \mathrm{C}$ for $3 \mathrm{~h}$. After quenching with brine at $0{ }^{\circ} \mathrm{C}$ (ice bath) the mixture was concentrated to dryness. The crude product was taken up in water and extracted into ethyl acetate. The organic layer was dried $\left(\mathrm{MgSO}_{4}\right)$, filtered and concentrated to give a light yellow solid. Recrystallization from ethanol gave a white solid (0.38 g, 47\%). Mp: $152-152{ }^{\circ} \mathrm{C}$. ${ }^{1} \mathrm{H}$ NMR $\left(\mathrm{CDCl}_{3}, 300 \mathrm{MHz}\right): \delta 7.42-7.37$ (m, 2H, ph-2,6H), 7.01-6.96 (m, 2H, ph-3,5H), 4.15

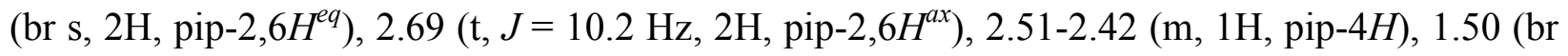
s, 2H, pip-3,5H $\left.H^{e q}\right), 1.42\left(\mathrm{~s}, 9 \mathrm{H}, \mathrm{C}\left(\mathrm{CH}_{3}\right)_{3}\right), 1.34-1.21\left(\mathrm{~m}, 2 \mathrm{H}, \mathrm{pip}-3,5 H^{a x}\right) .{ }^{13} \mathrm{C} \mathrm{NMR}\left(\mathrm{CDCl}_{3}, 125\right.$ $\mathrm{MHz}): \delta 161.7\left(\mathrm{~d},{ }^{1} J_{\mathrm{C}, \mathrm{F}}=244.5 \mathrm{~Hz}, \mathrm{ph}-4 C\right), 154.8(C=\mathrm{O}), 141.3(\mathrm{ph}-1 C), 127.6\left(\mathrm{~d},{ }^{3} J_{\mathrm{C}, \mathrm{F}}=7.8\right.$ $\mathrm{Hz}, \mathrm{ph}-2,6 C), 115.2\left(\mathrm{~d},{ }^{2} J_{\mathrm{C}, \mathrm{F}}=21.0 \mathrm{~Hz}, \mathrm{ph}-3,5 C\right), 79.6(\mathrm{COH}), 79.2\left(C\left(\mathrm{CH}_{3}\right)_{3}\right), 44.6($ pip-4C), 43.9 (pip-2,6C), $28.5\left(\mathrm{C}\left(\mathrm{CH}_{3}\right)_{3}\right), 26.5$ (pip-3,5C). ${ }^{19} \mathrm{~F}$ NMR (DMSO- $\left.d_{6}, 282 \mathrm{MHz}\right): \delta-117.77$ (ph-F). HRMS (m/z): $[\mathrm{M}+\mathrm{H}]^{+}$calcd. for $\mathrm{C}_{23} \mathrm{H}_{27} \mathrm{~F}_{2} \mathrm{NO}_{3}, 402.1881$; found, 402.1898 .

(4-Fluorophenyl)(phenyl)(piperidin-4-yl)methanol hydrochloride (34). To a solution of compound $32(0.56 \mathrm{~g}, 1.46 \mathrm{mmol})$ in THF $(1.0 \mathrm{ml})$ was added $\mathrm{HCl}$ in dioxane $(4 \mathrm{M}, 0.8 \mathrm{ml})$. The solution was stirred overnight at room temperature, while the product slowly crystallized as 
hydrochloric salt. The precipitate was filtered off and washed with THF and diethyl ether to give the product as a white solid $(0.32 \mathrm{~g}, 68 \%)$. Mp: $240-242{ }^{\circ} \mathrm{C} .{ }^{1} \mathrm{H}$ NMR (DMSO- $d_{6}, 500 \mathrm{MHz}$ ): $\delta$ 9.20/8.54 (br s/br s, $\left.1 \mathrm{H} / 1 \mathrm{H}, \mathrm{NH}_{2}{ }^{+}\right), 7.53-7.48(\mathrm{~m}, 4 \mathrm{H}, \mathrm{ph}-2,6 H, \mathrm{ph}-2,6 H), 7.28(\mathrm{t}, J=7.7 \mathrm{~Hz}$, 2H, ph-3,5H), 7.14 (t, $J=7.3 \mathrm{~Hz}, 2 \mathrm{H}, \mathrm{ph}-4 H), 7.09$ (t, $J=8.8 \mathrm{~Hz}, 2 \mathrm{H}, \mathrm{ph}-3,5 H), 5.66(\mathrm{CO} H)$, $3.19\left(\mathrm{~d}, J=12.0 \mathrm{~Hz}, 2 \mathrm{H}\right.$, pip-2,6 $\left.6 H^{e q}\right), 2.84$ (br s, 3H, pip-2,6 $H^{a x}$, pip- $4 H$ ), 1.64-1.56 (m, 2H, pip$\left.3,5 H^{a x}\right), 1.37\left(\mathrm{t}, J=10.5,2 \mathrm{H}, \mathrm{pip}-3,5 H^{e q}\right) .{ }^{13} \mathrm{C} \mathrm{NMR}\left(\mathrm{CDCl}_{3}, 125 \mathrm{MHz}\right): \delta 160.6\left(\mathrm{~d},{ }^{1} J_{\mathrm{C}, \mathrm{F}}=241.4\right.$ Hz, ph'-4C), 146.4 (ph-1C), 142.8 (ph'-1C), 128.0 (ph-3,5C), $127.8\left(\mathrm{~d},{ }^{3} J_{\mathrm{C}, \mathrm{F}}=7.9 \mathrm{~Hz}, \mathrm{ph}-2,6 C\right)$, $126.2(\mathrm{ph}-4 C), 125.7(\mathrm{ph}-2,6 C), 114.5\left(\mathrm{~d},{ }^{2} J_{\mathrm{C}, \mathrm{F}}=20.9 \mathrm{~Hz}, \mathrm{ph}-3,5 C\right), 78.2(\mathrm{COH}), 43.2$ (pip2,6C), 40.9 (pip-4C), 23.3 (pip-3,5C). ${ }^{19} \mathrm{~F}$ NMR (DMSO- $d_{6}, 282 \mathrm{MHz}$ ): $\delta-117.66(\mathrm{ph}-F)$. HRMS (m/z): $[\mathrm{M}+\mathrm{H}]^{+}$calcd. for $\mathrm{C}_{18} \mathrm{H}_{20} \mathrm{FNO}, 286.1607$; found, 286.1594 .

Bis(4-fluorophenyl)(piperidin-4-yl)methanol hydrochloride (35). Compound 33 (0.5 g, 1.2 mmol) was dissolved in THF $(2 \mathrm{ml}) . \mathrm{HCl}$ in dioxane $(4 \mathrm{M}, 0.6 \mathrm{ml})$ was added dropwise and the solution was stirred overnight at room temperature. During this time a white precipitate formed, which was filtered off, washed with DCM and diethyl ether and dried (0.24 g, 60\%). Mp: 244 ${ }^{\circ} \mathrm{C} .{ }^{1} \mathrm{H}$ NMR (DMSO- $\left.d_{6}, 500 \mathrm{MHz}\right): \delta 9.16 / 8.52\left(\mathrm{~d} / \mathrm{d}, J=9.7 / 9.7 \mathrm{~Hz}, 1 \mathrm{H} / 1 \mathrm{H}, \mathrm{NH}_{2}{ }^{+}\right), 7.51-7.49$ (m, 4H, ph-2,6H), $7.10(\mathrm{t}, J=8.8 \mathrm{~Hz}, 4 \mathrm{H}, \mathrm{ph}-3,5 H), 5.73(\mathrm{~s}, 1 \mathrm{H}, \mathrm{COH}), 3.19(\mathrm{~d}, J=12.0 \mathrm{~Hz}, 2 \mathrm{H}$,

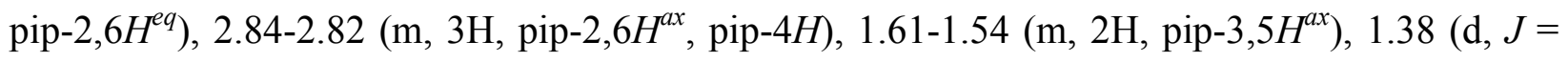
$\left.13.5 \mathrm{~Hz}, 2 \mathrm{H}, \mathrm{pip}-3,5 H^{e q}\right) .{ }^{13} \mathrm{C} \mathrm{NMR}\left(\mathrm{DMSO}-d_{6}, 125 \mathrm{MHz}\right): \delta 160.6\left(\mathrm{~d},{ }^{1} J_{\mathrm{C}, \mathrm{F}}=241.4 \mathrm{~Hz}, \mathrm{ph}-4 C\right)$, $142.6\left(\right.$ ph-1C), $127.8\left(\mathrm{~d},{ }^{3} J_{\mathrm{C}, \mathrm{F}}=7.8 \mathrm{~Hz}, \mathrm{ph}-2,6 C\right), 114.6\left(\mathrm{~d},{ }^{2} J_{\mathrm{C}, \mathrm{F}}=20.9 \mathrm{~Hz}, \mathrm{ph}-3,5 C\right), 78.0$ $(C O H), 43.2$ (pip-2,6C), 40.9 (pip-4C), 23.3 (pip-3,5C). ${ }^{19}$ F NMR (DMSO-d, $282 \mathrm{MHz}$ ): $\delta:-$ $117.47(\mathrm{ph}-F)$. HRMS (m/z): $[\mathrm{M}+\mathrm{H}]^{+}$calcd. for $\mathrm{C}_{18} \mathrm{H}_{19} \mathrm{~F}_{2} \mathrm{NO}, 304.1513$; found, 304.1516.

4-(4-((4-Fluorophenyl)(hydroxy)(phenyl)methyl)piperidin-1-yl)butyl acetate (38). Under inert atmosphere, compound $34(0.1 \mathrm{~g}, 0.31 \mathrm{mmol})$, 4-bromobutyl acetate $(0.09 \mathrm{ml}, 0.62 \mathrm{mmol})$ 
and potassium carbonate $(0.13 \mathrm{~g}, 0.93 \mathrm{mmol})$ were dissolved in anhydrous acetone $(5 \mathrm{ml})$. After heating at $60{ }^{\circ} \mathrm{C}$ for $3 \mathrm{~h}$ the mixture was stirred overnight at room temperature. The inorganic compounds were filtered off and the crude purified by column chromatography (DCM: methanol $=$ 100: $0 \rightarrow$ 95: 5). The product was isolated as colorless oil $(117 \mathrm{mg}, 94 \%) .{ }^{1} \mathrm{H}$ NMR (DMSO$\left.d_{6}, 600 \mathrm{MHz}\right): \delta 7.53-7.48(\mathrm{~m}, 4 \mathrm{H}, \mathrm{ph}-2,6 C$, ph'-2,6C), $7.27(\mathrm{t}, J=7.8 \mathrm{~Hz}, 2 \mathrm{H}, \mathrm{ph}-3,5 H), 7.13(\mathrm{t}$, $J=7.3 \mathrm{~Hz}, 1 \mathrm{H}, \mathrm{ph}-4 H), 7.07(\mathrm{~d}, J=8.9 \mathrm{~Hz}, 2 \mathrm{H}, \mathrm{ph}-3,5 H), 5.37(\mathrm{~s}, 1 \mathrm{H}, \mathrm{COH}), 3.98(\mathrm{t}, J=6.5$ Hz, 2H, but- $1 H_{2}$ ), 2.90 (br s, $2 \mathrm{H}$, pip-2,6H $H^{\mathrm{eq}}$ ), 2.50 (br s, $1 \mathrm{H}$, pip- $4 H$ ), 2.31 (br s, $2 \mathrm{H}$, but- $4 H_{2}$ ), $1.98\left(\mathrm{~m}, 5 \mathrm{H}, \mathrm{CH}_{3}\right.$, pip-2,6 $\left.H^{\mathrm{ax}}\right), 1.56-1.46$ (m, 6H, but-2,3H, pip-3,5H $\left.{ }^{\mathrm{eq}}\right), 1.24$ (br s, 2H, pip$\left.3,5 H^{\mathrm{ax}}\right) .{ }^{13} \mathrm{C}$ NMR (DMSO- $\left.d_{6}, 150 \mathrm{MHz}\right): \delta 170.5(C=\mathrm{O}), 160.5\left(\mathrm{~d},{ }^{1} J_{\mathrm{C}, \mathrm{F}}=240.8 \mathrm{~Hz}, \mathrm{ph}-4 C\right)$, 147.0 (ph-1C), 143.4 (ph'-1C), 127.9 (ph-3,5C), $127.8\left(\mathrm{~d},{ }^{3} J_{\mathrm{C}, \mathrm{F}}=7.7 \mathrm{~Hz}, \mathrm{ph}{ }^{\prime}-2,6 C\right), 126.0(\mathrm{ph}-$ 4C), 125.7 (ph-2,6C), $114.1\left(\mathrm{~d},{ }^{2} J_{\mathrm{C}, \mathrm{F}}=20.9 \mathrm{~Hz}, \mathrm{ph}-3,5 C\right), 78.2(C \mathrm{OH}), 63.8$ (but-1C), 57.3 (but4C), 53.4 (pip-2,6C), 43.3 (pip-4C), 26.0 (but-2C), 25.7 (pip-3,5C), 22.6 (but-3C), $20.8\left(C \mathrm{H}_{3}\right)$. ${ }^{19} \mathrm{~F}$ NMR (DMSO- $\left.d_{6}, 282 \mathrm{MHz}\right): \delta:-118.01(\mathrm{ph}-F)$. HRMS $(\mathrm{m} / \mathrm{z}):[\mathrm{M}+\mathrm{H}]^{+}$calcd. for $\mathrm{C}_{24} \mathrm{H}_{30} \mathrm{FNO}_{3}, 400.2288$; found, 400.2296.

4-(4-((4-Fluorophenyl)(hydroxy)(phenyl)methyl)piperidin-1-yl)butan-1-ol (39). Under inert atmosphere, compound $34(0.1 \mathrm{~g}, 0.31 \mathrm{mmol})$, 4-bromo-1-butanol $(0.1 \mathrm{~g}, 0.62 \mathrm{mmol})$ and potassium carbonate $(0.13 \mathrm{~g}, 0.93 \mathrm{mmol})$ were dissolved in anhydrous acetone $(5 \mathrm{ml})$. After heating at $60{ }^{\circ} \mathrm{C}$ for $3 \mathrm{~h}$ the mixture was stirred overnight at room temperature. The inorganic compounds were filtered off and the crude purified by column chromatography (DCM: methanol $=98: 2 \rightarrow 95: 5)$. The product was isolated as colorless oil $(87 \mathrm{mg}, 78 \%) .{ }^{1} \mathrm{H}$ NMR (DMSO- $d_{6}$, $600 \mathrm{MHz}): \delta 7.53-7.48(\mathrm{~m}, 4 \mathrm{H}, \mathrm{ph}-2,6 C, \mathrm{ph}-2,6 C), 7.29(\mathrm{t}, J=7.7 \mathrm{~Hz}, 2 \mathrm{H}, \mathrm{ph}-3,5 H), 7.15(\mathrm{t}, J$ $=7.3 \mathrm{~Hz}, 1 \mathrm{H}, \mathrm{ph}-4 H), 7.10(\mathrm{~d}, J=8.8 \mathrm{~Hz}, 2 \mathrm{H}, \mathrm{ph}-3,5 H), 5.56(\mathrm{~s}, 1 \mathrm{H}, \mathrm{COH}), 3.56(\mathrm{~s}, 1 \mathrm{H}$, $\mathrm{CH}_{2} \mathrm{OH}$ ), 3.39 (t, $J=6.3 \mathrm{~Hz}, 2 \mathrm{H}$, but- $1 H_{2}$ ), 3.33 (pip-2,6H , overlay with water), 3.19 (br s, $2 \mathrm{H}$, 


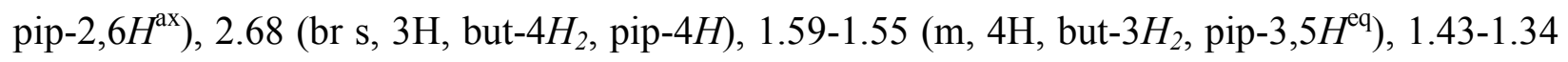
(m, 4H, but-2H , pip-3,5H $\left.H^{\mathrm{ax}}\right),{ }^{13} \mathrm{C}$ NMR (DMSO- $\left.d_{6}, 150 \mathrm{MHz}\right): \delta 160.6\left(\mathrm{~d},{ }^{1} J_{\mathrm{C}, \mathrm{F}}=241.1 \mathrm{~Hz}\right.$, ph'-4C), 146.6 (ph-1C), 143.0 (ph'-1C), 128.0 (ph-3,5C), 127.8 (d, $\left.{ }^{3} J_{\mathrm{C}, \mathrm{F}}=7.8 \mathrm{~Hz}, \mathrm{ph}{ }^{\prime}-2,6 C\right)$, $126.2(\mathrm{ph}-4 C), 125.7$ (ph-2,6C), $114.5\left(\mathrm{~d},{ }^{2} J_{\mathrm{C}, \mathrm{F}}=20.9 \mathrm{~Hz}, \mathrm{ph}-3,5 C\right), 78.1(C \mathrm{OH}), 60.3$ (but-1C), 56.8 (but-4C), 53.5 (pip-2,6C), 41.9 (pip-4C), 29.9 (but-2C), 24.7 (pip-3,5C), 21.6 (but-3C). ${ }^{19} \mathrm{~F}$ NMR (DMSO-d $\left.d_{6}, 282 \mathrm{MHz}\right): \delta-117.74(\mathrm{ph}-F)$. HRMS $(\mathrm{m} / \mathrm{z}):[\mathrm{M}+\mathrm{H}]^{+}$calcd. for $\mathrm{C}_{22} \mathrm{H}_{28} \mathrm{FNO}_{2}$, 358.2182; found, 358.2185.

\section{1-(3-(1,3-Dioxolan-2-yl)propyl)-4-((4-fluorophenyl)(phenyl)methylene)piperidine}

(41).

Under inert atmosphere, compound 36 (0.14 g, $0.53 \mathrm{mmol})$, 2-(3-chloropropyl)-1,3-dioxolane $(0.15 \mathrm{ml}, 1.06 \mathrm{mmol})$, potassium carbonate $(0.3 \mathrm{~g}, 2.1 \mathrm{mmol})$ and potassium iodide $(0.02 \mathrm{~g}, 0.1$ mmol) were dissolved in anhydrous acetonitrile $(10 \mathrm{ml})$. The mixture was heated overnight at 80 ${ }^{\circ} \mathrm{C}$. After cooling, the inorganic compounds were filtered off and the crude purified by column chromatography $(\mathrm{DCM}$ : methanol $=100: 0 \rightarrow 95: 5)$. The product was isolated as colorless oil $(50 \mathrm{mg}, 25 \%) .{ }^{1} \mathrm{H} \mathrm{NMR}\left(\mathrm{CDCl}_{3}, 600 \mathrm{MHz}\right): \delta 7.28(\mathrm{t}, J=7.6 \mathrm{~Hz}, 2 \mathrm{H}, \mathrm{ph}-3,5 H), 7.20(\mathrm{t}, J=7.3$ Hz, 1H, ph-4H), 7.10-7.06 (m, 4H, ph-2,6H, ph'-2,6H), $6.96(\mathrm{t}, J=8.6 \mathrm{~Hz}, 2 \mathrm{H}, \mathrm{ph}-3,5 H), 4.88$ $\left(\mathrm{t}, J=4.1 \mathrm{~Hz}, 1 \mathrm{H}\right.$, diox-CH), 3.96/3.84 (t/t, $J=6.9 / 6.9 \mathrm{~Hz}, 2 \mathrm{H} / 2 \mathrm{H}$, diox-4,5H $\mathrm{H}_{2}, 2.51$ (s, 4H, pip2,6H $), 2.40-2.39\left(\mathrm{~m}, 6 \mathrm{H}\right.$, prop- $1 H_{2}$, pip-3,5H2), $1.67\left(\mathrm{~m}, 4 \mathrm{H}\right.$, prop- $\left.2,3 H_{2}\right) .{ }^{13} \mathrm{C} \mathrm{NMR}\left(\mathrm{CDCl}_{3}\right.$, $150 \mathrm{MHz}): \delta 161.6\left(\mathrm{~d},{ }^{1} J_{\mathrm{C}, \mathrm{F}}=243.0 \mathrm{~Hz}, \mathrm{ph}-4 C\right), 142.4(\mathrm{ph}-1 C), 138.4\left(\mathrm{~d},{ }^{4} J_{\mathrm{C}, \mathrm{F}}=3.0 \mathrm{~Hz}, \mathrm{ph}{ }^{\prime}-\right.$ 1C), $135.9(C=\mathrm{C}), 134.9\left(\mathrm{C}=C^{\mathrm{pip}-4 C}\right), 131.5\left(\mathrm{~d},{ }^{3} J_{\mathrm{C}, \mathrm{F}}=7.5 \mathrm{~Hz}, \mathrm{ph}-2,6 C\right), 129.9(\mathrm{ph}-2,6 C), 128.2$ (ph-3,5C), 126.6 (ph-4C), 115.0 (d, $\left.{ }^{2} J_{\mathrm{C}, \mathrm{F}}=21.0 \mathrm{~Hz}, \mathrm{ph}-3,5 C\right), 104.5$ (diox- $\left.C \mathrm{H}\right), 65.0$ (diox4,5C), 58.3 (prop-1C), 55.2 (pip-2,6C), 31.9 (pip-3,5C), 31.6 (prop-3C), 21.4 (prop-2C). ${ }^{19} \mathrm{~F}$ NMR (DMSO-d $\left.d_{6}, 282 \mathrm{MHz}\right): \delta-116.74\left(\mathrm{ph}{ }^{\prime}-F\right)$. HRMS (m/z): [M+H] $]^{+}$calcd. for $\mathrm{C}_{24} \mathrm{H}_{28} \mathrm{FNO}_{2}$, 382.2182; found, 382.2175. 


\section{4-(4-((4-Fluorophenyl)(phenyl)methylene)piperidin-1-yl)butanal (42). Compound 41 (30} $\mathrm{mg}, 0.08 \mathrm{mmol})$ was dissolved in THF $(0.5 \mathrm{ml})$ and $\mathrm{HCl}(1 \mathrm{M} ; 1.5 \mathrm{ml})$, and the solution was stirred overnight at room temperature. The mixture was subsequently diluted with DCM and extracted with aqueous $\mathrm{NaOH}(2 \mathrm{M})$. The separated organic layer was dried $\left(\mathrm{MgSO}_{4}\right)$, filtered and concentrated under reduced pressure to yield the product as colorless oil $(20 \mathrm{mg}, 74 \%) .{ }^{1} \mathrm{H}$ $\operatorname{NMR}\left(\mathrm{CDCl}_{3}, 600 \mathrm{MHz}\right): \delta 9.71(\mathrm{CHO}), 7.28(\mathrm{t}, J=7.6 \mathrm{~Hz}, 2 \mathrm{H}, \mathrm{ph}-3,5 H), 7.20(\mathrm{t}, J=7.4 \mathrm{~Hz}$, 1H, ph-4H), 7.10-7.06 (m, 4H, ph-2,6H, ph'-2,6H), 6.96 (t, J=8.6 Hz, 2H, ph'-3,5H), 2.50 (br s,

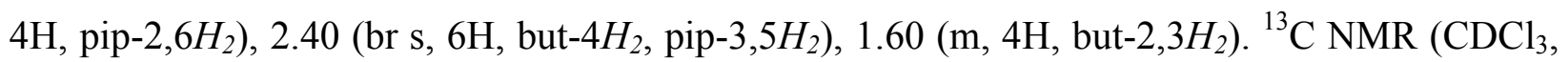
$150 \mathrm{MHz}): \delta 201.4(C \mathrm{HO}), 161.6\left(\mathrm{~d},{ }^{1} J_{\mathrm{C}, \mathrm{F}}=245.3 \mathrm{~Hz}, \mathrm{ph}{ }^{-4 C}\right), 142.4$ (ph-1C), $138.4(\mathrm{ph}-1 C)$, $136.1(C=\mathrm{C}), 134.8\left(\mathrm{C}=C^{\mathrm{pip}-4 C}\right), 131.4\left(\mathrm{~d},{ }^{3} J_{\mathrm{C}, \mathrm{F}}=7.8 \mathrm{~Hz}, \mathrm{ph}-2,6 C\right), 129.9(\mathrm{ph}-2,6 C), 128.2(\mathrm{ph}-$ 3,5C), $126.6(\mathrm{ph}-4 C), 115.0\left(\mathrm{~d},{ }^{2} J_{\mathrm{C}, \mathrm{F}}=21.1 \mathrm{~Hz}, \mathrm{ph}-3,5 C\right), 55.3$ (but-4C), 52.9 (pip-2,6C), 31.6 (but-2C), 30.6 (pip-3,5C), 22.1 (but-3C). ${ }^{19} \mathrm{~F}$ NMR (DMSO- $d_{6}, 282 \mathrm{MHz}$ ): $\delta-116.53$ (ph'-F). HRMS (m/z): [M+H] $]^{+}$calcd. for $\mathrm{C}_{22} \mathrm{H}_{24} \mathrm{FNO}, 338.1920$; found, 338.1912.

4-(4-((4-Fluorophenyl)(phenyl)methylene)piperidin-1-yl)butanoic acid (44). Compound 43 (0.3 $\mathrm{g}, 0.8 \mathrm{mmol})$ was dissolved in methanol $(1 \mathrm{ml})$ and THF $(1 \mathrm{ml})$. Aqueous potassium hydroxide $(2 \mathrm{M}, 2 \mathrm{ml})$ was added and the mixture was stirred for $3 \mathrm{~h}$ at room temperature. The organic solvents were subsequently removed under reduced pressure and the aqueous solution neutralized with hydrogen chloride solution. An oily product separating from the mixture was extracted into DCM. The dried and concentrated product was purified by column chromatography $(\mathrm{DCM}:$ methanol $=97: 3 \rightarrow 7: 3)$ and isolated as white solid $(0.1 \mathrm{~g}, 36 \%)$. Mp: 115-117 ${ }^{\circ} \mathrm{C} .{ }^{1} \mathrm{H}$ NMR (DMSO- $\left.d_{6}, 600 \mathrm{MHz}\right): \delta 7.32(\mathrm{t}, J=7.6 \mathrm{~Hz}, 2 \mathrm{H}, \mathrm{ph}-3,5 H), 7.23(\mathrm{t}, J=7.4$ Hz, 1H, ph-4H), 7.16-7.09 (m, 6H, ph-2,6H, ph'-2,3,5,6H), 2.60 (br s, 4H, pip-2,6H2), 2.27 (t, $J$ $=7.0 \mathrm{~Hz}, 2 \mathrm{H}$, but-4H $), 2.30(\mathrm{t}, J=5.5 \mathrm{~Hz}, 4 \mathrm{H}$, pip-3,5H $), 2.26\left(\mathrm{t}, J=7.1 \mathrm{~Hz}, 2 \mathrm{H}\right.$, but- $\left.2 H_{2}\right)$, 
1.72-1.67 (m, 2H, but-3H $\left.H_{2}\right) .{ }^{13} \mathrm{C}$ NMR (DMSO- $\left.d_{6}, 150 \mathrm{MHz}\right): \delta 174.4(\mathrm{C}=\mathrm{O}), 160.9\left(\mathrm{~d},{ }^{1} J_{\mathrm{C}, \mathrm{F}}=\right.$ 242.0, ph'-4C), 141.8 (ph-1C), $138.2\left(\mathrm{~d},{ }^{4} J_{\mathrm{C}, \mathrm{F}}=3.2 \mathrm{~Hz}, \mathrm{ph}-1 C\right), 134.7(C=\mathrm{C}), 134.5\left(\mathrm{C}=C^{\mathrm{pip}-4 C}\right)$, $131.3\left(\mathrm{~d},{ }^{3} J_{\mathrm{C}, \mathrm{F}}=8.0 \mathrm{~Hz}, \mathrm{ph}-2,6 C\right), 129.4$ (ph-3,5C), 128.3 (ph-2,6C), 126.7 (ph-4C), $115.1(\mathrm{~d}$, ${ }^{2} J_{\mathrm{C}, \mathrm{F}}=21.2 \mathrm{~Hz}$, ph'-3,5C), 56.5 (but-4C), 54.0 (pip-2,6C), 32.1/21.3 (but-2,3C), 30.4 (pip-3,5C).

${ }^{19} \mathrm{~F}$ NMR (DMSO- $\left.d_{6}, 282 \mathrm{MHz}\right): \delta-116.35\left(\mathrm{ph}{ }^{\prime}-F\right)$. HRMS $(\mathrm{m} / \mathrm{z}):[\mathrm{M}+\mathrm{H}]^{+}$calcd. for $\mathrm{C}_{22} \mathrm{H}_{24} \mathrm{FNO}_{2}$, 354.1864; found, 354.1863.

Ethyl 4-(4-(4-fluorobenzoyl)piperidin-1-yl)butanoate (47). Under inert atmosphere, compound 37 (as $\mathrm{HCl}$ salt; $0.25 \mathrm{~g}, 1.0 \mathrm{mmol}$ ), 4-bromo-butyric acid ethyl ester (0.28 ml, 2.0 $\mathrm{mmol})$ and potassium carbonate $(0.57 \mathrm{~g}, 4.0 \mathrm{mmol})$ were dissolved in anhydrous acetone $(10 \mathrm{ml})$. After heating at $60{ }^{\circ} \mathrm{C}$ for $3 \mathrm{~h}$ the mixture was stirred overnight at room temperature. The inorganic compounds were filtered off and the crude purified by column chromatography (DCM: methanol $=100: 0 \rightarrow 96: 4)$. The product was isolated as colorless oil $(0.28 \mathrm{~g}, 88 \%)$. An analytical sample of the product was crystallized as $\mathrm{HCl}$ salt. $\mathrm{Mp}: 138-139{ }^{\circ} \mathrm{C} .{ }^{1} \mathrm{H}$ NMR (DMSO- $\left.d_{6}, 600 \mathrm{MHz}\right): \delta 10.67$ (br s, $1 \mathrm{H}, \mathrm{NH}^{+}$), 8.11-8.09 (dd, $\left.J=8.7 \mathrm{~Hz}, 2 \mathrm{H}, \mathrm{ph}-2,6 H\right), 7.40$ $(\mathrm{dd}, J=8.8 \mathrm{~Hz}, 2 \mathrm{H}, \mathrm{ph}-3,5 H), 4.08\left(\mathrm{q}, 2 \mathrm{H}, \mathrm{OCH}_{2} \mathrm{CH}_{3}\right), 3.72(\mathrm{~m}, 1 \mathrm{H}, \mathrm{pip}-4 H), 3.52(\mathrm{~d}, J=11.8$

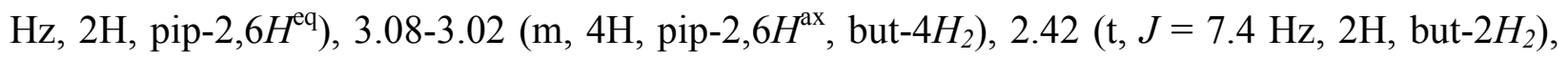
2.00-1.92 (m, 6H, pip-3,5H $\mathrm{H}_{2}$, but-3 $\left.\mathrm{H}_{2}\right), 1.20\left(\mathrm{t}, J=7.1 \mathrm{~Hz}, 3 \mathrm{H}, \mathrm{OCH}_{2} \mathrm{CH}_{3}\right) .{ }^{13} \mathrm{C}$ NMR (DMSO$\left.d_{6}, 150 \mathrm{MHz}\right): \delta 199.6\left(C=\mathrm{O}^{\text {ketone }}\right), 172.0\left(C=\mathrm{O}^{\text {ester }}\right), 165.2\left(\mathrm{~d},{ }^{1} J_{\mathrm{C}, \mathrm{F}}=250.7 \mathrm{~Hz}, \mathrm{ph}-4 C\right), 131.9(\mathrm{~d}$, $\left.{ }^{4} J_{\mathrm{C}, \mathrm{F}}=2.7, \mathrm{ph}-1 C\right), 131.4\left(\mathrm{~d},{ }^{3} J_{\mathrm{C}, \mathrm{F}}=9.3\right.$, ph-2,6C), $116.01\left(\mathrm{~d},{ }^{2} J_{\mathrm{C}, \mathrm{F}}=21.8, \mathrm{ph}-3,5 C\right), 60.1$ $\left(\mathrm{OCH}_{2} \mathrm{CH}_{3}\right.$ ), 55.2 (but-4C), 52.4 (pip-2,6C), 30.6 (but-2C), 25.6 (pip-3,5C), 18.7 (but-3C), 14.2 $\left(\mathrm{OCH}_{2} \mathrm{CH}_{3}\right) .{ }^{19} \mathrm{~F}$ NMR (DMSO- $\left.d_{6}, 282 \mathrm{MHz}\right): \delta-106.04(\mathrm{ph}-F) . \mathrm{HRMS}(\mathrm{m} / \mathrm{z}):[\mathrm{M}+\mathrm{H}]^{+}$calcd. for $\mathrm{C}_{18} \mathrm{H}_{24} \mathrm{FNO}_{2}, 322.1818$; found, 322.1813 . 
2-(2,2-Dimethyl-1,3-dioxolan-4-yl)ethyl 4-methylbenzenesulfonate (48). 2-(2,2-Dimethyl[1,3]dioxolan-4-yl)-ethanol (5 g, $34 \mathrm{mmol})$ was dissolved in DCM (100 ml). The solution was cooled (ice bath) and pyridine $(3 \mathrm{ml}, 37.4 \mathrm{mmol})$ and tosyl chloride $(7.1 \mathrm{~g}, 37.4 \mathrm{mmol})$ were subsequently added. The mixture was stirred overnight at room temperature. After dilution with DCM the reaction was quenched with water. The separated organic layer was washed with brine, dried $\left(\mathrm{MgSO}_{4}\right)$ and concentrated to dryness. The product was purified by column chromatography (DCM) giving a colorless oil $(2.8 \mathrm{~g}, 27.5 \%) .{ }^{1} \mathrm{H}$ NMR (DMSO- $\left.d_{6}, 600 \mathrm{MHz}\right): \delta$ $7.78(\mathrm{~d}, J=8.3 \mathrm{~Hz}, 2 \mathrm{H}, \mathrm{ph}-2,6 H), 7.35$ (d, $J=7.5 \mathrm{~Hz}, 2 \mathrm{H}, \mathrm{ph}-3,5 H), 4.19-4.09$ (m, 3H, diox$\mathrm{CH}_{2}$, diox-4H), 4.01/3.51 (t/t, $J=4.7 / 5.0 \mathrm{~Hz}, 1 \mathrm{H} / 1 \mathrm{H}$, diox $\left.-5 H_{2}\right), 2.45\left(\mathrm{~s}, 3 \mathrm{H}, \mathrm{ph}-\mathrm{CH}_{3}\right), 1.94-1.86$ $\left(\mathrm{m}, 2 \mathrm{H}, \mathrm{OCH}_{2}\right), 1.33 / 1.28\left(\mathrm{~s} / \mathrm{s}, 3 \mathrm{H} / 3 \mathrm{H}, \mathrm{C}\left(\mathrm{CH}_{3}\right)_{2}\right) .{ }^{13} \mathrm{C} \mathrm{NMR}\left(\mathrm{DMSO}-d_{6}, 150 \mathrm{MHz}\right): \delta 145.0(\mathrm{ph}-$ 4C), 133.0 (ph-1C), 130.1 (ph-3,5C), 128.1 (ph-2,6C), $109.2\left(C\left(\mathrm{CH}_{3}\right)_{2}\right), 72.4($ diox-4C), 69.2 (diox-5C), $67.5\left(\mathrm{O}-\mathrm{CH}_{2}\right), 33.2\left(\right.$ diox- $\left.\mathrm{CH}_{2}\right), 27.0 / 25.7\left(\mathrm{C}\left(\mathrm{CH}_{3}\right)_{2}\right), 21.8\left(\mathrm{ph}-\mathrm{CH}_{3}\right)$. HRMS (m/z): $[\mathrm{M}+\mathrm{H}]^{+}$calcd. for $\mathrm{C}_{14} \mathrm{H}_{20} \mathrm{O}_{5} \mathrm{~S}, 301.1110$; found, 301.1113 .

1-Benzhydryl-4-(2-(2,2-dimethyl-1,3-dioxolan-4-yl)ethyl)piperazine (49). TEA (1.24 ml, $9.3 \mathrm{mmol})$ was dissolved in dry acetonitrile $(100 \mathrm{ml})$. Under inert atmosphere, 1-benzhydrylpiperazine (2.3 g, $9.3 \mathrm{mmol})$ and compound 48 (2.8 g, $9.3 \mathrm{mmol})$ were subsequently added. The solution was stirred for $6 \mathrm{~h}$ at $70{ }^{\circ} \mathrm{C}$. After cooling it was concentrated to dryness and the product was purified by column chromatography (TEA; DCM: methanol $=100: 0 \rightarrow 95: 5)$ giving a yellow oil (1.36 g, 39\%). ${ }^{1} \mathrm{H}$ NMR (DMSO-d $\left.6,600 \mathrm{MHz}\right): \delta 7.40(\mathrm{~d}, J=7.7 \mathrm{~Hz}, 4 \mathrm{H}, \mathrm{ph}-2,6 H)$, $7.27(\mathrm{t}, J=7.7 \mathrm{~Hz}, 4 \mathrm{H}, \mathrm{ph}-3,5 H), 7.17(\mathrm{t}, J=7.4 \mathrm{~Hz}, 2 \mathrm{H}, \mathrm{ph}-4 H), 4.23$ (s, 1H, benzh.-CH), 4.02$3.98(\mathrm{~m}, 1 \mathrm{H}, \operatorname{diox}-4 H), 3.97 / 3.42\left(\mathrm{t} / \mathrm{t}, J=4.6 / 7.4 \mathrm{~Hz}, 1 \mathrm{H} / 1 \mathrm{H}, \operatorname{diox}-5 H_{2}\right), 2.49-2.24(\mathrm{~m}, 10 \mathrm{H}, \mathrm{prz}-$ 2,3,5,6 $\left.\mathrm{H}_{2}, \mathrm{NCH}_{2}\right), 1.63-1.59\left(\mathrm{~m}, 2 \mathrm{H}, \mathrm{OCH}_{2}\right), 1.28 / 1.23\left(\mathrm{~s} / \mathrm{s}, 3 \mathrm{H} / 3 \mathrm{H}, \mathrm{C}\left(\mathrm{CH}_{3}\right)_{2}\right) .{ }^{13} \mathrm{C} \mathrm{NMR}$ (DMSO- $d_{6}, 150$ MHz): $\delta 143.0(\mathrm{ph}-1 C), 128.5$ (ph-3,5C), 127.6 (ph-2,6C), 126.8 (ph-4C), 107.7 
$\left(C\left(\mathrm{CH}_{3}\right)_{2}\right), 75.3$ (benzh.- $\left.\mathrm{CH}\right), 74.2$ (diox-4C), 68.8 (diox-5C), $55.0\left(\mathrm{NCH}_{2}\right), 53.0$ (prz-2,6C), 51.6 (prz-3,5C), $30.6\left(\right.$ diox $\left.-\mathrm{CH}_{2}\right), 26.9 / 25.7\left(\mathrm{C}\left(\mathrm{CH}_{3}\right)_{2}\right)$. HRMS $(\mathrm{m} / \mathrm{z}):[\mathrm{M}+\mathrm{H}]^{+}$calcd. for $\mathrm{C}_{24} \mathrm{H}_{32} \mathrm{~N}_{2} \mathrm{O}_{2}, 381.2542$; found, 381.2550 .

4-(4-Benzhydrylpiperazin-1-yl)butane-1,2-diol (50). Compound 49 (1.36 g, 3.6 mmol) was dissolved in methanol $(13 \mathrm{ml})$. PTSA $(1.5 \mathrm{~g}, 7.9 \mathrm{mmol})$ was added and the solution was stirred for $4 \mathrm{~h}$ at $40{ }^{\circ} \mathrm{C}$. After cooling it was quenched with aqueous $\mathrm{NaOH}(2 \mathrm{~N})$. The organic solvent was removed under reduced pressure and the crude product extracted in DCM. Final purification was performed by column chromatography (TEA; DCM: methanol $=95: 5 \rightarrow 90: 10)$ giving the product as colorless oil $(1.1 \mathrm{~g}, 90 \%) .{ }^{1} \mathrm{H} \mathrm{NMR}\left(\mathrm{DMSO}-d_{6}, 600 \mathrm{MHz}\right): \delta 7.40(\mathrm{~d}, J=7.6 \mathrm{~Hz}, 4 \mathrm{H}$, ph-2,6H), 7.28 (t, $J=7.7 \mathrm{~Hz}, 4 \mathrm{H}, \mathrm{ph}-3,5 H), 7.17$ (t, $J=7.4 \mathrm{~Hz}, 2 \mathrm{H}, \mathrm{ph}-4 H), 4.64 / 4.60$ (br s/br s, 2H, OH/OH), $4.24(\mathrm{~s}, 1 \mathrm{H}$, benzh.- $\mathrm{CH}), 3.46-3.42(\mathrm{~m}, 1 \mathrm{H}, \mathrm{CHOH}), 3.28-3.25 / 3.21-3.16(\mathrm{~m} / \mathrm{m}$, $\left.1 \mathrm{H} / 1 \mathrm{H}, \mathrm{CH}_{2} \mathrm{OH}\right), 2.43-2.30\left(\mathrm{~m}, 10 \mathrm{H}, \mathrm{prz}-2,3,5,6 H_{2}, \mathrm{NCH}_{2}\right), 1.61-1.56 / 1.40-1.42(\mathrm{~m} / \mathrm{m}, 2 \mathrm{H}$, $\left.\mathrm{CH}_{2} \mathrm{CH}\right) .{ }^{13} \mathrm{C}$ NMR (DMSO- $\left.d_{6}, 150 \mathrm{MHz}\right): \delta 143.0$ (ph-1C), 128.55 (ph-3,5C), 127.57 (ph2,6C), 126.85 (ph-4C), 75.24 (benzh.- $C H), 70.46(C H O H), 65.9\left(\mathrm{CH}_{2} \mathrm{OH}\right), 55.0\left(\mathrm{NCH}_{2}\right), 53.0$ (prz-2,6C), 51.6 (prz-3,5C), $30.7\left(\mathrm{CH}_{2} \mathrm{CH}\right)$. HRMS (m/z): $[\mathrm{M}+\mathrm{H}]^{+}$calcd. for $\mathrm{C}_{21} \mathrm{H}_{28} \mathrm{~N}_{2} \mathrm{O}_{2}$, 341.2229; found, 341.2226.

4-(4-Benzhydrylpiperazin-1-yl)-2-hydroxybutyl acetate (51). Compound 50 (1.5 g, 1.5 mmol) was dissolved in DCM $(20 \mathrm{ml})$. TEA $(0.23 \mathrm{ml}, 1.65 \mathrm{mmol})$ and acetic anhydride $(0.14 \mathrm{ml}$, $1.5 \mathrm{mmol}$ ) were added under cooling (ice bath). The mixture was stirred $3 \mathrm{~h}$ at room temperature and subsequently quenched by adding aqueous $\mathrm{NaOH}(2 \mathrm{~N})$. The separated and washed (brine, water) organic layer was concentrated and purified by column chromatography (TEA; DCM: methanol $=97: 3)$ giving the product as colorless oil $(0.26 \mathrm{~g}, 46 \%) .{ }^{1} \mathrm{H}$ NMR (DMSO- $d_{6}, 600$ MHz): $\delta 7.41(\mathrm{~d}, J=7.5 \mathrm{~Hz}, 4 \mathrm{H}, \mathrm{ph}-2,6 H), 7.28(\mathrm{t}, J=7.7 \mathrm{~Hz}, 4 \mathrm{H}, \mathrm{ph}-3,5 H), 7.17(\mathrm{t}, J=7.3 \mathrm{~Hz}$, 
2H, ph-4H), 5.02 (br s, 1H, OH), 4.24 (s, 1H, benzh.-CH), $3.87(\mathrm{~d}, J=5.6 \mathrm{~Hz}, 2 \mathrm{H}$, $\left.\mathrm{CH}_{2} \mathrm{OCOCH}_{3}\right), 3.70-3.66(\mathrm{~m}, 1 \mathrm{H}, \mathrm{CHOH}), 2.52-2.09\left(\mathrm{~m}, 10 \mathrm{H}\right.$, prz-2,3,5,6H, $\left.\mathrm{NCH}_{2}\right), 1.99$ (s, $\left.3 \mathrm{H}, \mathrm{COCH}_{3}\right), 1.58-1.51 / 1.46-1.38\left(\mathrm{~m} / \mathrm{m}, 2 \mathrm{H}, \mathrm{CH}_{2} \mathrm{CH}\right) .{ }^{13} \mathrm{C}$ NMR (DMSO- $\left.d_{6}, 150 \mathrm{MHz}\right): \delta 170.4$ $(C=\mathrm{O}), 143.0$ (ph-1C), 128.5 (ph-3,5C), 127.6 (ph-2,6C), 126.9 (ph-4C), 75.2 (benzh.-CH), 68.0 $\left(\mathrm{CH}_{2} \mathrm{OCOCH}_{3}\right), 66.9(\mathrm{CHOH}), 54.5\left(\mathrm{NCH}_{2}\right), 53.0$ (prz-2,6C), 51.6 (prz-3,5C), $30.2\left(\mathrm{CH}_{2} \mathrm{CH}\right)$, $21.1\left(\mathrm{COCH}_{3}\right)$. HRMS (m/z): $[\mathrm{M}+\mathrm{H}]^{+}$calcd. for $\mathrm{C}_{23} \mathrm{H}_{30} \mathrm{~N}_{2} \mathrm{O}_{3}, 383.2335$; found, 383.2324.

4-(4-Benzhydrylpiperazin-1-yl)-2-((methylsulfonyl)oxy)butyl acetate (52). Compound 51 $(1.3 \mathrm{~g}, 3.4 \mathrm{mmol})$ was dissolved in DCM $(20 \mathrm{ml})$. TEA $(0.5 \mathrm{ml}, 3.7 \mathrm{mmol})$ and methanesulfonyl chloride $(0.3 \mathrm{ml}, 3.7 \mathrm{mmol})$ were added dropwise and under cooling (ice bath). The mixture was stirred $1 \mathrm{~h}$ at room temperature and subsequently quenched with aqueous $\mathrm{NaOH}(2 \mathrm{~N})$. The separated, dried $\left(\mathrm{MgSO}_{4}\right)$ and filtered organic layer was purified by column chromatography $($ TEA; DCM: methanol = 100: $0 \rightarrow 97: 3)$. The product was isolated as colorless oil $(1.1 \mathrm{~g}, 70 \%)$. ${ }^{1} \mathrm{H}$ NMR (DMSO- $\left.d_{6}, 600 \mathrm{MHz}\right): \delta 7.41(\mathrm{~d}, J=8.0 \mathrm{~Hz}, 4 \mathrm{H}, \mathrm{ph}-2,6 H), 7.28(\mathrm{t}, J=7.4,4 \mathrm{H}, \mathrm{ph}-$ 3,5H), $7.17(\mathrm{t}, J=7.2 \mathrm{~Hz}, 2 \mathrm{H}, \mathrm{ph}-4 H), 4.84-4.80(\mathrm{~m}, 1 \mathrm{H}, \mathrm{CHOMs}), 4.25 / 4.14$ (dd/dd, $J=$ 12.1/12.4 Hz, 1H/1H, $\left.\mathrm{CHCH}_{2} \mathrm{O}\right), 4.25$ (s, 1H, benzh.-CH), $3.18\left(\mathrm{~s}, 3 \mathrm{H}, \mathrm{SCH}_{3}\right), 2.50-2.10$ (m, $10 \mathrm{H}$, prz-2,3,5,6 $\left.\mathrm{H}_{2}, \mathrm{NCH}_{2}\right), 2.03\left(\mathrm{~s}, 3 \mathrm{H}, \mathrm{COCH}_{3}\right), 1.85-1.80\left(\mathrm{~m}, 2 \mathrm{H}, \mathrm{CH}_{2} \mathrm{CH}\right) .{ }^{13} \mathrm{C} \mathrm{NMR}$ $\left(\mathrm{DMSO}-d_{6}, 150 \mathrm{MHz}\right): \delta 170.1(C=\mathrm{O}), 143.0(\mathrm{ph}-1 C), 128.6(\mathrm{ph}-3,5 C), 127.6(\mathrm{ph}-2,6 C), 126.9$ (ph-4C), 78.5 (CHOMs), 75.3 (benzh.- $C \mathrm{H}), 64.7\left(\mathrm{CH}_{2} \mathrm{O}\right), 55.0\left(\mathrm{NCH}_{2}\right), 52.6$ (prz-2,6C), 51.6 (prz-3,5C), $37.7\left(\mathrm{SCH}_{3}\right), 27.9\left(\mathrm{CH}_{2} \mathrm{CH}\right), 20.6\left(\mathrm{COCH}_{3}\right)$. HRMS (m/z): $[\mathrm{M}+\mathrm{H}]^{+}$calcd. for $\mathrm{C}_{24} \mathrm{H}_{32} \mathrm{~N}_{2} \mathrm{O}_{5} \mathrm{~S}$, 461.2110; found, 461.2086.

(1-(2-(2,2-Dimethyl-1,3-dioxolan-4-yl)ethyl)piperidin-4-yl)diphenylmethanol (53). TEA (4 $\mathrm{ml}, 29.3 \mathrm{mmol})$ was dissolved in dry acetonitrile $(50 \mathrm{ml})$. Under inert atmosphere, diphenyl(piperidin-4-yl)methanol hydrochloride (8.64 g, $24.1 \mathrm{mmol})$ and compound 48 (7.2 g g, 
$24.1 \mathrm{mmol}$ ) were subsequently added. The solution was stirred for $6 \mathrm{~h}$ at $70{ }^{\circ} \mathrm{C}$. After cooling it was concentrated to dryness and the product was purified by column chromatography (TEA; DCM: methanol $=100: 0 \rightarrow 95: 5)$ giving a light yellow oil $(1.5 \mathrm{~g}, 16 \%) .{ }^{1} \mathrm{H}$ NMR $\left(\mathrm{DMSO}-d_{6}\right.$, $600 \mathrm{MHz}): \delta 7.50(\mathrm{~d}, J=8.2 \mathrm{~Hz}, 4 \mathrm{H}, \mathrm{ph}-2,6 H), 7.25(\mathrm{t}, J=7.7 \mathrm{~Hz}, 4 \mathrm{H}, \mathrm{ph}-3,5 H), 7.11(\mathrm{t}, J=7.3$ $\mathrm{Hz}, 2 \mathrm{H}, \mathrm{ph}-4 H), 5.23(\mathrm{~s}, 1 \mathrm{H}, \mathrm{OH}), 4.01-3.95(\mathrm{~m}, 2 \mathrm{H}, \operatorname{diox}-4 H$, diox $-5 H), 3.42(\mathrm{t}, J=7.2 \mathrm{~Hz}, 1 \mathrm{H}$, diox-5 $H_{2}$ ), 2.82 (br s, 2H, pip-2,6H $\left.H^{e q}\right), 2.45(\mathrm{t}, J=11.5 \mathrm{~Hz}, 1 \mathrm{H}, \mathrm{pip}-4 H), 2.28 / 2.24$ (br s/br s, $2 \mathrm{H}$, $\left.\mathrm{NCH}_{2}\right), 1.86-1.77\left(\mathrm{~m}, 2 \mathrm{H}\right.$, pip-2,6H $\left.{ }^{a x}\right), 1.62-1.60\left(\mathrm{~m}, 2 \mathrm{H}, \mathrm{CH}_{2} \mathrm{CH}\right), 1.47-1.43$ (m, 2H, pip$\left.3,5 H^{\mathrm{eq}}\right), 1.28 / 1.23\left(\mathrm{~s} / \mathrm{s}, 3 \mathrm{H} / 3 \mathrm{H}, \mathrm{C}\left(\mathrm{CH}_{3}\right)_{2}\right), 1.22\left(\right.$ br s, $2 \mathrm{H}$, pip-3,5 $\left.H^{\mathrm{ax}}\right) .{ }^{13} \mathrm{C}$ NMR $\left(\mathrm{DMSO}-d_{6}, 150\right.$ MHz): $\delta 147.4(\mathrm{ph}-1 C), 127.8(\mathrm{ph}-3-5 C), 125.6(\mathrm{ph}-2,6 C), 107.7\left(C\left(\mathrm{CH}_{3}\right)_{2}\right), 78.4(C \mathrm{OH}), 75.0$ (diox-4C), 68.3 (diox-5C), $66.1\left(\mathrm{NCH}_{2}\right), 53.6$ (pip-2,6C), 43.6 (pip-4C), 35.2 (diox- $\left.C \mathrm{H}_{2}\right)$, 26.9/25.8 $\left.\left(\mathrm{C}_{(\mathrm{CH}}\right)_{2}\right), 25.9$ (pip-3,5C). HRMS (m/z): $[\mathrm{M}+\mathrm{H}]^{+}$calcd. for $\mathrm{C}_{25} \mathrm{H}_{33} \mathrm{NO}_{3}, 396.2539$; found, 396.2533 .

4-(4-(Hydroxydiphenylmethyl)piperidin-1-yl)butane-1,2-diol (54). Compound 53 (1.5 g, $3.9 \mathrm{mmol})$ was dissolved in methanol $(15 \mathrm{ml})$. PTSA $(1.1 \mathrm{~g}, 5.8 \mathrm{mmol})$ was added and the solution was stirred for $4 \mathrm{~h}$ at $40^{\circ} \mathrm{C}$. After cooling it was quenched with aqueous $\mathrm{NaOH}(2 \mathrm{~N})$. The organic solvent was removed under reduced pressure and the crude product extracted in DCM. Final purification was performed by column chromatography $($ TEA; DCM: methanol = 95: $5 \rightarrow$ 90: 10) giving the product as colorless oil (1.35 g, quantitative conversion). ${ }^{1} \mathrm{H}$ NMR (DMSO- $\left.d_{6}, 300 \mathrm{MHz}\right): \delta 7.48(\mathrm{~d}, J=7.6 \mathrm{~Hz}, 4 \mathrm{H}, \mathrm{ph}-2,6 H), 7.24(\mathrm{t}, J=7.6 \mathrm{~Hz}, 4 \mathrm{H}, \mathrm{ph}-3,5 H)$, $7.10(\mathrm{t}, J=7.2 \mathrm{~Hz}, 2 \mathrm{H}, \mathrm{ph}-4 H), 5.26(\mathrm{~s}, 1 \mathrm{H}, \mathrm{OH}), 4.78($ br s, 2H, 2x OH), 3.46-3.42 (m, 1H, $\mathrm{CHOH}$ ), 3.29-3.15 (m, 2H, $\mathrm{CH}_{2} \mathrm{OH}$ ), 2.88 (br s, 2H, pip-2,6H $H^{e q}$, 2.49-2.34 (m, 3H, pip-4H, $\mathrm{NCH}_{2}$ ), 1.90 (br s, 2H, pip-2,6 $H^{a x}$ ), 1.56-1.35 (m, 4H, $\mathrm{CH}_{2} \mathrm{OH}$, pip-3,5H $\left.H^{\mathrm{eq}}\right), 1.25(\mathrm{~d}, J=12.2 \mathrm{~Hz}$, $2 \mathrm{H}$, pip-3,5H $\left.H^{\mathrm{ax}}\right) .{ }^{13} \mathrm{C}$ NMR (DMSO- $\left.d_{6}, 150 \mathrm{MHz}\right): \delta 147.3(\mathrm{ph}-1 C), 127.8(\mathrm{ph}-3-5 C), 125.8(\mathrm{ph}-$ 
2,6C), $78.5(\mathrm{COH}), 70.7(\mathrm{CHOH}), 65.9\left(\mathrm{CH}_{2} \mathrm{OH}\right), 55.3\left(\mathrm{NCH}_{2}\right), 53.6$ (pip-2,6C), 43.4 (pip-4C),

$30.4\left(\mathrm{CH}_{2} \mathrm{CH}(\mathrm{OH}) \mathrm{CH}_{2} \mathrm{OH}\right), 25.9$ (pip-3,5C). HRMS (m/z): $[\mathrm{M}+\mathrm{H}]^{+}$calcd. for $\mathrm{C}_{22} \mathrm{H}_{29} \mathrm{NO}_{3}$, 356.2226; found, 356.2216.

2-Hydroxy-4-(4-(hydroxydiphenylmethyl)piperidin-1-yl)butyl acetate (55). Compound 54 (1.35 g, $3.9 \mathrm{mmol})$ was dissolved in DCM $(50 \mathrm{ml})$. TEA $(0.6 \mathrm{ml}, 4.3 \mathrm{mmol})$ and acetic anhydride $(0.4 \mathrm{ml}, 3.9 \mathrm{mmol})$ were subsequently added and the solution was stirred for $3 \mathrm{~h}$ at room temperature. The reaction was quenched with aqueous $\mathrm{NaOH}(2 \mathrm{~N})$ and the organic layer was separated, dried $\left(\mathrm{MgSO}_{4}\right)$, filtered and concentrated under reduced pressure. Final purification was performed by column chromatography (TEA; DCM: methanol $=99: 1 \rightarrow 97: 3)$ giving the product as colorless oil $(0.6 \mathrm{~g}, 41 \%) .{ }^{1} \mathrm{H}$ NMR (DMSO- $\left.d_{6}, 600 \mathrm{MHz}\right): \delta 7.50(\mathrm{~d}, J=8.2 \mathrm{~Hz}, 4 \mathrm{H}$, ph-2,6H), 7.25 (t, $J=7.7 \mathrm{~Hz}, 4 \mathrm{H}, \mathrm{ph}-3,5 H), 7.11(\mathrm{t}, J=7.2 \mathrm{~Hz}, 2 \mathrm{H}, \mathrm{ph}-4 H), 5.26(\mathrm{~s}, 1 \mathrm{H}, \mathrm{COH})$, 4.76 (br s, $1 \mathrm{H}, \mathrm{CHOH}), 3.86\left(\mathrm{~d}, J=5.5 \mathrm{~Hz}, 2 \mathrm{H}, \mathrm{CH}_{2} \mathrm{OCOCH}_{3}\right), 3.71-3.67(\mathrm{~m}, 1 \mathrm{H}, \mathrm{CHOH}), 2.86$ $\left(\mathrm{t}, J=13.9 \mathrm{~Hz}, 2 \mathrm{H}\right.$, pip-2,6H $\left.H^{e q}\right), 2.47(\mathrm{t}, J=8.7 \mathrm{~Hz}, 1 \mathrm{H}, \mathrm{pip}-4 H), 2.39-2.31\left(\mathrm{~m}, 2 \mathrm{H}, \mathrm{NCH}_{2}\right), 2.00$ $\left(\mathrm{s}, 3 \mathrm{H}, \mathrm{COCH}_{3}\right), 1.86\left(\mathrm{t}, J=13.1 \mathrm{~Hz}, 2 \mathrm{H}, \mathrm{pip}-2,6 H^{a x}\right), 1.53-1.40\left(\mathrm{~m}, 4 \mathrm{H}, \mathrm{CH}_{2} \mathrm{CH}(\mathrm{OH})\right.$, pip$\left.3,5 H^{\mathrm{eq}}\right), 1.22\left(\mathrm{~d}, J=12.0 \mathrm{~Hz}, 2 \mathrm{H}, \mathrm{pip}-3,5 H^{\mathrm{ax}}\right) .{ }^{13} \mathrm{C}$ NMR (DMSO- $\left.d_{6}, 150 \mathrm{MHz}\right): \delta 170.4(C=\mathrm{O})$, 147.3 (ph-1C), 127.8 (ph-3-5C), $125.8(\mathrm{ph}-2,6 \mathrm{C}), 78.5(\mathrm{COH}), 68.0\left(\mathrm{CH}_{2} \mathrm{OCOCH}_{3}\right), 67.2$ $(C H O H), 55.0\left(\mathrm{NCH}_{2}\right), 53.7$ (pip-2,6C), 43.5 (pip-4C), $30.1\left(\mathrm{CH}_{2} \mathrm{CH}(\mathrm{OH}) \mathrm{CH}_{2}\right), 26.0$ (pip-3,5C), $20.8\left(\mathrm{COCH}_{3}\right)$. HRMS (m/z): $[\mathrm{M}+\mathrm{H}]^{+}$calcd. for $\mathrm{C}_{24} \mathrm{H}_{31} \mathrm{NO}_{4}, 398.2331$; found, 398.2331.

\section{4-(4-(Hydroxydiphenylmethyl)piperidin-1-yl)-2-((methylsulfonyl)oxy)butyl acetate (56).}

To a cooled (ice bath) solution of compound $55(0.32 \mathrm{~g}, 0.8 \mathrm{mmol})$ and TEA (0.12 ml, 0.88 mmol) in DCM $(10 \mathrm{ml})$ was added methanesulfonyl chloride $(0.07 \mathrm{ml}, 0.88 \mathrm{mmol})$ dropwise. The mixture was stirred $1 \mathrm{~h}$ at room temperature and subsequently quenched with brine. The separated, dried $\left(\mathrm{MgSO}_{4}\right)$ and filtered organic layer was purified by column chromatography 
$($ TEA; DCM: methanol = 99: $1 \rightarrow 98: 2)$. The product was isolated as colorless solid $(0.1 \mathrm{~g}$, 26\%). Mp: $78{ }^{\circ} \mathrm{C} .{ }^{1} \mathrm{H}$ NMR (DMSO- $\left.d_{6}, 600 \mathrm{MHz}\right): \delta 7.50(\mathrm{~d}, J=8.0 \mathrm{~Hz}, 4 \mathrm{H}, \mathrm{ph}-2,6 H), 7.25(\mathrm{t}, J$ $=7.3 \mathrm{~Hz}, 4 \mathrm{H}, \mathrm{ph}-3,5 H), 7.11(\mathrm{t}, J=7.3 \mathrm{~Hz}, 2 \mathrm{H}, \mathrm{ph}-4 H), 5.26(\mathrm{~s}, 1 \mathrm{H}, \mathrm{OH}), 4.83-4.79(\mathrm{~m}, 1 \mathrm{H}$, CHOMs), 4.27-4.12 (m, 2H, $\left.\mathrm{CH}_{2} \mathrm{OAc}\right), 3.19$ (s, 3H, $\left.\mathrm{SO}_{2} \mathrm{CH}_{3}\right), 2.84$ (t, $J=13.3 \mathrm{~Hz}, 2 \mathrm{H}$, pip2,6H $\left.H^{\mathrm{eq}}\right), 2.46(\mathrm{t}, J=11.9 \mathrm{~Hz}, 1 \mathrm{H}, \mathrm{pip}-4 H), 2.38-2.27\left(\mathrm{~m}, 2 \mathrm{H}, \mathrm{NCH}_{2}\right), 2.03\left(\mathrm{~s}, 3 \mathrm{H}, \mathrm{COCH}{ }_{3}\right), 1.90-$ $1.73\left(\mathrm{~m}, 4 \mathrm{H}, \mathrm{CH}_{2} \mathrm{CHF}\right.$, pip-2,6 $\left.H^{\mathrm{ax}}\right), 1.47-1.44\left(\mathrm{~m}, 2 \mathrm{H}, \mathrm{pip}-3,5 H^{\mathrm{eq}}\right), 1.21$ (d, $J=12.0 \mathrm{~Hz}, 2 \mathrm{H}$, pip$\left.3,5 H^{\mathrm{ax}}\right) .{ }^{13} \mathrm{C}$ NMR (DMSO- $\left.d_{6}, 150 \mathrm{MHz}\right): \delta 170.1(C=\mathrm{O}), 147.3(\mathrm{ph}-1 C), 127.8(\mathrm{ph}-3-5 C), 125.7$ (ph-2,6C), $78.5(\mathrm{COH}), 64.7\left(\mathrm{CH}_{2} \mathrm{OAc}\right), 53.8\left(\mathrm{NCH}_{2}\right), 53.1$ (pip-2,6C), 43.5 (pip-4C), 37.7 $\left(\mathrm{SO}_{2} \mathrm{CH}_{3}\right), 28.1\left(\mathrm{CH}_{2} \mathrm{CH}(\mathrm{OMs})\right), 26.0$ (pip-3,5C), $20.6\left(\mathrm{COCH}_{3}\right) . \mathrm{HRMS}(\mathrm{m} / \mathrm{z}):[\mathrm{M}+\mathrm{H}]^{+}$calcd. for $\mathrm{C}_{25} \mathrm{H}_{33} \mathrm{NO}_{6} \mathrm{~S}, 476.2107$; found, 476.2088 .

Ethyl 4-(4-(4-(phenylthio)benzoyl)piperidin-1-yl)butanoate (63). Under inert atmosphere, compound 60 (0.26 g, $0.8 \mathrm{mmol})$, 4-bromo-butyric acid ethyl ester (0.22 $\mathrm{ml}, 1.6 \mathrm{mmol})$ and potassium carbonate $(0.44 \mathrm{~g}, 3.2 \mathrm{mmol})$ were dissolved in anhydrous acetone $(10 \mathrm{ml})$. After heating at $60{ }^{\circ} \mathrm{C}$ for $3 \mathrm{~h}$ the mixture was stirred overnight at room temperature. The inorganic compounds were filtered off and the crude purified by column chromatography (DCM: methanol $=100: 0 \rightarrow 97: 3)$. The product was isolated as colorless oil $(0.26 \mathrm{~g}, 79 \%) .{ }^{1} \mathrm{H} \mathrm{NMR}\left(\mathrm{CDCl}_{3}, 600\right.$ MHz): $\delta 7.79(\mathrm{~d}, J=8.5 \mathrm{~Hz}, 2 \mathrm{H}, \mathrm{ph}-2,6 H), 7.50-7.49\left(\mathrm{~m}, 2 \mathrm{H}, \mathrm{ph}{ }^{\prime}-2,6 H\right), 7.40-7.39(\mathrm{~m}, 3 \mathrm{H}, \mathrm{ph}$ 3-5H), $7.20(\mathrm{~d}, J=8.5 \mathrm{~Hz}, 2 \mathrm{H}, \mathrm{ph}-3,5 H), 4.12$ (q, 2H, $\left.\mathrm{OCH}_{2} \mathrm{CH}_{3}\right), 3.18-3.15$ (m, 1H, pip-4H), $2.98\left(\mathrm{~d}, J=11.6 \mathrm{~Hz}, 2 \mathrm{H}\right.$, pip-2,6H $\left.H^{\mathrm{eq}}\right), 2.40(\mathrm{t}, J=7.2 \mathrm{~Hz}, 2 \mathrm{H}$, but-4H 2$), 2.33(\mathrm{t}, J=7.3 \mathrm{~Hz}, 2 \mathrm{H}$,

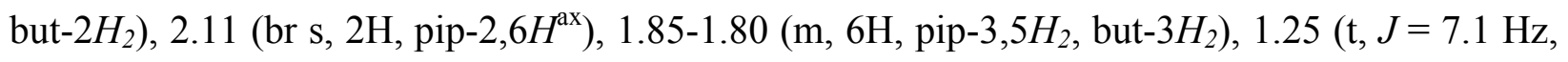
$\left.3 \mathrm{H}, \mathrm{OCH}_{2} \mathrm{CH}_{3}\right) .{ }^{13} \mathrm{C} \mathrm{NMR}\left(\mathrm{CDCl}_{3}, 150 \mathrm{MHz}\right): \delta 201.7\left(\mathrm{C}=\mathrm{O}^{\mathrm{ketone}}\right), 173.7\left(C=\mathrm{O}^{\text {ester }}\right), 144.9(\mathrm{ph}-$ 1C), 134.1 (ph-2,6C), 133.4 (ph-1C), 132.1 (ph'-1C), 129.91/129.89 (ph-3,5C, ph'-2,6C), 129.0 (ph'-3,5C), 127.6 (ph'-4C), $60.5\left(\mathrm{OCH}_{2} \mathrm{CH}_{3}\right), 57.8$ (but-4C), 53.1 (pip-2,6C), 43.4 (pip-4C), 32.4 
(but-2C), 28.6 (pip-3,5C), 22.2 (but-3C), $14.4\left(\mathrm{OCH}_{2} \mathrm{CH}_{3}\right)$. HRMS (m/z): $\left.\mathrm{M}+\mathrm{H}\right]^{+}$calcd. for $\mathrm{C}_{24} \mathrm{H}_{30} \mathrm{NO}_{3} \mathrm{~S}$, 412.1946; found, 412.1936.

\section{(4-(1-(4-Ethoxy-4-oxobutyl)piperidine-4-carbonyl)phenyl)diphenylsulfonium}

trifluoromethanesulfonate (64). To a solution of compound $63(0.3 \mathrm{~g}, 0.73 \mathrm{mmol})$ in chlorobenzene $(3 \mathrm{ml})$ were added trifluoromethanesulfonic acid $(65.0 \mu 1,0.73 \mathrm{mmol})$, diphenyliodonium triflate $(0.31 \mathrm{~g}, 0.73 \mathrm{mmol})$ and copper(II) benzoate hydrate $(11.5 \mathrm{mg}, 0.036$ mmol) and the mixture was heated at $125^{\circ} \mathrm{C}$ for $1 \mathrm{~h}$. After cooling, the resulting brown oil was washed with diethyl ether $(3 \times 20 \mathrm{ml})$. The product was purified by column chromatography $(\mathrm{DCM}:$ methanol = 10: $0 \rightarrow 9: 1)$. The isolated product was dissolved in DCM (5 ml) and washed with aqueous $\mathrm{NaOH}(2 \mathrm{M} ; 5 \mathrm{ml})$ and a saturated solution of sodium triflate $(5 \mathrm{ml})$. The organic phase was dried $\left(\mathrm{MgSO}_{4}\right)$, filtered and concentrated to give the product as a brown oil $(0.37 \mathrm{~g}$, 79\%). ${ }^{1} \mathrm{H}$ NMR (DMSO-d 6 , $\left.600 \mathrm{MHz}\right): \delta 8.27(\mathrm{~d}, J=8.5 \mathrm{~Hz}, 2 \mathrm{H}, \mathrm{ph}-2,6 H), 7.94(\mathrm{~d}, J=8.2 \mathrm{~Hz}$, 2H, ph-3,5H), 7.90-7.86 (m, 6H, ph'-2,4,6H), 7.81-7.78 (m, 4H, ph'-3,5H), 4.05 (q, 2H, $\mathrm{OCH}_{2} \mathrm{CH}_{3}$ ), 3.01/2.34/2.08/1.78 (v br s, $15 \mathrm{H}$, pip-2,3,5,6H , pip-4H, but-1-3H2), 1.19 (t, $J=7.1$ $\left.\mathrm{Hz}, 3 \mathrm{H}, \mathrm{OCH}_{2} \mathrm{CH}_{3}\right) .{ }^{13} \mathrm{C} \mathrm{NMR}\left(\mathrm{DMSO}-d_{6}, 150 \mathrm{MHz}\right): \delta 169.2\left(C=\mathrm{O}^{\text {ester }}\right), 134.6(\mathrm{ph}-1 C), 131.7$ (ph'-3,5C), 131.6 (ph-3,5C), 131.5 (ph-2,6C), 130.1 (ph'-2,6C), 124.9 (ph-4C), $120.7\left(\mathrm{q},{ }^{1} J_{\mathrm{C}, \mathrm{F}}=\right.$ $\left.320.4 \mathrm{~Hz}, \mathrm{CF}_{3}\right), 59.9\left(\mathrm{OCH}_{2} \mathrm{CH}_{3}\right), 14.2\left(\mathrm{OCH}_{2} \mathrm{CH}_{3}\right) .{ }^{19} \mathrm{~F}$ NMR (DMSO-d $\left.d_{6}, 282 \mathrm{MHz}\right): \delta-78.21$ $\left(\mathrm{CF}_{3}\right)$. HRMS (m/z): $[\mathrm{M}]^{+}$calcd. for $\mathrm{C}_{30} \mathrm{H}_{34} \mathrm{NO}_{3} \mathrm{~S}, 488.2259$; found, 488.2242 .

\section{1-(3-(1,3-Dioxolan-2-yl)propyl)-4-((4-((4-}

methoxyphenyl)thio)phenyl)(phenyl)methylene)piperidine (73). Under inert atmosphere, compound 68 (0.42 g, $1.1 \mathrm{mmol}), 2$-(3-chloropropyl)-1,3-dioxolane (0.3 ml, $2.2 \mathrm{mmol})$, potassium carbonate $(0.6 \mathrm{~g}, 4.4 \mathrm{mmol})$ and potassium iodide $(37 \mathrm{mg}, 0.22 \mathrm{mmol})$ were dissolved in anhydrous acetonitrile $(10 \mathrm{ml})$. After heating at $80{ }^{\circ} \mathrm{C}$ for $5 \mathrm{~h}$ the mixture was stirred overnight 
at room temperature. The inorganic compounds were filtered off and the crude purified by column chromatography $(\mathrm{DCM}:$ methanol $=98: 2 \rightarrow 8: 2)$. The product was isolated as colorless oil (0.13 g, 24\%). ${ }^{1} \mathrm{H}$ NMR (DMSO-d $\left.6,600 \mathrm{MHz}\right): \delta 7.42(\mathrm{~d}, J=8.6 \mathrm{~Hz}, 2 \mathrm{H}, \mathrm{ph}$ ',-2,6H), $7.30(\mathrm{t}$, $J=7.6 \mathrm{~Hz}, 2 \mathrm{H}, \mathrm{ph}-3,5 H), 7.21(\mathrm{t}, J=7.4 \mathrm{~Hz}, 1 \mathrm{H}, \mathrm{ph}-4 H), 7.06-7.04$ (m, 4H, ph-2,6H, ph'-2,6H), 7.01-6.99 (m, 4H, ph'-3,5H, ph'’-3,5H), $4.77(\mathrm{t}, J=4.5 \mathrm{~Hz}, 2 \mathrm{H}, \operatorname{diox}-\mathrm{CH}), 3.85 / 3.74(\mathrm{t} / \mathrm{t}, J=$ 6.9/6.9 Hz, 2H/2H, diox-4,5H $)$, $3.75\left(\mathrm{~s}, 3 \mathrm{H}, \mathrm{OCH}_{3}\right), 2.39-2.23$ (m, 10H, pip-2,3,5,6H , prop$\left.1 H_{2}\right), 1.56-1.50\left(\mathrm{~m}, 4 \mathrm{H}\right.$, but-2,3H $\left.H_{2}\right){ }^{13} \mathrm{C}$ NMR (DMSO-d $\left.6,150 \mathrm{MHz}\right): \delta 159.8(\mathrm{ph}$ '’-4C), 141.9 (ph-1C), 139.7 (ph'-1C), 136.0 (ph'-4C, $\left.C=\mathrm{C}^{\mathrm{pip}}\right), 135.6\left(\mathrm{ph}\right.$ '’-2,6C), $134.2\left(\mathrm{C}=C^{\mathrm{pip}}\right), 130.3$ (ph'3,5C), 129.4 (ph-2,6C), 128.2 (ph-3,5C), 127.2 (ph'-2,6C), 126.5 (ph-4C), 122.7 (ph'’-1C), 115.4 (ph'”-3,5C), 103.5 (diox- $C H$ ), 64.2 (diox-4,5C), 57.2 (prop-1C), $55.3\left(\mathrm{OCH}_{3}\right), 54.4$ (pip2,6C), 31.2 (pip-3,5C), 30.6 (prop-3C), 21.1 (prop-2C). HRMS (m/z): $[\mathrm{M}+\mathrm{H}]^{+}$calcd. for $\mathrm{C}_{31} \mathrm{H}_{35} \mathrm{NO}_{3} \mathrm{~S}, 502.2416$; found, 502.2412.

\section{(4-((1-(3-(1,3-Dioxolan-2-yl)propyl)piperidin-4-ylidene)(phenyl)methyl)phenyl)bis(4-}

methoxyphenyl)sulfonium trifluoromethanesulfonate (74). To a solution of compound 73 $(120 \mathrm{mg}, 0.24 \mathrm{mmol})$ in chlorobenzene $(1.0 \mathrm{ml})$ were added trifluoromethanesulfonic acid $(21.5$ $\mu 1,0.24 \mathrm{mmol}$ ), compound 75 (120 mg, $0.24 \mathrm{mmol}$ ) and copper(II) benzoate (4 mg, $0.01 \mathrm{mmol})$ and the mixture was heated at $125^{\circ} \mathrm{C}$ for $1 \mathrm{~h}$. After cooling, the resulting brown oil was washed with diethyl ether $(3 \times 5 \mathrm{ml})$. The product was purified by column chromatography (DCM: methanol $=10: 0 \rightarrow 9: 1)$. The isolated product was dissolved in DCM $(5 \mathrm{ml})$ and washed with aqueous $\mathrm{NaOH}(2 \mathrm{M} ; 5 \mathrm{ml})$ and a saturated solution of sodium triflate $(5 \mathrm{ml})$. The organic phase was dried $\left(\mathrm{MgSO}_{4}\right)$, filtered and concentrated to give the product as a light yellow oil (50 mg, 28\%). ${ }^{1} \mathrm{H}$ NMR (DMSO-d $\left.6,600 \mathrm{MHz}\right): \delta 7.67(\mathrm{~d}, J=8.9 \mathrm{~Hz}, 2 \mathrm{H}, \mathrm{ph}-2,6 H), 7.49(\mathrm{~d}, J=8.3 \mathrm{~Hz}$, 2H, ph'-2,6H), 7.39 (d, $J=8.4 \mathrm{~Hz}, 2 \mathrm{H}, \mathrm{ph} '-3,5 H), 7.30$ (t, $J=7.5 \mathrm{~Hz}, 2 \mathrm{H}, \mathrm{ph}$ '’-3,5H), 7.23 (t, $J$ 
$=7.4 \mathrm{~Hz}, 1 \mathrm{H}, \mathrm{ph}$ '-4H), 7.17 (d, $J=8.9 \mathrm{~Hz}, 4 \mathrm{H}, \mathrm{ph}-3,5 H), 7.07(\mathrm{~d}, J=7.7 \mathrm{~Hz}, 2 \mathrm{H}, \mathrm{ph}$ ' $-2,6 H)$, $4.87(\mathrm{t}, J=4.3 \mathrm{~Hz}, 2 \mathrm{H}$, diox-CH), 3.95/3.84 (t/t, $J=6.9 / 6.9 \mathrm{~Hz}, 2 \mathrm{H} / 2 \mathrm{H}$, diox-4,5H2), $3.90(\mathrm{~s}, 6 \mathrm{H}$, $\left.\mathrm{OCH}_{3}\right), 2.53-2.51$ (m, 4H, pip-2,6H2), 2.43-2.40 (m, 2H, prop- $\left.1 H_{2}\right), 2.37$ (s, 4H, pip-3,5H2), 1.68-1.60 (m, 4H, prop-2,3H2). ${ }^{13} \mathrm{C}$ NMR (DMSO- $\left.d_{6}, 150 \mathrm{MHz}\right): \delta 164.6(\mathrm{ph}-4 C), 148.7$ (ph'-

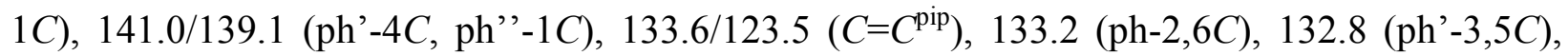
130.0 (ph'-2,6C), 129.9 (ph'”-2,6C), 128.6 (ph"-3,5C), $127.2(\mathrm{ph} "-4 C), 121.0$ (q, ${ }^{1} J_{\mathrm{C}, \mathrm{F}}=318.0$ $\mathrm{Hz}, \mathrm{CF}_{3}$ ), 117.3 (ph-3,5C), 114.4 (ph-1C), 104.4 (diox- $C H$ ), 65.0 (diox-4,5C), 58.1 (prop-1C), $56.2\left(\mathrm{OCH}_{3}\right), 55.0$ (pip-2,6C), 31.9 (pip-3,5C), 31.7 (prop-3C), 21.4 (prop-2C). ${ }^{19} \mathrm{~F}$ NMR (DMSO- $\left.d_{6}, 282 \mathrm{MHz}\right): \delta-78.05\left(\mathrm{CF}_{3}\right)$. HRMS (m/z): [M] $]^{+}$calcd. for $\mathrm{C}_{38} \mathrm{H}_{42} \mathrm{NO}_{4} \mathrm{~S}, 608.2662$; found, 608.2683 .

Radiochemistry - Equipment. All labeling reactions were performed manually using $\left[{ }^{18} \mathrm{~F}\right]$ fluoride in $\left[{ }^{18} \mathrm{O}\right] \mathrm{H}_{2} \mathrm{O}$. Radio-HPLC was performed with an Agilent $1200 \mathrm{HPLC}$ system equipped with a 1200 Series Diode Array Detector and a GABI Star NaI(Tl) scintillation detector. The system was used for purification as well as characterization of radiotracers.

General procedure for preparation of compounds $\left[{ }^{18} \mathbf{F}\right] 23$ and $\left[{ }^{18} \mathbf{F}\right] 27 \cdot\left[{ }^{18} \mathrm{~F}\right]$ Fluoride in water was trapped on a Sep-Pak ${ }^{\circledR}$ QMA cartridge and released with $0.5 \mathrm{ml}$ of a solution of Kryptofix $222(30 \mathrm{mM})$ and potassium carbonate $(7.5 \mathrm{mM})$ dissolved in acetonitrile: water $(85$ : 15). After removing the solvent by heating at $90{ }^{\circ} \mathrm{C}$ under a stream of nitrogen, acetonitrile $(0.5$ ml) was added, and the distillation was continued at $90{ }^{\circ} \mathrm{C}$. This procedure was repeated, the vial was subsequently capped and the mixture was allowed to come to room temperature. The respective mesylate precursor (4 $\mathrm{mg}$ in $0.2 \mathrm{ml}$ anhydrous DMSO) was added and the reaction was stirred at $90{ }^{\circ} \mathrm{C}$ for $20 \mathrm{~min}$. After quenching with $0.8 \mathrm{ml}$ water the crude mixture was purified by HPLC using a Chromolith ${ }^{\circledR}$ SemiPrep RP18-e column $(100 \times 10 \mathrm{~mm})$ at room 
temperature. The mobile phase consisted of water and methanol (each containing $0.5 \%$ TFA). Gradient elution starting with 30\% methanol content that was increased to $70 \%$ in 9 min allowed for isolation of the radioactive product. The obtained solution was diluted with $20 \mathrm{ml}$ water, trapped on a Sep-Pak ${ }^{\circledR}$ SPE C-18 light cartridge, and the radiolabeled product was released with $0.5 \mathrm{ml}$ ethanol. After having reduced the volume to $100 \mu \mathrm{l}$ under a stream of nitrogen, the solution was diluted with saline to give a final ethanol concentration of $5 \%$ and sterilized by filtration.

$\left[{ }^{18} \mathbf{F}\right] 4-(4-B e n z h y d r y l p i p e r a z i n-1-y l)-2-f l u o r o b u t y l ~ a c e t a t e ~\left(\left[{ }^{18} \mathbf{F}\right] 23\right)$. Starting with $1.2 \mathrm{GBq}$ fluoride-18 and using precursor 52, the synthetic procedure afforded $182 \mathrm{MBq}$ of formulated $\left[{ }^{18} \mathrm{~F}\right] 23$ (13\% overall RCY). Quality control was performed on a Chromolith ${ }^{\circledR}$ Performance RP18-e column $(100 \times 4.6 \mathrm{~mm})$ using water and methanol (each containing $0.5 \%$ TFA; gradient elution at a flow rate of $3 \mathrm{ml} / \mathrm{min}$ starting with $30 \%$ methanol content that was increased to $90 \%$ in $9 \mathrm{~min}$ ). The radiochemical purity was $100 \%$ and the identity of the radiochemical product was confirmed by co-elution with the non-radioactive analogue ( $\mathrm{RT}=4.6 \mathrm{~min})$.

$\left[{ }^{18} \mathrm{~F}\right] 2-F l u o r o-4-(4-(h y d r o x y d i p h e n y l m e t h y l) p i p e r i d i n-1-y l) b u t y l ~ a c e t a t e \quad\left(\left[{ }^{18} \mathrm{~F}\right] 27\right)$. Starting with $1.0 \mathrm{GBq}$ fluoride-18 and using precursor 56, the synthetic procedure afforded 105 $\mathrm{MBq}$ of formulated $\left[{ }^{18} \mathrm{~F}\right] 27$ (19\% overall RCY). Quality control was performed on a Chromolith $^{\circledR}$ Performance RP18-e column $(100 \times 4.6 \mathrm{~mm})$ using water and methanol (each containing $0.5 \%$ TFA; gradient elution at a flow rate of $3 \mathrm{ml} / \mathrm{min}$ starting with $30 \%$ methanol content that was increased to $90 \%$ in $9 \mathrm{~min}$ ). The radiochemical purity was $100 \%$ and the identity of the radiochemical product was confirmed by co-elution with the non-radioactive analogue $(\mathrm{RT}=4.5 \mathrm{~min})$. 


\section{$\left[{ }^{18}\right.$ F]4-(4-((4-Fluorophenyl)(hydroxy)(phenyl)methyl)piperidin-1-yl)butyl}

acetate

$\left(\left[{ }^{18} \mathbf{F}\right] 38\right) .\left[{ }^{18} \mathrm{~F}\right]$ Fluoride in water $(1.2 \mathrm{GBq})$ was trapped on a Sep-Pak ${ }^{\circledR}$ QMA cartridge, released with $0.5 \mathrm{ml}$ of a solution of Kryptofix $222(30 \mathrm{mM})$ and potassium hydrogen carbonate $(30 \mathrm{mM})$ dissolved in acetonitrile: water (85: 15). After removing the solvent by heating at $90{ }^{\circ} \mathrm{C}$ under a stream of nitrogen, acetonitrile $(0.5 \mathrm{ml})$ was added, and the distillation was continued at $90{ }^{\circ} \mathrm{C}$. This procedure was repeated and the reaction vial was subsequently capped. Compound 62 (4 mg) dissolved in DMSO $(0.5 \mathrm{ml})$ was subsequently added and the mixture was stirred at $110{ }^{\circ} \mathrm{C}$ for $15 \mathrm{~min}$. The reaction was quenched and further diluted with water to a volume of $20 \mathrm{ml}$. This solution was filtered over a Sep-Pak ${ }^{\circledR}$ SPE C-18 light cartridge. The radiolabeled product was released with acetonitrile $(1 \mathrm{ml})$, which was subsequently evaporated under a stream of nitrogen. The dry crude was taken up in THF $(100 \mu 1)$. Phenylmagnesium bromide $(100 \mu \mathrm{l}$ of a $1 \mathrm{M}$ solution in THF) was added and the solution reacted at $90{ }^{\circ} \mathrm{C}$ for $30 \mathrm{~min}$. After cooling, the reaction was quenched with formate buffer $(\mathrm{pH} 3 ; 1.8 \mathrm{ml})$ and purified by HPLC using a Chromolith $^{\circledR}$ SemiPrep RP18-e column $(100 \times 10 \mathrm{~mm})$ at room temperature. The mobile phase consisted of water and methanol (each containing $0.5 \%$ TFA). Gradient elution starting with $10 \%$ methanol content that was increased to $55 \%$ in 12 min and further to $90 \%$ in 3 min allowed

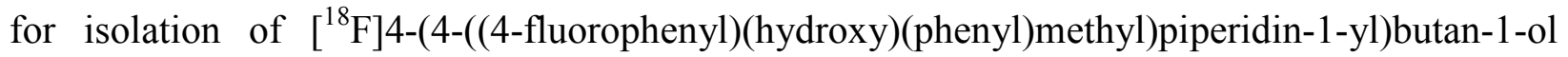
$\left(\left[{ }^{18} \mathrm{~F}\right] 39\right)$. The obtained solution was diluted with water to a final volume of $20 \mathrm{ml}$, trapped on a Sep-Pak ${ }^{\circledR}$ SPE C-18 light cartridge, and the radiolabeled product was released with acetonitrile (0.5 ml). TEA $(50 \mu \mathrm{l})$ and acetyl chloride $(25 \mu \mathrm{l})$ were added and the solution was stirred for 15 min at ambient temperature. The reaction was quenched with water $(1.4 \mathrm{ml})$ and the product was purified by HPLC using the same gradient that was used for purification of $\left[{ }^{18} \mathrm{~F}\right] 39$. The obtained solution was diluted with water to a final volume of $20 \mathrm{ml}$, trapped on a Sep-Pak ${ }^{\circledR}$ SPE C-18 
light cartridge, and the radiolabeled product was released with $0.5 \mathrm{ml}$ ethanol. After having reduced the volume to $100 \mu \mathrm{l}$ under a stream of nitrogen, the solution was diluted with saline to give a final ethanol concentration of $5 \%$ and sterilized by filtration. Starting with $3.0 \mathrm{GBq}$ fluoride-18, the three-step synthetic procedure afforded $13.4 \mathrm{MBq}$ of formulated $\left[{ }^{18} \mathrm{~F}\right] \mathbf{3 8}(6 \%$ overall RCY). Quality control was performed on a Chromolith ${ }^{\circledR}$ Performance RP18-e column $(100 \times 4.6 \mathrm{~mm})$ using water and methanol (each containing $0.5 \%$ TFA; gradient elution at a flow rate of $3 \mathrm{ml} / \mathrm{min}$ starting with $10 \%$ methanol content that was increased to $55 \%$ in 12 min and further to $90 \%$ in $3 \mathrm{~min}$ ). The radiochemical purity was $98 \%$ and the identity of the radiochemical product was confirmed by co-elution with the non-radioactive analogue $(\mathrm{RT}=9.5$ $\min )$.

\section{$\left[{ }^{18} \mathrm{~F}\right] 4-(4-((4-f l u o r o p h e n y l)(h y d r o x y)(p h e n y l) m e t h y l) p i p e r i d i n-1-y l) b u t a n-1-o l ~\left(\left[{ }^{18} \mathrm{~F}\right] 39\right)$.}

The compound was synthesized as described in the recipe for $\left[{ }^{18} \mathrm{~F}\right] 38$. After HPLC purification, the obtained solution was diluted with water to a final volume of $20 \mathrm{ml}$, trapped on a Sep-Pak ${ }^{\circledR}$ SPE C-18 light cartridge, and the radiolabeled product was released with $0.5 \mathrm{ml}$ ethanol. After having reduced the volume to $100 \mu 1$ under a stream of nitrogen, the solution was diluted with saline to give a final ethanol concentration of $5 \%$ and sterilized by filtration. The synthetic procedure afforded 28.4 MBq of formulated $\left[{ }^{18} \mathbf{F}\right] 39$ (6\% overall RCY). Quality control was performed on a Chromolith ${ }^{\circledR}$ Performance RP18-e column $(100 \times 4.6 \mathrm{~mm})$ using water and methanol (each containing $0.5 \%$ TFA; gradient elution at a flow rate of $3 \mathrm{ml} / \mathrm{min}$ starting with $10 \%$ methanol content that was increased to $55 \%$ in $12 \mathrm{~min}$ and further to $90 \%$ in $3 \mathrm{~min}$ ). The radiochemical purity was $100 \%$ and the identity of the radiochemical product was confirmed by co-elution with the non-radioactive analogue $(\mathrm{RT}=7.8 \mathrm{~min})$. 
General procedure for preparation of alcohols $\left[{ }^{18} \mathbf{F}\right] 40$ and $\left[{ }^{18} \mathbf{F}\right] 46 .\left[{ }^{18} \mathrm{~F}\right] \mathrm{Fluoride}$ in water was trapped on a Sep-Pak ${ }^{\circledR}$ QMA cartridge, released with $0.5 \mathrm{ml}$ of a solution of Kryptofix 222 $(30 \mathrm{mM})$ and potassium hydrogen carbonate $(30 \mathrm{mM})$ dissolved in acetonitrile: water (85: 15). After removing the solvent by heating at $90{ }^{\circ} \mathrm{C}$ under a stream of nitrogen, acetonitrile $(0.5 \mathrm{ml})$ was added, and the distillation was continued at $90{ }^{\circ} \mathrm{C}$. This procedure was repeated and the reaction vial was subsequently capped. The respective sulfonium triflate precursor dissolved in $\operatorname{DMSO}(0.5 \mathrm{ml})$ was added and the mixture was stirred at $110{ }^{\circ} \mathrm{C}$ for $15 \mathrm{~min}$. The reaction was quenched and further diluted with water to a volume of $20 \mathrm{ml}$. This solution was filtered over a Sep-Pak ${ }^{\circledR}$ SPE C-18 light cartridge. The radiolabeled product was released with $\mathrm{HCl}$ in ethanol $(1.25 \mathrm{M}, 0.5 \mathrm{ml})$. The filtrate was heated at $90{ }^{\circ} \mathrm{C}$ for $15 \mathrm{~min}$. After cooling, the reaction was quenched with water $(1.5 \mathrm{ml})$ and purified by HPLC using a Chromolith ${ }^{\circledR}$ SemiPrep RP18-e column $(100 \times 10 \mathrm{~mm})$ at room temperature. The mobile phase consisted of water and methanol (each containing $0.5 \%$ TFA). Gradient elution starting with $10 \%$ methanol content that was increased to $55 \%$ in $12 \mathrm{~min}$ and further to $90 \%$ in $3 \mathrm{~min}$ allowed for isolation of the radioactive product. The obtained solution was diluted with water to a final volume of $20 \mathrm{ml}$, trapped on a Sep-Pak ${ }^{\circledR}$ SPE C-18 light cartridge, and the radiolabeled product was released with $0.5 \mathrm{ml}$ ethanol. After having reduced the volume to $100 \mu 1$ under a stream of nitrogen, the solution was diluted with saline to give a final ethanol concentration of $5 \%$ and sterilized by filtration.

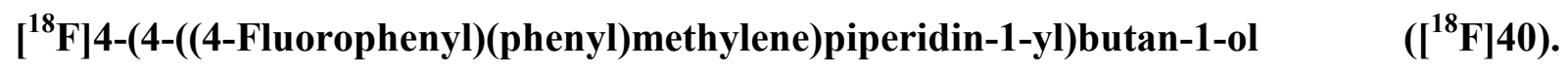
Starting with 1.5-2.5 GBq fluoride-18, labeling of compound $\mathbf{7 0}$ followed by hydrolysis gave $\left[{ }^{18} \mathrm{~F}\right] 40$ in $5-10 \%$ overall RCY. Quality control was performed on a Chromolith ${ }^{\circledR}$ Performance RP18-e column $(100 \times 4.6 \mathrm{~mm})$ using water and methanol (each containing $0.5 \%$ TFA; gradient elution at a flow rate of $3 \mathrm{ml} / \mathrm{min}$ starting with $10 \%$ methanol content that was increased to $55 \%$ 
in $12 \mathrm{~min}$ and further to $90 \%$ in $3 \mathrm{~min}$ ). The radiochemical purity was in the range of $98-100 \%$ and the identity of the radiochemical product was confirmed by co-elution with the nonradioactive analogue $(\mathrm{RT}=12.7 \mathrm{~min})$.

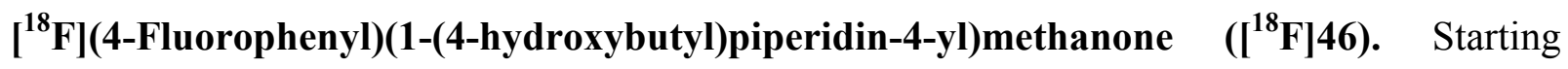
with $1.75 \mathrm{GBq}$ fluoride-18, labeling of compound $\mathbf{6 2}$ followed by hydrolysis gave $36.4 \mathrm{MBq}$ of formulated $\left[{ }^{18} \mathrm{~F}\right] \mathbf{4 6}$ (7\% overall RCY). Quality control was performed by radio-HPLC using an analytical Chromolith ${ }^{\circledR}$ Performance RP18-e column $(100 \times 4.6 \mathrm{~mm})$ using water and methanol (each containing $0.5 \% \mathrm{TFA}$; gradient elution at a flow rate of $3 \mathrm{ml} / \mathrm{min}$ starting with $1 \%$ methanol content that was increased to $40 \%$ in 15 ). The radiochemical purity was $96 \%$ and the identity of the radiochemical product was confirmed by co-elution with the non-radioactive analogue $(\mathrm{RT}=6.8 \mathrm{~min})$.

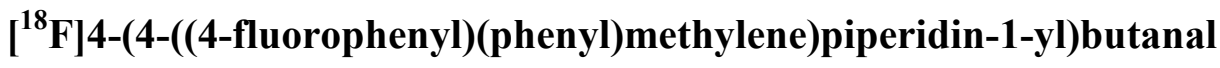

$\left(\left[{ }^{18} \mathrm{~F}\right] 42\right)$.

$\left[{ }^{18} \mathrm{~F}\right]$ Fluoride in water $(1.78 \mathrm{GBq})$ was trapped on a Sep-Pak ${ }^{\circledR}$ QMA cartridge, released with 0.5 $\mathrm{ml}$ of a solution of Kryptofix $222(30 \mathrm{mM})$ and potassium hydrogen carbonate $(30 \mathrm{mM})$ dissolved in acetonitrile: water (85: 15). After removing the solvent by heating at $90{ }^{\circ} \mathrm{C}$ under a stream of nitrogen, acetonitrile $(0.5 \mathrm{ml})$ was added, and the distillation was continued at $90{ }^{\circ} \mathrm{C}$. This procedure was repeated and the reaction vial was subsequently capped. Compound $\mathbf{7 4}$ dissolved in DMSO $(0.5 \mathrm{ml})$ was added and the mixture was stirred at $110{ }^{\circ} \mathrm{C}$ for $15 \mathrm{~min}$. The reaction was quenched and further diluted with water to a volume of $20 \mathrm{ml}$. This solution was filtered over a Sep-Pak ${ }^{\circledR}$ SPE C-18 light cartridge. The radiolabeled product was released with THF $(0.5 \mathrm{ml})$, and $\mathrm{HCl}(1 \mathrm{M} ; 1.5 \mathrm{ml})$ was added. This solution was heated at $90{ }^{\circ} \mathrm{C}$ for $15 \mathrm{~min}$. After cooling, the crude mixture was purified by HPLC using a Chromolith ${ }^{\circledR}$ SemiPrep RP18-e column $(100 \times 10 \mathrm{~mm})$ at room temperature. The mobile phase consisted of water and methanol 
(each containing $0.5 \%$ TFA). Gradient elution starting with $10 \%$ methanol content that was increased to $70 \%$ in $20 \mathrm{~min}$ and further to $90 \%$ in $5 \mathrm{~min}$ allowed for isolation of the radioactive product. The obtained solution was diluted with water to a final volume of $20 \mathrm{ml}$, trapped on a Sep-Pak ${ }^{\circledR}$ SPE C-18 light cartridge, and the radiolabeled product was released with $0.5 \mathrm{ml}$ ethanol. After having reduced the volume to $100 \mu \mathrm{l}$ under a stream of nitrogen, the solution was diluted with saline to give a final ethanol concentration of $5 \%$ and sterilized by filtration resulting in $37.2 \mathrm{MBq}$ of formulated $\left[{ }^{18} \mathrm{~F}\right] 42$ (6\% overall $\left.\mathrm{RCY}\right)$. Quality control was performed by radio-HPLC using an analytical Agilent Zorbax ${ }^{\circledR}$ Eclipse XDB column $(150 \times 4.6 \mathrm{~mm}) \mathrm{using}$ water and methanol (each containing $0.5 \%$ TFA; gradient elution at a flow rate of $3 \mathrm{ml} / \mathrm{min}$ starting with $30 \%$ methanol content that was increased to $90 \%$ in 15 ). The radiochemical purity was $96 \%$ and the identity of the radiochemical product was confirmed by co-elution with the non-radioactive analogue $(\mathrm{RT}=16.4 \mathrm{~min})$.

General procedure for preparation of esters $\left[{ }^{18} \mathbf{F}\right] 43$ and $\left[{ }^{18} \mathbf{F}\right] 47 .\left[{ }^{18} \mathrm{~F}\right]$ Fluoride in water was trapped on a Sep-Pak ${ }^{\circledR}$ QMA cartridge, released with $0.5 \mathrm{ml}$ of a solution of Kryptofix 222 (30 $\mathrm{mM})$ and potassium hydrogen carbonate $(30 \mathrm{mM})$ dissolved in acetonitrile: water (85: 15). After removing the solvent by heating at $90{ }^{\circ} \mathrm{C}$ under a stream of nitrogen, acetonitrile $(0.5 \mathrm{ml})$ was added, and the distillation was continued at $90{ }^{\circ} \mathrm{C}$. This procedure was repeated and the reaction vial was subsequently capped. The respective sulfonium triflate dissolved in DMSO (0.5 ml) was subsequently added and the mixture was stirred at $110{ }^{\circ} \mathrm{C}$ for $15 \mathrm{~min}$. The reaction was quenched with water $(1.5 \mathrm{ml})$ and the mixture was purified by HPLC using a Chromolith ${ }^{\circledR}$ SemiPrep RP18e column $(100 \times 10 \mathrm{~mm})$ at room temperature. The mobile phase consisted of water and methanol (each containing 0.5\% TFA). Gradient elution starting with 10\% methanol content that was increased to $55 \%$ in $12 \mathrm{~min}$ and further to $90 \%$ in $3 \mathrm{~min}$ allowed for isolation of the 
radioactive product. The obtained solution was diluted with water to a final volume of $20 \mathrm{ml}$, trapped on a Sep-Pak ${ }^{\circledR}$ SPE C-18 light cartridge, and the radiolabeled product was released with $0.5 \mathrm{ml}$ ethanol. After having reduced the volume to $100 \mu \mathrm{l}$ under a stream of nitrogen, the solution was diluted with saline to give a final ethanol concentration of $5 \%$ and sterilized by filtration.

\section{$\left[{ }^{18}\right.$ F]Ethyl 4-(4-((4-fluorophenyl)(phenyl)methylene)piperidin-1-yl)butanoate $\quad\left(\left[{ }^{18} \mathrm{~F}\right] 43\right)$.} Starting with $1.7 \mathrm{GBq}$ fluoride-18, labeling of compound 72 gave $26-30 \mathrm{MBq}(\mathrm{n}=2)$ of formulated $\left[{ }^{18} \mathrm{~F}\right] 43$ (3\% overall RCY). Quality control was performed on a Chromolith ${ }^{\circledR}$ Performance RP18-e column $(100 \times 4.6 \mathrm{~mm})$ using water and methanol (each containing 0.5\% TFA; gradient elution at a flow rate of $3 \mathrm{ml} / \mathrm{min}$ starting with $10 \%$ methanol content that was increased to $55 \%$ in $12 \mathrm{~min}$ and further to $90 \%$ in $3 \mathrm{~min}$ ). The radiochemical purity was $99 \%$ and the identity of the radiochemical product was confirmed by co-elution with the non-radioactive analogue $(\mathrm{RT}=11.6 \mathrm{~min})$.

$\left[{ }^{18}\right.$ F $]$ Ethyl 4-(4-(4-fluorobenzoyl)piperidin-1-yl)butanoate $\left(\left[{ }^{18} \mathbf{F}\right] 47\right)$. Starting with $1.4 \mathrm{GBq}$ fluoride-18, labeling of compound 64 gave $136 \mathrm{MBq}$ of formulated $\left[{ }^{18} \mathrm{~F}\right] 47$ (18\% overall RCY). Quality control was performed on a Chromolith ${ }^{\circledR}$ Performance RP18-e column $(100 \times 4.6 \mathrm{~mm})$ using water and methanol (each containing $0.5 \%$ TFA; gradient elution at a flow rate of $3 \mathrm{ml} / \mathrm{min}$ starting with $10 \%$ methanol content that was increased to $55 \%$ in 12 min and further to $90 \%$ in 3 min). The radiochemical purity was $100 \%$ and the identity of the radiochemical product was confirmed by co-elution with the non-radioactive analogue $(\mathrm{RT}=10.2 \mathrm{~min})$.

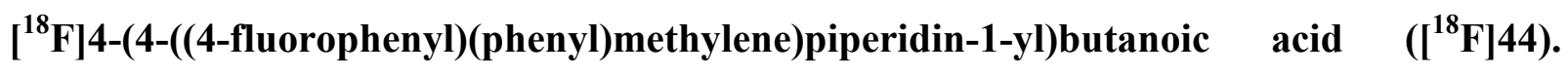
$\left[{ }^{18} \mathrm{~F}\right] 44$ was prepared from $\left[{ }^{18} \mathrm{~F}\right] 43$ using the synthetic procedure described above. After the labeling reaction, the crude mixture was diluted with water to a volume of $20 \mathrm{ml}$ and filtered
} 
over a Sep-Pak ${ }^{\circledR}$ SPE C-18 light cartridge. The radiolabeled product was released from the cartridge with ethanol $(1.0 \mathrm{ml})$, to which was added aqueous $\mathrm{NaOH}(1 \mathrm{M} ; 0.5 \mathrm{ml})$. The ester was hydrolysed in an open vial for $15 \mathrm{~min}$ at $90{ }^{\circ} \mathrm{C}$ and the resulting carboxylic acid was subsequently purified by HPLC using a Chromolith ${ }^{\circledR}$ SemiPrep RP18-e column $(100 \times 10 \mathrm{~mm})$ at room temperature. The mobile phase consisted of water and methanol (each containing $0.5 \%$ TFA). Gradient elution starting with $10 \%$ methanol content that was increased to $55 \%$ in $12 \mathrm{~min}$ and further to $90 \%$ in $3 \mathrm{~min}$ allowed for isolation of the radioactive product. The isolated product was re-formulated as described above. Starting with 1.0-1.6 GBq fluoride-18, the two-step synthetic procedure afforded $10-15 \mathrm{MBq}$ of formulated $\left[{ }^{18} \mathrm{~F}\right] 44(2.3-2.6 \%$ overall $\mathrm{RCY})$. Quality control was performed on a Chromolith ${ }^{\circledR}$ Performance RP18-e column $(100 \times 4.6 \mathrm{~mm})$ using water and methanol (each containing $0.5 \%$ TFA; gradient elution at a flow rate of $3 \mathrm{ml} / \mathrm{min}$ starting with $10 \%$ methanol content that was increased to $55 \%$ in 12 min and further to $90 \%$ in 3 min). The radiochemical purity was $100 \%$ and the identity of the radiochemical product was confirmed by co-elution with the non-radioactive analogue $(\mathrm{RT}=8.7 \mathrm{~min})$.

P-gp ATPase activity assay. The Pgp-Glo ${ }^{\mathrm{TM}}$ assay (Promega) was performed following the manufacturer's instructions. ${ }^{34}$ Briefly, a membrane preparation from Sf9 cells expressing the recombinant human P-gp was incubated with the respective test compound (20 $\mu \mathrm{M})$ and ATP $(5$ $\mathrm{mM})$ for 40 minutes at $37^{\circ} \mathrm{C}$. Sodium orthovanadate $(20 \mu \mathrm{M})$ and verapamil $(200 \mu \mathrm{M})$ were added as negative and positive control, respectively. After this time, the enzyme luciferase and the corresponding substrate luciferin were added, and incubation was continued for 20 minutes. Quantification was carried out by determination of luminescence. Results are given as reduction of luminescence (the more avid a substrate the more pronounced is the reduction of luminescence). 
$\boldsymbol{h} \mathbf{H}_{1} \mathbf{R}$ binding assay. $\mathrm{CHO}-\mathrm{K} 1$ cells stably expressing the $h \mathrm{H}_{1} \mathrm{R}$ were washed with $10 \mathrm{ml}$ icecold PBS buffer (140 mM NaCl, 3 mM KCl, $1.5 \mathrm{mM} \mathrm{KH}_{2} \mathrm{PO}_{4}, 8 \mathrm{mM} \mathrm{NaHPO}$, pH 7.4), scraped into ice-cold HEPES binding buffer (20 mM HEPES, $10 \mathrm{mM} \mathrm{MgCl}_{2}, 100 \mathrm{mM} \mathrm{NaCl}, \mathrm{pH}$ 7.4), and homogenized with sonication. Membranes were pelleted at 23,000 x g for 30 minutes at 4 ${ }^{\circ} \mathrm{C}$, homogenized in HEPES buffer using a hand potter, and stored in liquid nitrogen. Prior to experiments, cell membranes were thawed, homogenized with sonication at $4{ }^{\circ} \mathrm{C}$ into ice-cold HEPES binding buffer. Competition binding experiments were carried out incubating membranes, $35 \mu \mathrm{g} /$ well in a final volume of $0.2 \mathrm{ml}$ containing binding buffer and $\left[{ }^{3} \mathrm{H}\right]$ pyrilamine (1.0 $\mathrm{nM} ; 27 \mathrm{Ci} / \mathrm{mmol})$. Assays were run in triplicates with at least four appropriate concentrations between $1 \mathrm{nM}$ and $1 \mu \mathrm{M}$ of the test compound. Incubations were performed for $120 \mathrm{~min}$ at $25^{\circ} \mathrm{C}$ and shaking at $250 \mathrm{rpm}$. Non-specific binding was determined in the presence of $10 \mu \mathrm{M}$ chlorpheniramine hydrogenmaleate. Bound radioligand was separated from free radioligand by filtration through $\mathrm{GF} / \mathrm{B}$ filters pre-treated with $0.3 \%$ (mass/vol) polyethyleneimine using an Inotech cell harvester. Unbound radioligand was removed with four washes of $5 \mathrm{ml}$ of ice-cold HEPES buffer. ${ }^{35}$

Animals. All animal work at UCL was performed in compliance with the United Kingdom Home Office's Animals (Scientific Procedures) Act 1986 and with approval of the University College London (UCL) Animal Ethics Committee. Female wild-type albino mice (FVB or Balb/C, Charles River Laboratories, Margate, UK) were allowed to acclimatize for at least one week at the animal facilities at the UCL Centre for Advanced Biomedical Imaging, and they were given food and water ad libitum. When used for experiments, they were eight to eleven weeks old and weighing approximately $20 \mathrm{~g}$. For PET scanning experiments at AIT, wild-type FVB as well as P-gp knock-out mice were obtained from Taconic (Germantown, USA). The 
study was approved by the local animal welfare committee (Amt der Niederösterreichischen Landesregierung) and all experimental procedures were performed in accordance with the European Communities Council Directive of September 22, 2010 (2010/63/EU).

Biodistribution Studies. Biodistribution studies were performed at UCL's Centre for Advanced Biomedical Imaging. The respective radiotracer $(0.5-3 \mathrm{MBq}$ formulated in saline solution containing max. $5 \%$ ethanol) was administered intravenously into the tail vein of female wild type albino mice (Balb/C or FVB) without anesthesia. At designated time points between five minutes and two hours after tracer injection, animals were anesthetized with isoflurane (5\% mixed with medical air at a flow of $2 \mathrm{ml} / \mathrm{min}$ ) and the blood was taken by cardiac puncture. Mice were subsequently sacrificed by cervical dislocation. The organs of interest were sampled, weighed, and the radioactivity content was measured by automated gamma counting (Perkin Elmer Wizard ${ }^{2}$ ). Results were expressed as \% ID/g bodyweight. All experiments were performed in duplicate or triplicate. For blocking studies with tariquidar, $15 \mathrm{mg} / \mathrm{kg}$ of the P-gp inhibitor formulated in $300 \mu \mathrm{l}$ of a $2.5 \%$ glucose solution was administered intravenously into the tail vein two hours before tracer injection. The dose was administered slowly over the course of approximately $2 \mathrm{~min}$.

Metabolite Analysis. Metabolite analysis was performed as part of the biodistribution studies. Blood samples were collected in heparin-coated tubes and an aliquot $(20 \mu \mathrm{l})$ was taken for gamma counting. After centrifugation ( $3 \mathrm{~min}, 13,000 \mathrm{rpm}$ ), the plasma was separated. Plasma proteins were subsequently precipitated with cold ethanol (500 $\mu 1)$ and samples were centrifuged ( $3 \mathrm{~min}, 13,000 \mathrm{rpm}$ ). $500 \mu \mathrm{l}$ of the resulting supernatant were separated from the pellet, diluted with water $(500 \mu \mathrm{l})$ and analyzed by radio-HPLC, using a Phenomenex Luna ${ }^{\circledR}$ C-8 (5 $\mu \mathrm{m}, 150 \mathrm{x}$ $4.6 \mathrm{~mm})$ or an Agilent Zorbax ${ }^{\circledR}$ Eclipse XDB C-18 $(5 \mu \mathrm{m}, 100 \times 4.6 \mathrm{~mm})$ analytical column at 
room temperature. The mobile phase consisted of water and methanol containing $0.02 \% \mathrm{NH}_{4} \mathrm{OH}$ and was used for gradient elution (from 30\% of methanol to $95 \%$ in $15 \mathrm{~min}$, followed by isocratic elution with $95 \%$ for $5 \mathrm{~min}$ ). The flow rate was $1 \mathrm{ml} / \mathrm{min}$ and the $\mathrm{UV}$ absorbance detector was set at $254 \mathrm{~nm}$. Brains were homogenized in a mixture of water (500 $\mu 1)$ and ethanol $(500 \mu 1)$. Samples were centrifuged $(3 \mathrm{~min}, 13,000 \mathrm{rpm})$, the resulting supernatant was separated and deproteinated by adding ethanol a second time $(500 \mu 1)$. After vortexing and centrifugation (3 min, 13,000 rpm), the clear supernatant (500 $\mu \mathrm{l})$ was separated from the pellet, diluted with water $(500 \mu \mathrm{l})$ and analyzed by radio-HPLC, using the method reported above. The recovery of radioactivity in the investigated samples from both plasma and brain was almost quantitative (> 95\%). All experiments were performed in triplicate.

PET scans. Dynamic PET imaging was performed using a $\mu$ PET Focus220 scanner (Siemens Medical Solutions, Knoxville, TN) at AIT and a nanoScan ${ }^{\circledR}$ PET-CT system manufactured by Mediso (Medical Imaging Systems, Budapest, Hungary) at UCL, respectively. Mice were anaesthetized with isoflurane (2\% in oxygen) and placed on the preheated bed of the scanner (set at $\left.38^{\circ} \mathrm{C}\right)$. The respective radiotracer $(5-10 \mathrm{MBq}$ in $100-250 \mu \mathrm{l}$ saline solution) was injected into the tail vein via intravenous cannulation. After injection, the catheter was carefully removed. Breathing rate and body temperature of the animals were closely monitored during the dynamic PET scans and, if necessary, the isoflurane dose was adjusted. Scans were recorded over two hours, and the animals were subsequently sacrificed by cervical dislocation. Quantification of tissue uptake was carried out using the image analysis software Amide at AIT and the software package VivoQuant 1.23 (inviCRO, Boston, USA) at UCL, respectively.

Blocking studies. Tariquidar $(15 \mathrm{mg} / \mathrm{kg}$ bodyweight; formulated in $2.5 \%$ glucose solution $)$ or elacridar (10 mg/kg bodyweight; formulated in $20 \%$ ethanol in water) were injected slowly (over 
the course of 1-2 min) into the tail vein 90 min before tracer administration in order to achieve full inhibition of the respective efflux transporters. Control animals received vehicle or saline. This was carried out without anesthesia, and mice were allowed food and water ad libitum in between the injections.

Analysis of tracer kinetics. In the absence of an arterial input function, we used a simple model to describe the uptake of the pro-drug tracer from the blood, metabolism and trapping of the tracer in the brain, and the washout of the metabolically activated tracer. Whole-brain timeactivity curves were analyzed by fitting the sum of a mono-exponential function and a constant to the time-activity curve data from $10 \mathrm{~min}$ p.i. The amplitude of the exponential term $A_{1}$ would correspond to tracer being washed out from the brain with $s$ representing the efflux rate, and the constant term $A_{2}$ to tracer that gets trapped in brain tissue.

$$
C(t)=A_{1} e^{-s t}+A_{2}, \quad t>10 \min
$$

\section{AUTHOR INFORMATION}

Corresponding Author. * Phone: +44 207 6792344; Email: e.arstad@ucl.ac.uk

Present Author Address. \# Heinrich Heine University Duesseldorf, Institute of Pharmaceutical and Medicinal Chemistry, Universitaetsstrasse 1, 40225 Duesseldorf, Germany

\section{ACKNOWLEDGMENT}

The research leading to these results has received funding from the European Community's Seventh Framework Programme (FP7/2007-2013) under grant agreement number 201380 (“Euripides"). 


\begin{abstract}
ABBREVIATIONS
$\mathrm{ABC}$, adenosine triphosphate-binding cassette; $\mathrm{BBB}$, blood-brain barrier; BCRP, breast cancer resistance protein; CNS, central nervous system; $h \mathrm{H}_{1} \mathrm{R}$, human histamine $\mathrm{H}_{1}$ receptor; HPLC, high performance liquid chromatography; ID/g, injected dose per gram tissue; MDR, multiple drug resistance; MEM, metabolite extrusion method; PET, positron emission tomography; P-gp, P-glycoprotein; p.i., post injection; RCY, radiochemical yield.
\end{abstract}




\section{REFERENCES}

1. Miller, D. S., Regulation of ABC transporters at the blood-brain barrier. Clin. Pharmacol. Ther. 2015, 97, 395-403.

2. (a) Seelig, A., A general pattern for substrate recognition by P-glycoprotein. Eur. J. Biochem. 1998, 251, 252-261; (b) Penzotti, J. E.; Lamb, M. L.; Evensen, E.; Grootenhuis, P. D. J., A computational ensemble pharmacophore model for identifying substrates of Pglycoprotein. L.Med.Chem. 2002, 45, 1737-1740.

3. (a) International Transporter Consortium, Membrane transporters in drug development. Nat.Rev.Drug Discoverv 2010, 9, 215-236; (b) Robey, R. W.; Massey, P. R.; AmiriKordestani, L.; Bates, S. E., ABC transporters: unvalidated therapeutic targets in cancer and the CNS. Anticancer Agents Med.Chem. 2010, 10, 625-633.

4. (a) Agarwal, S.; Elmquist, W. F., Insight into the cooperation of P-glycoprotein (ABCB1) and breast cancer resistance protein $(\mathrm{ABCG})$ at the blood-brain barrier: a case study examining sorafenib efflux clearance. Mol. Pharmaceutics 2012, 9, 678-684; (b) de Vries, N. A.; Zhao, J.; Kroon, E.; Buckle, T.; Beijnen, J. H.; van Tellingen, O., Pglycoprotein and breast cancer resistance protein: two dominant transporters working together in limiting the brain penetration of topotecan. Clin. Cancer Res. 2007, 13, 64406449; (c) Mittapalli, R. K.; Vaidhyanathan, S.; Sane, R.; Elmquist, W. F., Impact of Pglycoprotein $(\mathrm{ABCB} 1)$ and breast cancer resistance protein $(\mathrm{ABCG} 2)$ on the brain distribution of a novel BRAF inhibitor: vemurafenib (PLX4032). J. Pharmacol. Exp. Ther. 2012, 342, 33-40.

5. (a) Kubota, H.; Ishihara, H.; Langmann, T.; Schmitz, G.; Stieger, B.; Wieser, H.-G.; Yonekawa, Y.; Frei, K., Distribution and functional activity of P-glycoprotein and 
multidrug resistance-associated proteins in human brain microvascular endothelial cells in hippocampal sclerosis. Enilensv Res. 2006, 68, 213-228; (b) Feldmann, M.; Asselin, M.-C.; Liu, J.; Wang, S.; McMahon, A.; Anton-Rodriguez, J.; Walker, M.; Symms, M.; Brown, G.; Hinz, R.; Matthews, J.; Bauer, M.; Langer, O.; Thom, M.; Jones, T.; Vollmar, C.; Duncan, J. S.; Sisodiya, S. M.; Koepp, M. J., P-glycoprotein expression and function in patients with temporal lobe epilepsy: a case-control study. Lancet Neurol. 2013, 12, 777-785; (c) Xiong, H.; Callaghan, D.; Jones, A.; Bai, J.; Rasquinha, I.; Smith, C.; Pei, K.; Walker, D.; Lue, L. F.; Stanimirovic, D.; Zhang, W., ABCG2 is upregulated in Alzheimer's brain with cerebral amyloid angiopathy and may act as a gatekeeper at the blood-brain barrier for Abeta(1-40) peptides. J. Neurosci. 2009, 29, 5463-5475; (d) Krishnamurthy, P.; Ross, D. D.; Nakanishi, T.; Bailey-Dell, K.; Zhou, S.; Mercer, K. E.; Sarkadi, B.; Sorrentino, B. P.; Schuetz, J. D., The stem cell marker Bcrp/ABCG2 enhances hypoxic cell survival through interactions with heme. J. Biol. Chem. 2004, 279, 24218-24225; (e) Natarajan, K.; Xie, Y.; Baer, M. R.; Ross, D. D., Role of breast cancer resistance protein (BCRP/ABCG2) in cancer drug resistance. Biochem. Pharmacol. 2012, $83,1084-1103$.

6. (a) Hermann, D. M.; Kilic, E.; Spudich, A.; Kramer, S. D.; Wunderli-Allenspach, H.; Bassetti, C. L., Role of drug efflux carriers in the healthy and diseased brain. Ann. Neurol. 2006, 60, 489-498; (b) Löscher, W.; Potschka, H., Drug resistance in brain diseases and the role of drug efflux transporters. Nat.Rev. Neurosci. 2005, 6, 591-602; (c) Urquhart, B. L.; Kim, R. B., Blood-brain barrier transporters and response to CNSactive drugs. Eur. J. Clin. Pharmacol. 2009, 65, 1063-1070. 
7. Löscher, W.; Potschka, H., Role of drug efflux transporters in the brain for drug disposition and treatment of brain diseases. Prog. Neurobiol. 2005, 76, 22-76.

8. (a) Müllauer, J.; Karch, R; Bankstahl, J. P.; Bankstahl, M.; Stanek, J.; Wanek, T.; Mairinger, S.; Müller, M.; Löscher, W.; Langer, O.; Kuntner, C., Assessment of cerebral P-glycoprotein expression and function with PET by combined $\left[{ }^{11} \mathrm{C}\right]$ inhibitor and $\left[{ }^{11} \mathrm{C}\right]$ substrate scans in rats. Nucl.Med.Biol. 2013, 40, 755-763; (b) Kannan, P.; Telu, S.; Shukla, S.; Ambudkar, S.V.; Pike, V.W.; Halldin, C.; Gottesman, M.M.; Innis, R.B.; Hall, M.D., The "specific" P-glycoprotein inhibitor tariquidar is also a substrate and an inhibitor for breast cancer resistance protein (BCRP/ABCG2). ACS Chem. Neurosci. 2011, 2, 82-89; (c) Bankstahl, J. P.; Bankstahl, M.; Römermann, K.; Wanek, T.; Stanek, J.; Windhorst, A. D.; Fedrowitz, M.; Erker, T.; Müller, M.; Löscher, W.; Langer, O.; Kuntner, C., Tariquidar and elacridar are dose-dependently transported by P-glycoprotein and Bcrp at the blood-brain barrier: a small-animal positron emission tomography and in vitro study. Drug Metab.Dispos. 2013, 41, 754-462.

9. (a) Colabufo, N. A.; Berardi, F.; Cantore, M.; Contino, M.; Inglese, C.; Niso, M.; Perrone, R., Perspectives of P-glycoprotein modulating agents in oncology and neurodegenerative diseases: pharmaceutical, biological, and diagnostic potentials. J. Med. Chem. 2010, 53, 1883-1897; (b) Kannan, P.; John, C.; Zoghbi, S. S.; Halldin, C.; Gottesman, M. M.; Innis, R. B.; Hall, M. D., Imaging the function of P-glycoprotein with radiotracers: pharmacokinetics and in vivo applications. Clin. Pharmacol. Ther. 2009, 86, 368-377; (c) Mairinger, S.; Erker, T.; Muller, M.; Langer, O., PET and SPECT radiotracers to assess function and expression of $\mathrm{ABC}$ transporters in vivo. Curr. Drug Metab. 2011, 12, 774-792. 
10. (a) Okamura, T.; Kikuchi, T.; Okada, M.; Toramatsu, C.; Fukushi, K.; Takei, M.; Irie, T., Noninvasive and quantitative assessment of the function of multidrug resistanceassociated protein 1 in the living brain. L_Cereb. Blood Flow Metab. 2009, 29, 504-511; (b) Galante, E.; Okamura, T.; Sander, K.; Kikuchi, T.; Okada, M.; Zhang, M. R.; Robson, M.; Badar, A.; Lythgoe, M.; Koepp, M.; Arstad, E., Development of purine-derived ${ }^{18}$ Flabeled pro-drug tracers for imaging of MRP1 activity with PET. J. Med. Chem. 2014, 57, 1023-1032.

11. Pavan, B.; Dalpiaz, A.; Ciliberti, N.; Biondi, C.; Manfredini, S.; Vertuani, S., Progress in drug delivery to the central nervous system by the prodrug approach. Molecules 2008, 13, $1035-65$.

12. (a) Zhao, R.; Kalvass, J. C.; Yanni, S. B.; Bridges, A. S.; Pollack, G. M., Fexofenadine brain exposure and the influence of blood-brain barrier P-glycoprotein after fexofenadine and terfenadine administration. Drug Metab. Dispos. 2009, 37, 529-535; (b) Chen, C.; Hanson, E.; Watson, J. W.; Lee, J. S., P-glycoprotein limits the brain penetration of nonsedating but not sedating $\mathrm{H}_{1}$-antagonists. Drug Metab.Dispos. 2003, 31, 312-318; (c) Mahar Doan, K. M.; Wring, S. A.; Shampine, L. J.; Jordan, K. H.; Bishop, J. P.; Kratz, J.; Yang, E.; Serabjit-Singh, C. J.; Adkison, K. K.; Polli, J. W., Steady-state brain concentrations of antihistamines in rats: interplay of membrane permeability, Pglycoprotein efflux and plasma protein binding. Pharmacologv 2004, 72, 92-98; (d) Obradovic, T.; Dobson, G. G.; Shingaki, T.; Kungu, T.; Hidalgo, I. J., Assessment of the first and second generation antihistamines brain penetration and role of P-glycoprotein. Pharm. Res. 2007, 24, 318-327. 
13. Renwick, A. G., The metabolism of antihistamines and drug interactions: the role of cytochrome P450 enzymes. Clin. Exp. Allergy 1999, 29 Suppl 3, 116-124.

14. Sander, K.; Kottke, T.; Hoffend, C.; Walter, M.; Weizel, L.; Camelin, J. C.; Ligneau, X.; Schneider, E. H.; Seifert, R.; Schwartz, J. C.; Stark, H., First metal-containing histamine $\mathrm{H}_{3}$ receptor ligands. Org Lett. 2010, 12, 2578-2581.

15. Omura, K.; Swern, D., Oxidation of alcohols by "activated" dimethyl sulfoxide. a preparative, steric and mechanistic study. Tetrahedron 1978, 34, 1651-1660.

16. Shiuey, S. J.; Partridge, J. J.; Uskokovic, M. R., Triply convergent synthesis of 1 $\alpha, 25-$ dihydroxy-24(R)-fluorocholecalciferol. L. Org.Chem. 1988, 53, 1040-1046.

17. (a) Ohta, A.; Sawamoto, D.; Jayasundera, K. P.; Kinoshita, H.; Inomata, K., Efficient synthesis of B- and C-rings components of phycobilin derivatives for structure/function analysis of phytochrome. Chem. Lett. 2000, 29, 492-493; (b) Tamura, S.; Kaneko, M.; Shiomi, A.; Yang, G. M.; Yamaura, T.; Murakami, N., Unprecedented NES nonantagonistic inhibitor for nuclear export of Rev from Sida cordifolia. Bioorg. Med. Chem. Lett. 2010, 20, 1837-1839.

18. Riss, P. J.; Hummerich, R.; Schloss, P., Synthesis and monoamine uptake inhibition of conformationally constrained $2 \beta$-carbomethoxy-3 $\beta$-phenyl tropanes. Org. Biomol. Chem. 2009, 7, 2688-2698.

19. (a) Iradier, F.; Arrayás, R. G.; Carretero, J. C., Synthesis of medium-sized cyclic amines by selective ring cleavage of sulfonylated bicyclic amines. Org Lett. 2001, 3, 2957-2960; (b) Zhou, J.; Zhang, A.; Klass, T.; Johnson, K. M.; Wang, C. Z.; Ye, Y. P.; Kozikowski, A. P., Biaryl analogues of conformationally constrained tricyclic tropanes as potent and 
selective norepinephrine reuptake inhibitors: synthesis and evaluation of their uptake inhibition at monoamine transporter sites. L.Med.Chem. 2003, 46, 1997-2007.

20. Proschak, E.; Sander, K.; Zettl, H.; Tanrikulu, Y.; Rau, O.; Schneider, P.; SchubertZsilavecz, M.; Stark, H.; Schneider, G., From molecular shape to potent bioactive agents II: fragment-based de novo design. ChemMedChem 2009, 4, 45-48.

21. Laine, D. I.; McCleland, B.; Thomas, S.; Neipp, C.; Underwood, B.; Dufour, J.; Widdowson, K. L.; Palovich, M. R.; Blaney, F. E.; Foley, J. J.; Webb, E. F.; Luttmann, M. A.; Burman, M.; Belmonte, K.; Salmon, M., Discovery of novel 1azoniabicyclo[2.2.2]octane muscarinic acetylcholine receptor antagonists. J. Med. Chem. 2009, 52, 2493-2505.

22. Uto, Y.; Ogata, T.; Kiyotsuka, Y.; Ueno, Y.; Miyazawa, Y.; Kurata, H.; Deguchi, T.; Watanabe, N.; Konishi, M.; Okuyama, R.; Kurikawa, N.; Takagi, T.; Wakimoto, S.; Ohsumi, J., Novel benzoylpiperidine-based stearoyl-CoA desaturase-1 inhibitors: Identification of 6-[4-(2-methylbenzoyl)piperidin-1-yl]pyridazine-3-carboxylic acid (2hydroxy-2-pyridin-3-ylethyl)amide and its plasma triglyceride-lowering effects in Zucker fatty rats. Bioorg. Med. Chem. Lett. 2010, 20, 341-345.

23. (a) Tredwell, M.; Gouverneur, V., ${ }^{18}$ F Labeling of arenes. Angew. Chem. Int. Ed. 2012, 51, 11426-11437; (b) Brooks, A. F.; Topczewski, J. J.; Ichiishi, N.; Sanford, M. S.; Scott, P. J., Late-stage $\left[{ }^{18}\right.$ F]fluorination: new solutions to old problems. Chem. Sci. 2014, 5, 4545-4553.

24. Mu, L.; Fischer, C. R.; Holland, J. P.; Becaud, J.; Schubiger, P. A.; Schibli, R.; Ametamey, S. M.; Graham, K., Stellfeld, T.; Dinkelborg, L. M.; Lehmann, L., ${ }^{18}$ F- 
Radiolabeling of aromatic compounds using triarylsulfonium salts. Eur. J. Org. Chem. 2012, 2012, 889-892.

25. Sander, K.; Gendron, T.; Yiannaki, E.; Cybulska, K.; Kalber, T. L.; Lythgoe, M. F.; Arstad, E., Sulfonium salts as leaving groups for aromatic labelling of drug-like small molecules with fluorine-18. Sci.Rep. 2015, 5, 9941.

26. (a) Zhu, M.; Jalalian, N.; Olofsson, B., One-pot synthesis of diaryliodonium salts using toluenesulfonic acid: a fast entry to electron-rich diaryliodonium tosylates and triflates. Svnlett 2008, 4, 592-596; (b) Kazmierczak, P.; Skulski, L., Oxidative anion metatheses in diaryliodonium iodides and chlorides. Bull.Chem.Soc.Jpn. 1997, 70, 219-224.

27. Schwab, D.; Fischer, H.; Tabatabaei, A.; Poli, S.; Huwyler, J., Comparison of in vitro Pglycoprotein screening assays: recommendations for their use in drug discovery. J. Med. Chem. 2003, 46, 1716-1725.

28. Kuntner, C.; Bankstahl, J. P.; Bankstahl, M.; Stanek, J.; Wanek, T.; Stundner, G.; Karch, R.; Brauner, R.; Meier, M.; Ding, X.; Müller, M.; Löscher, W.; Langer, O., Doseresponse assessment of tariquidar and elacridar and regional quantification of Pglycoprotein inhibition at the rat blood-brain barrier using $(R)-\left[{ }^{11} \mathrm{C}\right]$ verapamil PET. Eur. L.Nucl.Med.Mol.Jmaging 2010, 37, 942-953.

29. Gunn, R. N.; Gunn, S. R.; Cunningham, V. J., Positron emission tomography compartmental models. L.Cereb. Blood Flow Metab. 2001, 21, 635-652.

30. Matsson, P.; Pedersen, J. M.; Norinder, U.; Bergström, C. A. S.; Artursson, P., Identification of novel specific and general inhibitors of the three major human ATPbinding cassette transporters P-gp, BCRP and MRP2 among registered drugs. Pharm. Res. 2009, 26, 1816-1831. 
31. (a) Martignoni, M.; Groothuis, G. M.; de Kanter, R., Species differences between mouse, rat, dog, monkey and human CYP-mediated drug metabolism, inhibition and induction. Expert Opin. Drug Metab. Toxicol. 2006, 2, 875-894; (b) Warren, M. S.; Zerangue, N.; Woodford, K.; Roberts, L. M.; Tate, E. H.; Feng, B.; Li, C.; Feuerstein, T. J.; Gibbs, J.; Smith, B.; de Morais, S. M.; Dower, W. J.; Koller, K. J., Comparative gene expression profiles of $\mathrm{ABC}$ transporters in brain microvessel endothelial cells and brain in five species including human. Pharmacol.Res. 2009, 59, 404-413; (c) Syvänen, S.; Lindhe, Ö.; Palner, M.; Kornum, B. R.; Rahman, O.; Långström, B.; Knudsen, G. M.; Hammarlund-Udenaes, M., Species differences in blood-brain barrier transport of three positron emission tomography radioligands with emphasis on P-glycoprotein transport. Drug Metab. Dispos. 2009, 37, 635-643.

32. Walsh, D. A.; Franzyshen, S. K.; Yannil, J. M., Synthesis and antiallergy activity of 4(Diarylhydroxymethyl)-1-[3-(aryloxy)propyl]piperidines and structurally related compounds. J.Med.Chem. 1989, 32, 105-118.

33. Takahashi, S.; Hongo, Y.; Tsukagoshi, Y.; Koshino, H., Structural determination of Montanacin D by total synthesis. Org. Lett. 2008, 10, 4223-4226.

34. Promega, Pgp-Glo ${ }^{\mathrm{TM}}$ Assay Systems. Technical Bulletin 2009; available through https://www.promega.com/ /media/files/resources/protocols/technical\%20bulletins/101/p gp\%20glo\%20assay\%20systems\%20protocol.pdf (June 2015).

35. Smit, M. J.; Timmerman, H.; Hijzelendoorn, J. C.; Fukui, H.; Leurs, R., Regulation of the human histamine $\mathrm{H}_{1}$ receptor stably expressed in Chinese hamster ovary cells. $\mathrm{Br} . J$. Pharmacol. 1996, 117, 1071-1080. 
TABLE

Table 1. Blood and Brain Uptake of Tracer Candidates ${ }^{\text {a) }}$

\begin{tabular}{|c|c|c|c|c|c|c|c|c|}
\hline \multirow[t]{4}{*}{ Compound } & \multirow[t]{4}{*}{ Scaffold } & \multirow[t]{4}{*}{ Pro-drug moiety } & \multicolumn{4}{|c|}{ Tissue uptake (\% ID/g) } & \multicolumn{2}{|c|}{ Brain/blood } \\
\hline & & & \multicolumn{2}{|c|}{ Blood } & \multicolumn{2}{|c|}{ Brain } & & \\
\hline & & & 5 & 30 & 5 & 30 & 5 & 30 \\
\hline & & & \multicolumn{2}{|c|}{$\min$ p.i. } & \multicolumn{2}{|c|}{$\min$ p.i. } & \multicolumn{2}{|c|}{$\min$ p.i. } \\
\hline$\left[{ }^{18} \mathrm{~F}\right] 23$ & & & 1.8 & 1.4 & 5.8 & 1.6 & 3.2 & 1.1 \\
\hline$\left[{ }^{18} \mathrm{~F}\right] 27$ & & & 2.3 & 3.5 & 7.2 & 4.2 & 3.1 & 1.2 \\
\hline$\left[{ }^{18} \mathrm{~F}\right] 38$ & & & 0.8 & 0.8 & 1.8 & 1.8 & 2.3 & 2.3 \\
\hline$\left[{ }^{18} \mathrm{~F}\right] 39$ & & & 0.8 & 0.7 & 1.0 & 1.0 & 1.3 & 1.4 \\
\hline$\left[{ }^{18} \mathrm{~F}\right] 40$ & & & 0.9 & 1.0 & 9.4 & 7.3 & 10.4 & 7.3 \\
\hline$\left[{ }^{18} \mathrm{~F}\right] 42$ & & & 1.4 & 1.6 & 3.1 & 3.4 & 2.2 & 2.1 \\
\hline$\left[{ }^{18} \mathrm{~F}\right] 43$ & & & 2.5 & 3.7 & 2.9 & 4.9 & 1.2 & 1.3 \\
\hline$\left[{ }^{18} \mathrm{~F}\right] 44$ & & $\pi_{0}^{O H}$ & 3.0 & 1.5 & 1.3 & 1.7 & 0.4 & 1.1 \\
\hline$\left[{ }^{18} \mathrm{~F}\right] 46$ & & & 1.3 & 1.5 & 6.5 & 2.7 & 5.0 & 1.8 \\
\hline$\left[{ }^{18} \mathrm{~F}\right] 47$ & & & 4.0 & 0.7 & 3.1 & 0.9 & 0.8 & 1.3 \\
\hline
\end{tabular}

${ }^{\text {a) }}$ Biodistribution studies in wild-type mice were performed in duplicate or triplicate: organs of interest were collected at 5 and 30 min p.i., respectively, and analyzed by gamma-counting; average radioactivity uptake given as \% injected dose per gram bodyweight (\% ID/g). 


\section{FIGURE LEGENDS}

Figure 1. Illustration of the study design. A) Structures of hydroxyzine and terfenadine, which were used as templates for the development of the pro-drug tracers, and their oxidative metabolism to cetirizine and fexofenadine, respectively; B) Depiction of the structural modifications that were investigated in this study.

Figure 2. Fluorinated non-radioactive analogues of potential pro-drug tracers.

Figure 3. Stimulation of P-gp ATPase activity by selected compounds. Reduction of luminescence in counts per minute; all compounds except verapamil $(200 \mu \mathrm{M})$ were tested at a concentration of $20 \mu \mathrm{M}$; experiments were performed in triplicate; abbreviations: Cet, cetirizine; Con, negative control sodium orthovanadate; Fex, fexofenadine; Ver, positive control verapamil.

Figure 4. Organ distribution and metabolic profile of $\left[{ }^{18} \mathbf{F}\right] 40$ and $\left[{ }^{18} \mathbf{F}\right] 44$ in wild type FVB mice $(n=3)$. A) Blood and brain uptake of $\left[{ }^{\mathbf{1 8}} \mathbf{F}\right] \mathbf{4 0}$ at 5, 15, 30, 45, 60, 90 and 120 min p.i. in \% ID/g; B) Blood and brain uptake of $\left[{ }^{18} \mathbf{F}\right] 44$ at 5, 60 and $120 \mathrm{~min}$ p.i. in \% ID/g; C/D) Metabolic profile of $\left[{ }^{18} \mathbf{F}\right] 40$ in brain and blood at 30, 60, 90 and 120 min p.i. as determined by radio-HPLC.

Figure 5. Blocking studies. A) Brain (filled columns) and blood (dotted columns) uptake of $\left[{ }^{18} \mathbf{F}\right] 40$ before (black) and after administration of tariquidar (light grey) and elacridar (dark grey) in wild type FVB mice $\left(\mathrm{n}=3 ;{ }^{*} \mathrm{p}<0.05\right)$; B) Whole brain time-activity curves from dynamic PET scans in untreated wild type mice (black circles), tariquidar-treated mice (light grey squares) and P-gp knockout mice (open triangles) (experiment performed at AIT); C) Whole brain timeactivity curves from dynamic PET scans in untreated wild type mice (black circles) and elacridar-treated mice (dark grey squares) (experiment performed at UCL). 


\section{SCHEME LEGENDS}

Scheme 1. Synthesis of simplified fexofenadine derivatives

Caption: Reagents and conditions: (i) $\mathrm{K}_{2} \mathrm{CO}_{3}, \mathrm{KI}, \mathrm{CH}_{3} \mathrm{CN}, 80{ }^{\circ} \mathrm{C}, 12 \mathrm{~h}$; or: $\mathrm{K}_{2} \mathrm{CO}_{3}$, acetone, 60 ${ }^{\circ} \mathrm{C}-\mathrm{rt}, 12 \mathrm{~h}$; (ii) oxalyl chloride, DMSO, TEA, DCM, <-50 ${ }^{\circ} \mathrm{C}, 30 \mathrm{~min}$; (iii) $\mathrm{KOH}, \mathrm{H}_{2} \mathrm{O}$ : THF: $\mathrm{CH}_{3} \mathrm{OH}=2: 1: 1, \mathrm{MW}, 70{ }^{\circ} \mathrm{C}, 15 \mathrm{~min}$.

Scheme 2. Synthesis of compounds 18-28 with an aliphatic fluorinated side chain Caption: Reagents and conditions: (i) DAST, DCM, $0{ }^{\circ} \mathrm{C}-\mathrm{rt}, 3 \mathrm{~h}$; (ii) $\mathrm{NaOCH}_{3}, \mathrm{CH}_{3} \mathrm{OH}$, rt, 1 h; (iii) TsCl, pyridine, DCM, $0{ }^{\circ} \mathrm{C}-\mathrm{rt}, 12 \mathrm{~h}$; (iv) 1-benzhydryl-piperazine (A)/diphenylpiperidin-4-yl-methanol (B), TEA, $\mathrm{CH}_{3} \mathrm{CN}, 70{ }^{\circ} \mathrm{C}, 6 \mathrm{~h}$; (v) $\mathrm{LiAlH}_{4}$, THF, $0{ }^{\circ} \mathrm{C}-\mathrm{rt}, 1 \mathrm{~h}$; (vi) $\mathrm{KOH}, \mathrm{H}_{2} \mathrm{O}$ : THF: $\mathrm{CH}_{3} \mathrm{OH}=2: 1: 1, \mathrm{rt}, 1$ h; (vii) $\mathrm{Ac}_{2} \mathrm{O}$, TEA, DCM, rt, 3 h.

Scheme 3. Synthesis of the fluorinated scaffolds C-F

Caption: Reagents and conditions: (i) PCC, celite, DCM, rt, 3 h; (ii) 4F-PhMgBr, THF, 0 - 60 ${ }^{\circ} \mathrm{C}, 2$ h; (iii) Dess-Martin periodinane, DCM, rt, 2 h; (iv) $\mathrm{PhMgBr}$, THF, $0-60{ }^{\circ} \mathrm{C}, 1 \mathrm{~h}$; (v) $\mathrm{HCl}$, dioxane, THF, rt, 12 h; (vi) TFA, DCM, rt, 3 h.

Scheme 4. Synthesis of precursors for radiolabeling of aliphatic residues

Caption: Reagents and conditions: (i) TsCl, pyridine, DCM, $0^{\circ} \mathrm{C}-\mathrm{rt}, 12 \mathrm{~h}$; (ii) 1-benzhydrylpiperazine (a)/diphenyl-piperidin-4-yl-methanol (b), TEA, $\mathrm{CH}_{3} \mathrm{CN}, 70{ }^{\circ} \mathrm{C}, 6 \mathrm{~h}$; (iii) PTSA, $\mathrm{CH}_{3} \mathrm{OH}, 40{ }^{\circ} \mathrm{C}, 4 \mathrm{~h}$; (iv) $\mathrm{Ac}_{2} \mathrm{O}$, TEA, DCM, $0{ }^{\circ} \mathrm{C}-\mathrm{rt}, 3 \mathrm{~h}$; (v) MsCl, TEA, DCM, rt, 1 h.

Scheme 5. Synthesis of triarylsulfonium triflates as precursors for labeling with fluoride-18

Caption: Reagents and conditions: (i) $1 . n$-BuLi, THF, $-78{ }^{\circ} \mathrm{C}, 30 \mathrm{~min} ; 2 . \mathbf{5 8}$, THF, $-78{ }^{\circ} \mathrm{C}-0$ ${ }^{\circ} \mathrm{C}, 30 \mathrm{~min}$; (ii) $\mathrm{HCl}$, THF, rt, $12 \mathrm{~h}$; (iii) bromoalkane, $\mathrm{K}_{2} \mathrm{CO}_{3}$, acetone, $60{ }^{\circ} \mathrm{C}-\mathrm{rt}, 12 \mathrm{~h}$; (iv) (Ph) 
${ }_{2} \mathrm{I}^{+} \cdot \mathrm{CF}_{3} \mathrm{O}_{3} \mathrm{~S}^{-}, \mathrm{Cu}(\mathrm{II})$ benzoate, $\mathrm{CHF}_{3} \mathrm{O}_{3} \mathrm{~S}, \mathrm{C}_{6} \mathrm{H}_{5} \mathrm{Cl}, 125^{\circ} \mathrm{C}, 1 \mathrm{~h}$; (v) PhMgBr, THF, $0{ }^{\circ} \mathrm{C}-\mathrm{rt}, 3 \mathrm{~h}$;

(vi) TFA, DCM, $0{ }^{\circ} \mathrm{C}-\mathrm{rt}, 2 \mathrm{~h}$; (vii) $\left(4-\mathrm{OCH}_{3}-\mathrm{Ph}\right)_{2} \mathrm{I}^{+} \cdot \mathrm{CF}_{3} \mathrm{O}_{3} \mathrm{~S}^{-}(\mathbf{7 5}), \mathrm{Cu}$ (II) benzoate, $\mathrm{CHF}_{3} \mathrm{O}_{3} \mathrm{~S}$, $\mathrm{C}_{6} \mathrm{H}_{5} \mathrm{Cl}, 125^{\circ} \mathrm{C}, 1 \mathrm{~h}$.

Scheme 6. Radiosynthesis of tracers $\left[{ }^{18} \mathbf{F}\right] 23$ and $\left[{ }^{18} \mathbf{F}\right] 27$

Caption: Reagents and conditions: (i) $\left[{ }^{18} \mathrm{~F}^{\mathrm{F}} \mathrm{F}^{-}, \mathrm{K}_{2} \mathrm{CO}_{3}, \mathrm{~K}_{222}\right.$, DMSO, $90{ }^{\circ} \mathrm{C}, 20 \mathrm{~min}$.

Scheme 7. Radiosynthesis of ketanserin- and fexofenadine-like pro-drug tracer candidates

Caption: Reagents and conditions: (i) $\left[{ }^{18} \mathrm{~F}^{-} \mathrm{F}^{-}, \mathrm{KHCO}_{3}, \mathrm{~K}_{222}, \mathrm{DMSO}, 110{ }^{\circ} \mathrm{C}, 15 \mathrm{~min}\right.$; (ii) $\mathrm{HCl}$, $\mathrm{C}_{2} \mathrm{H}_{5} \mathrm{OH}, 90^{\circ} \mathrm{C}, 15 \mathrm{~min}$; (iii) $\mathrm{PhMgBr}$, THF, $90{ }^{\circ} \mathrm{C}, 30 \mathrm{~min}$; (iv) $\mathrm{AcCl}, \mathrm{CH}_{3} \mathrm{CN}, \mathrm{rt}, 15 \mathrm{~min}$.

Scheme 8. Radiosynthesis of ritanserin-like pro-drug tracer candidates

Caption: Reagents and conditions: (i) $\left[{ }^{18} \mathrm{~F}^{-} \mathrm{F}^{-}, \mathrm{KHCO}_{3}, \mathrm{~K}_{222}, \mathrm{DMSO}, 110^{\circ} \mathrm{C}, 15 \mathrm{~min}\right.$; (ii) $\mathrm{HCl}$, $\mathrm{C}_{2} \mathrm{H}_{5} \mathrm{OH}, 90{ }^{\circ} \mathrm{C}, 15 \mathrm{~min}$; (iii) $\mathrm{NaOH}, \mathrm{C}_{2} \mathrm{H}_{5} \mathrm{OH}, 90{ }^{\circ} \mathrm{C}, 15 \mathrm{~min}$; (iv) $\mathrm{HCl}$, THF, $90{ }^{\circ} \mathrm{C}, 15 \mathrm{~min}$. 


\section{FIGURE 1}

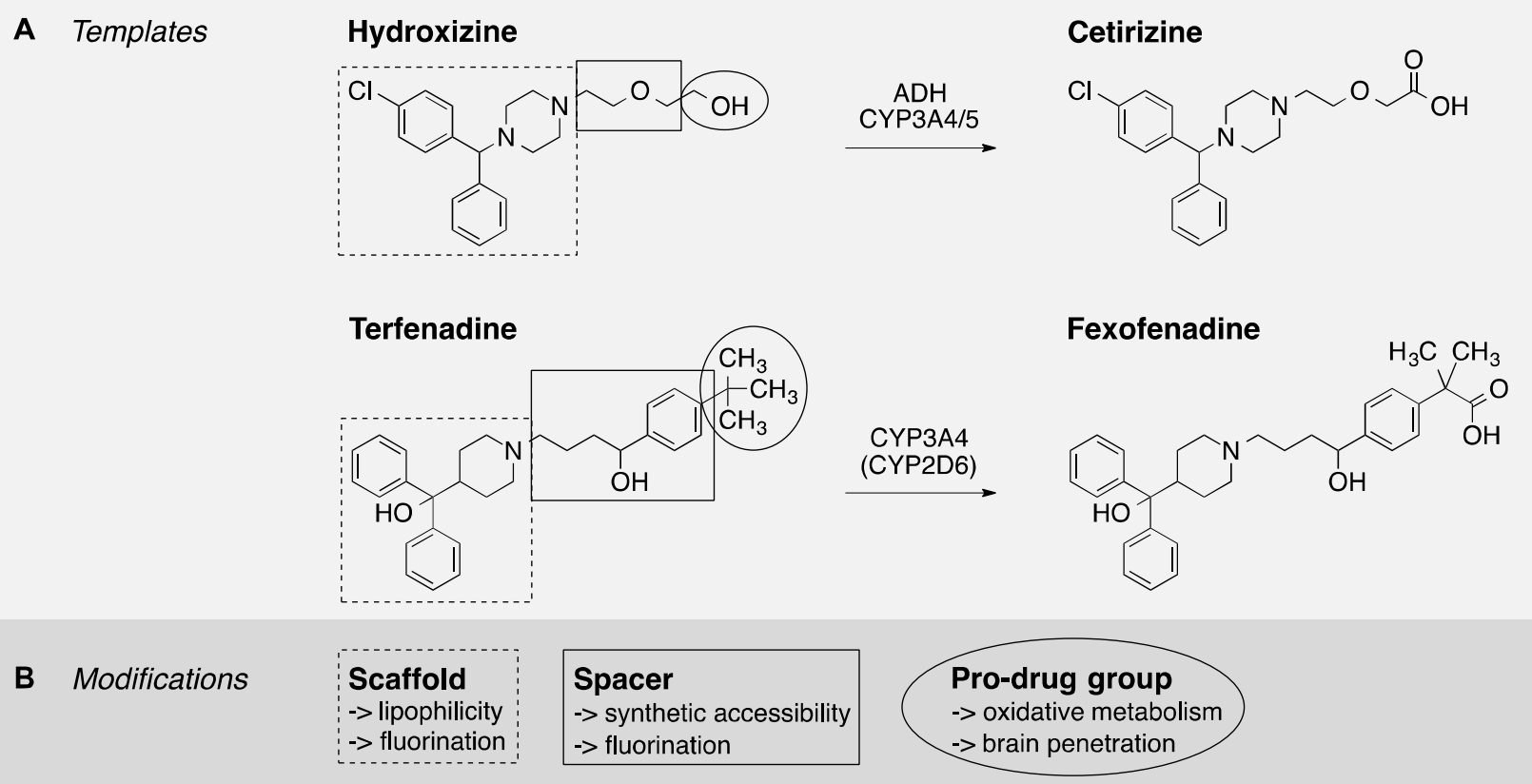

\section{drug}

idative metabolism

$>$ brain penetration 


\section{FIGURE 2}

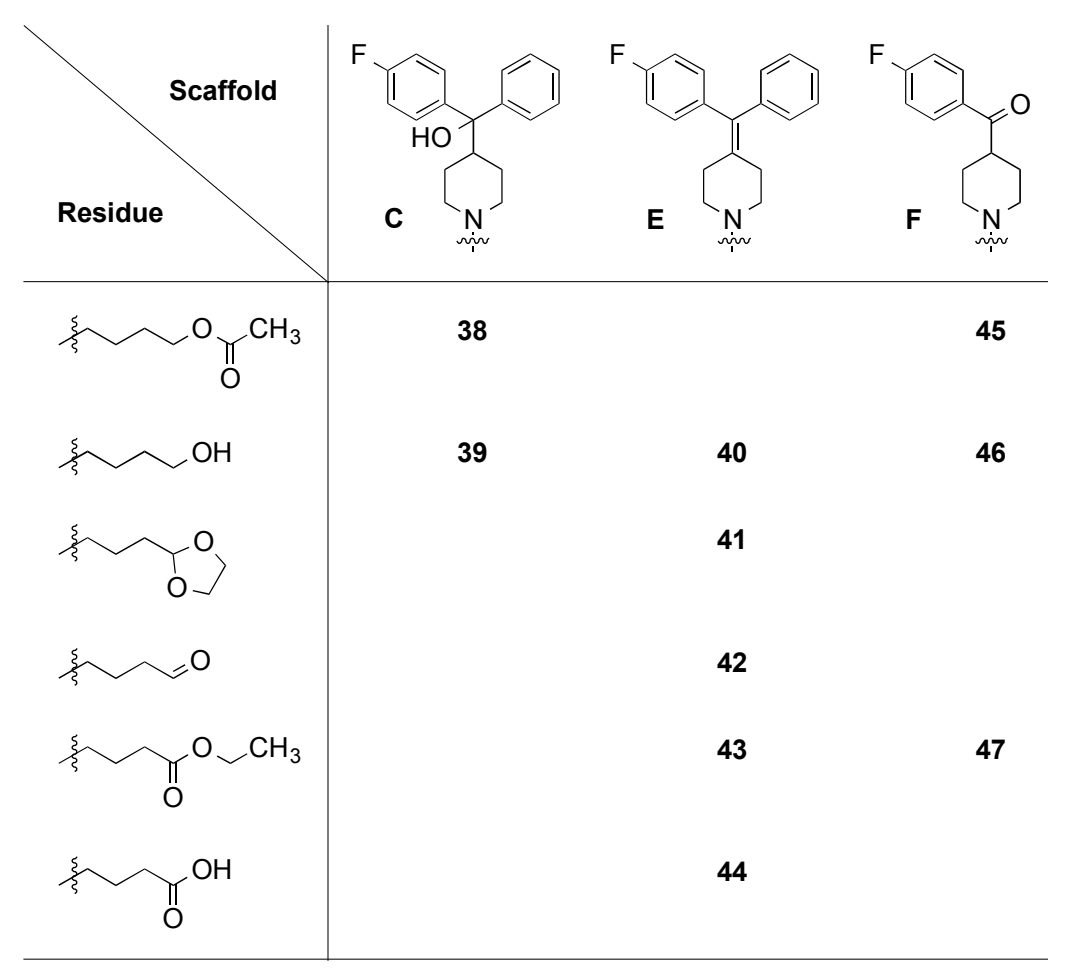

30

31

32

33

34

35

36

37

38

39

40

41

42

43

44

45

46

47

48

49

50

51

52

53

54

55

56

57

58

59

60 


\section{FIGURE 3}

A<smiles>[3H]Cc1ccc(C(=O)O)cc1</smiles>

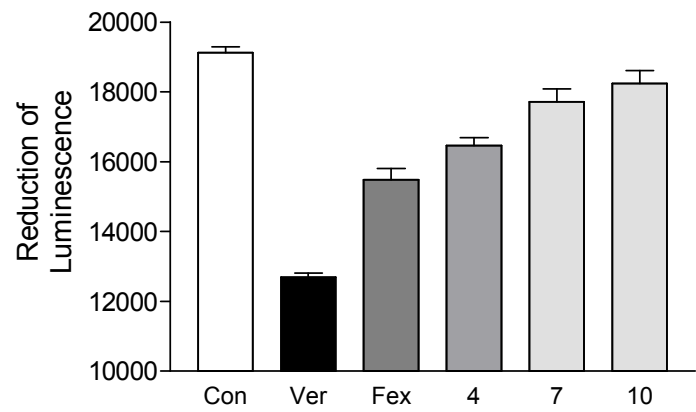

C
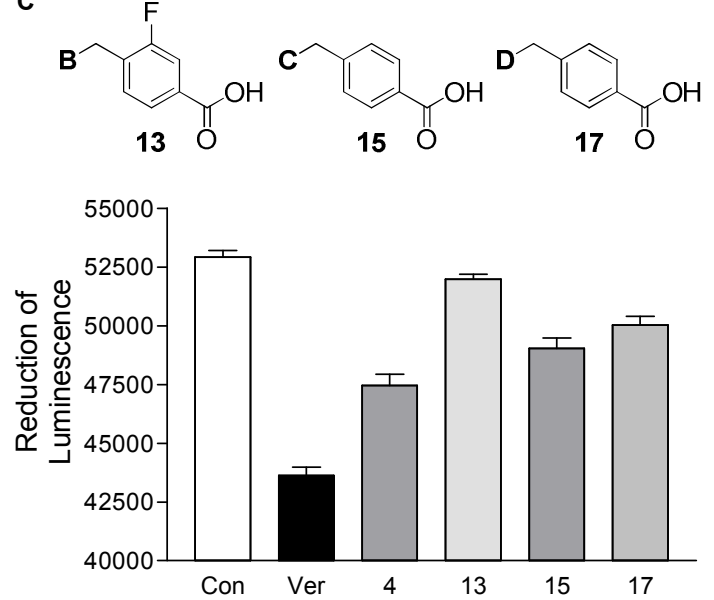

B<smiles>[B]Cc1ccc(CO)cc1</smiles>

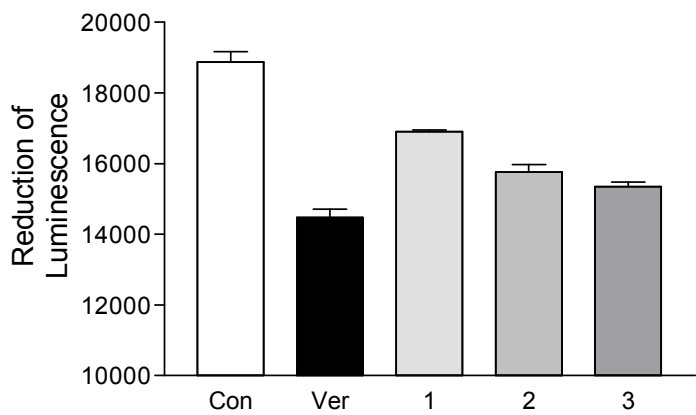

D<smiles>[Y]CCC(F)CO</smiles>

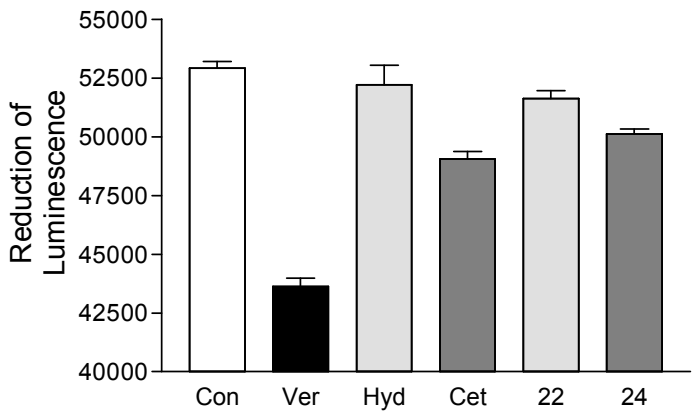

Scaffolds<smiles>C[NH+]1CCN(C(c2ccccc2)c2ccccc2)CC1</smiles><smiles>[B]C(c1ccccc1)(c1ccccc1)C1CC[NH+](C)CC1</smiles><smiles>CN1CCC(C(O)(c2ccccc2)c2ccc(F)cc2)CC1</smiles><smiles>CN1CCC(C(O)(c2ccc(F)cc2)c2ccc(F)cc2)CC1</smiles> 
1

2

3

4

5

6

7

8

9

10

11

12

13

14

15

16

17

18

19

20

21

22

23

24

25

26

27

28

29

30

31

32

33

34

35

36

37

38

39

40

41

42

43

44

45

46

47

48

49

50

51

52

53

54

55

56

57

58

59

60

\section{FIGURE 4}

A

\% ID/g

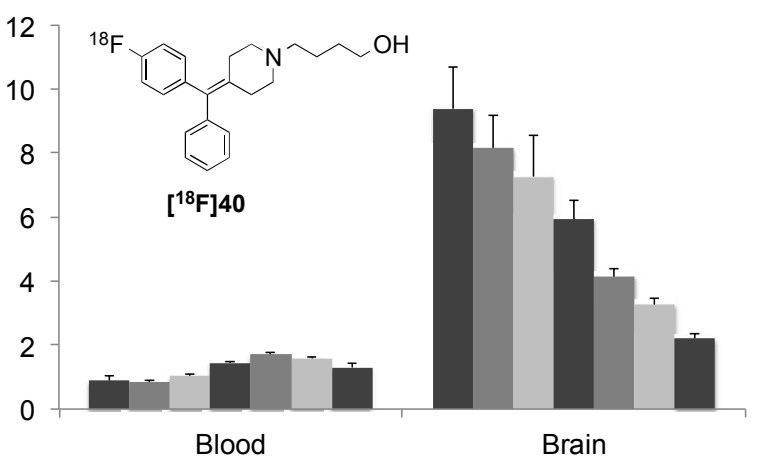

C

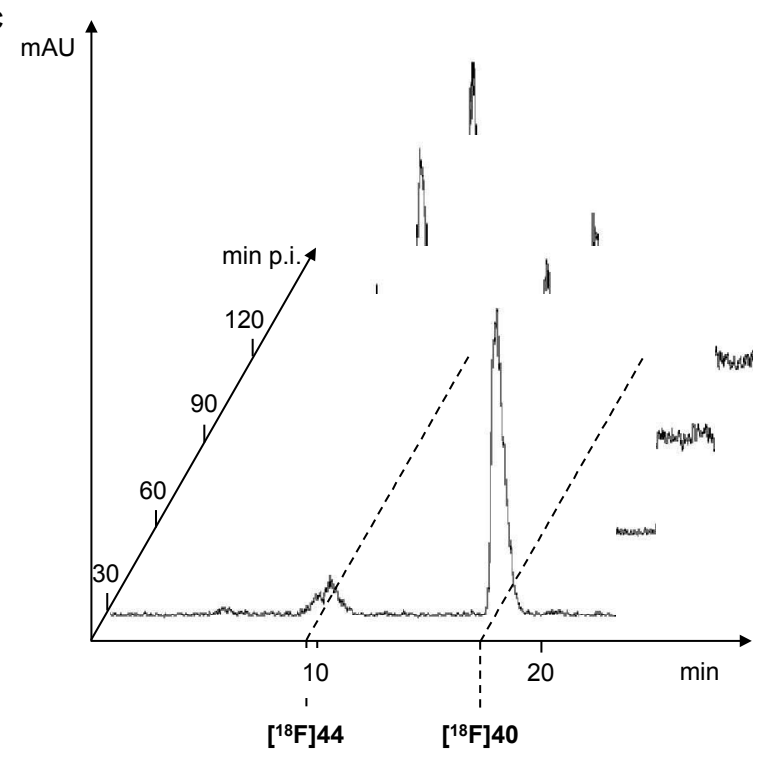

B

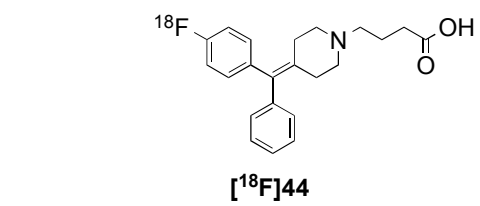

\% ID/g 4
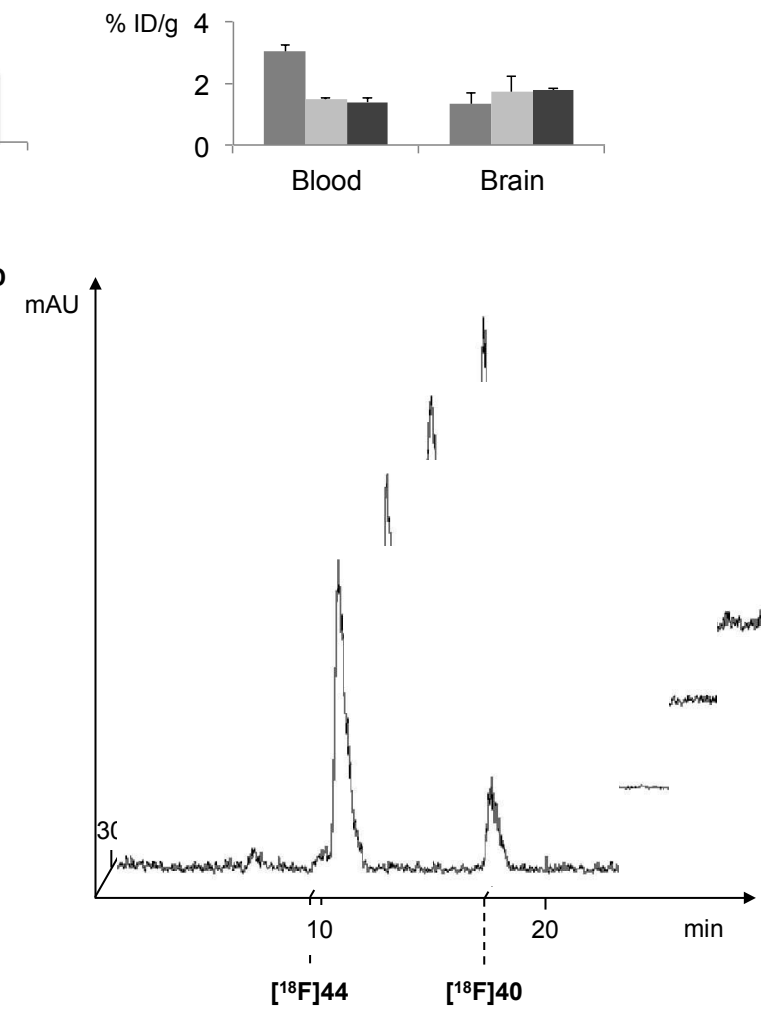


\section{FIGURE 5}

A

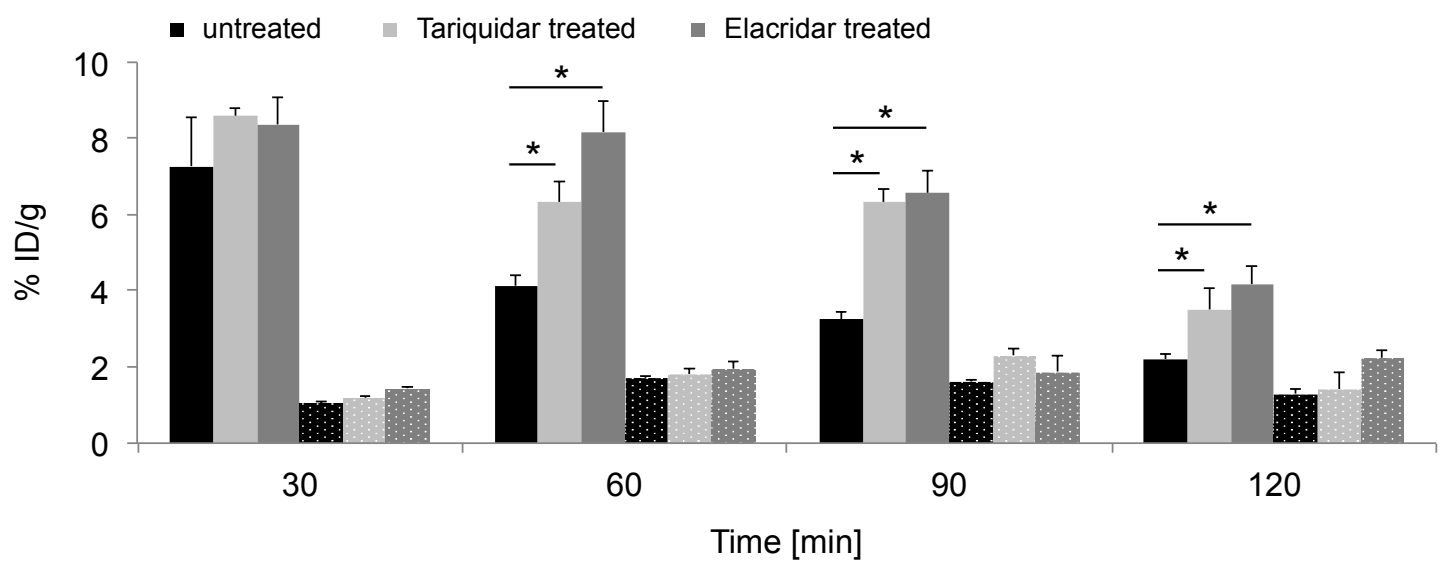

B

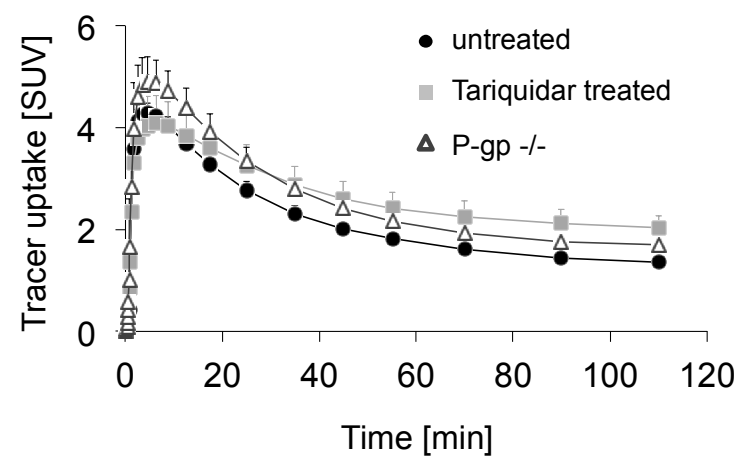

\begin{tabular}{l|ll} 
Group & $\begin{array}{l}\text { Efflux rate } \\
(1 / \mathrm{min})\end{array}$ & $\begin{array}{l}\text { Radioactivity: } \\
\text { washout / trapped }\end{array}$ \\
\hline Control & 0.0378 & 2.89 \\
Tariquidar & 0.0333 & 1.50 \\
P-gp -/- & 0.0387 & 2.77
\end{tabular}

C

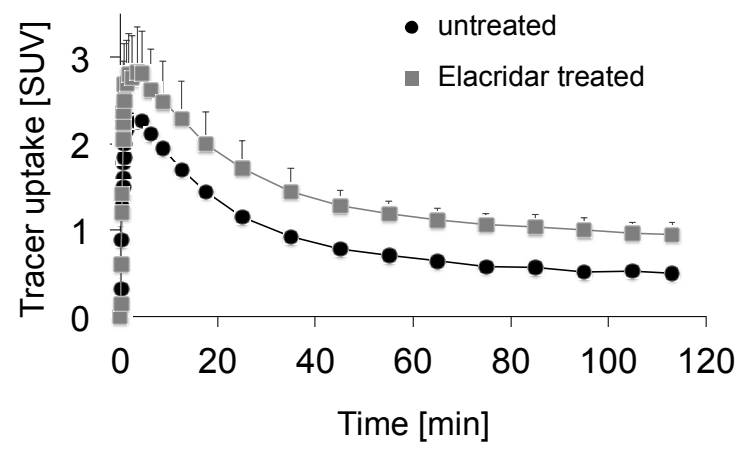

\begin{tabular}{l|ll} 
Group & $\begin{array}{l}\text { Efflux rate } \\
(1 / \mathrm{min})\end{array}$ & $\begin{array}{l}\text { Radioactivity: } \\
\text { washout / trapped }\end{array}$ \\
\hline Control & 0.0454 & 4.14 \\
Elacridar & 0.0432 & 2.33
\end{tabular}


SCHEME 1
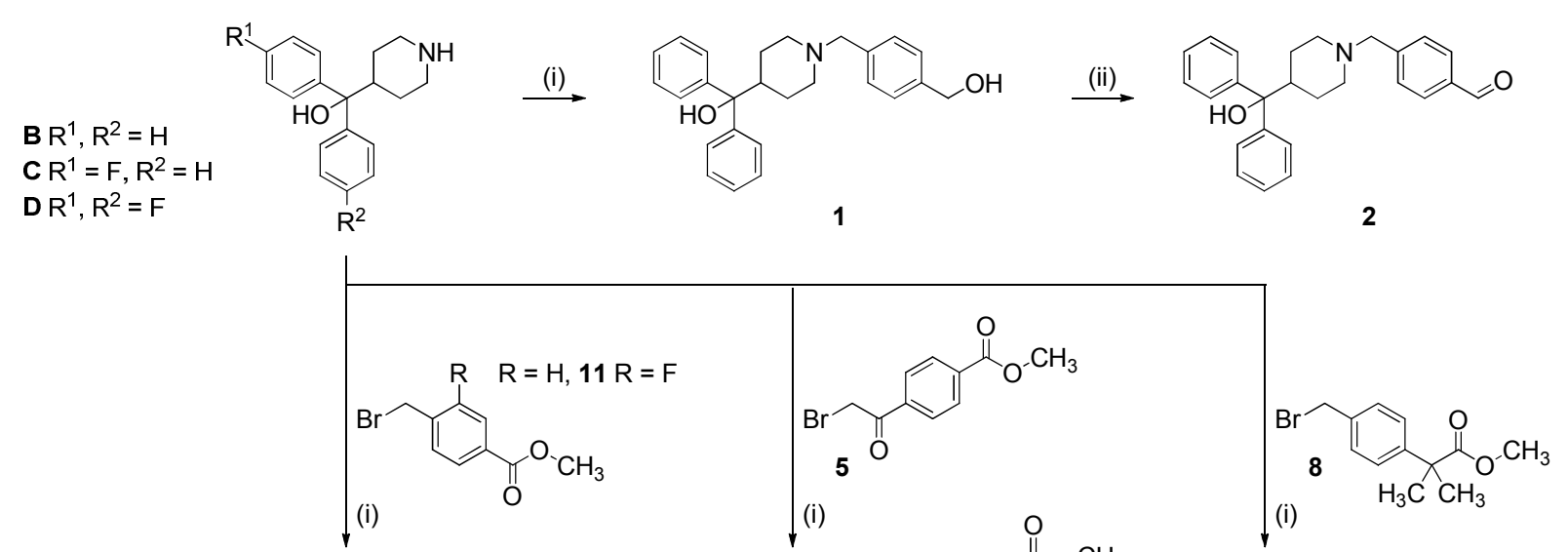

$3 \mathrm{R}^{1}, \mathrm{R}^{2}, \mathrm{R}=\mathrm{H}$ $12 R^{1}, R^{2}=H, R=F$ $14 R^{1}=F, R^{2}, R=H$ $16 R^{1}, R^{2}=F, R=H$

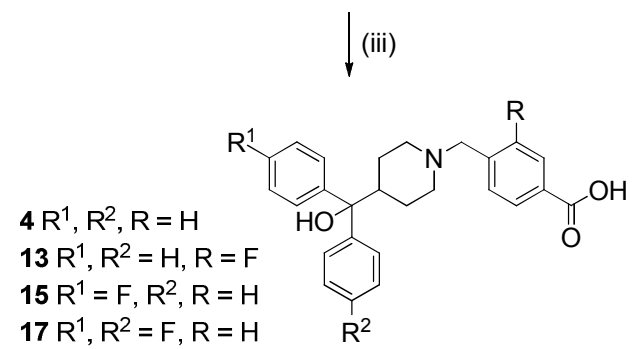<smiles>[R]c1ccc(C(O)(c2ccc([R4])cc2)C2CCN(Cc3ccc(C(=O)OC)cc3[R])CC2)cc1</smiles><smiles>COC(=O)c1ccc(C(=O)CN2CCC(C(O)(c3ccccc3)c3ccccc3)CC2)cc1</smiles>

6

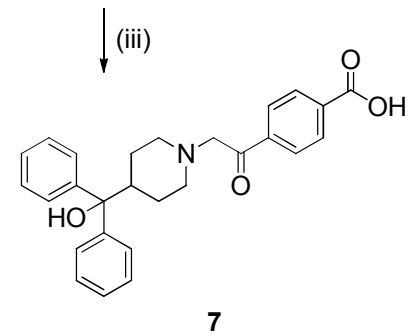

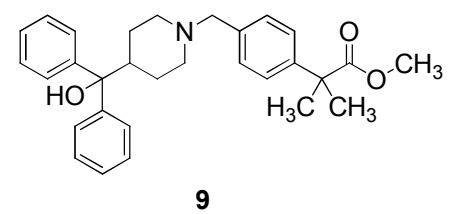

$\downarrow$ (iii)

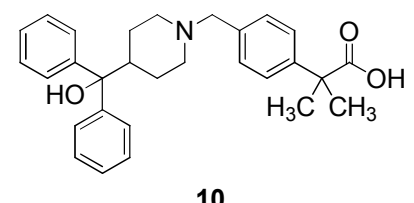




\section{SCHEME 2}

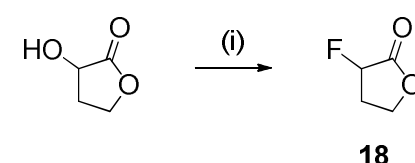<smiles>C#CC#CC</smiles><smiles>COC(=O)C(F)CCOS(=O)(=O)c1ccc(C)cc1</smiles>

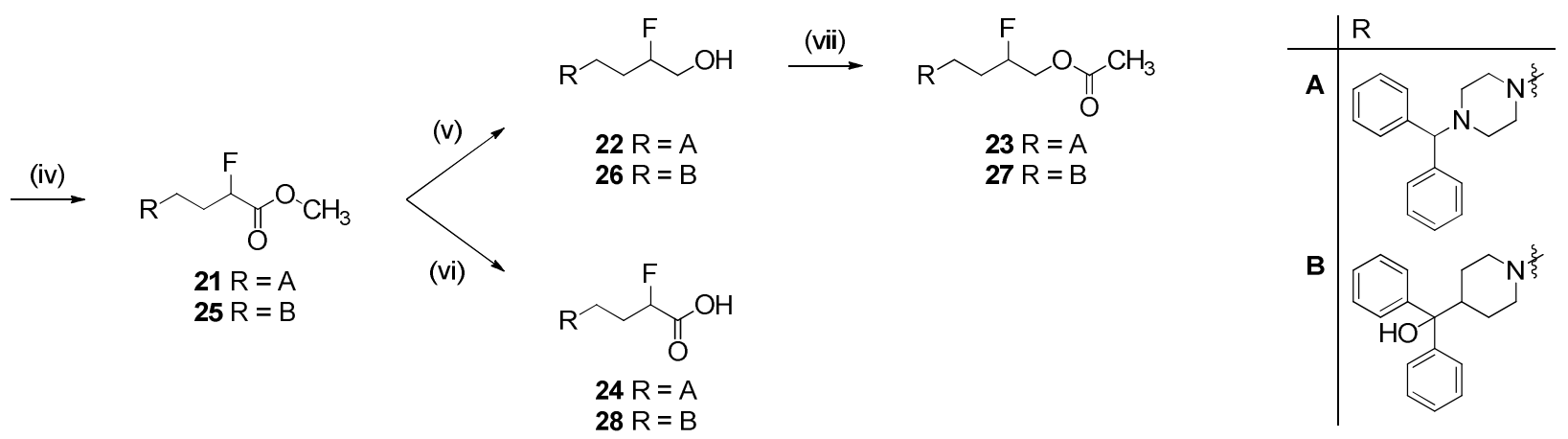




\section{SCHEME 3}
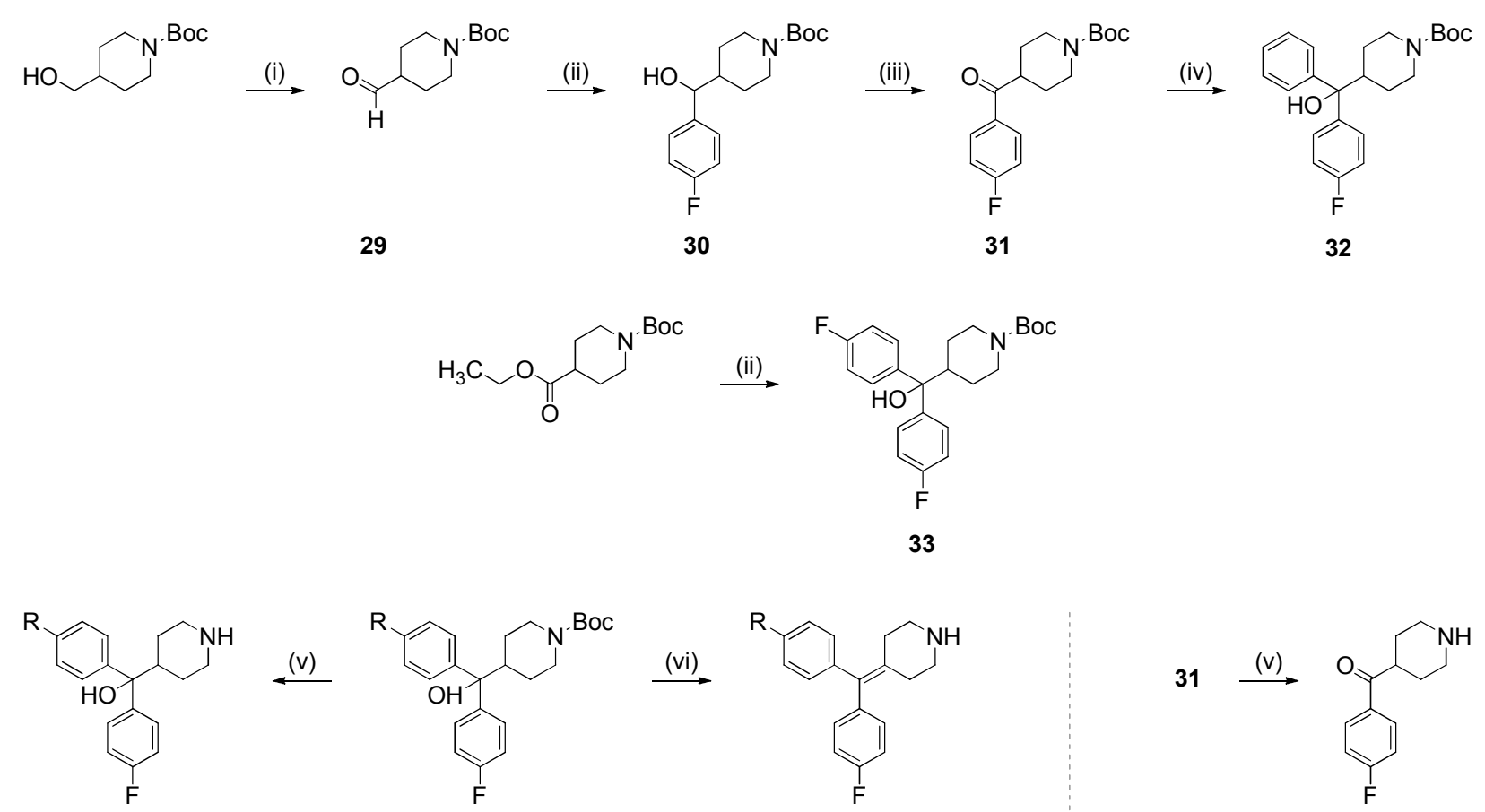

34 (C) $\mathrm{R}=\mathrm{H}$

35 (D) $R=F$

$32 \mathrm{R}=\mathrm{H}$
$33 \mathrm{R}=\mathrm{F}$

$36(E) R=H$

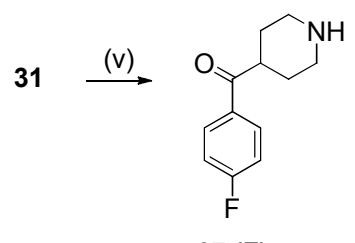

30

31

32

33

34

35

36

37

38

39

40

41

42

43

44

45

46

47

48

49

50

51

52

53

54

55

56

57

58

59

60 


\section{SCHEME 4}

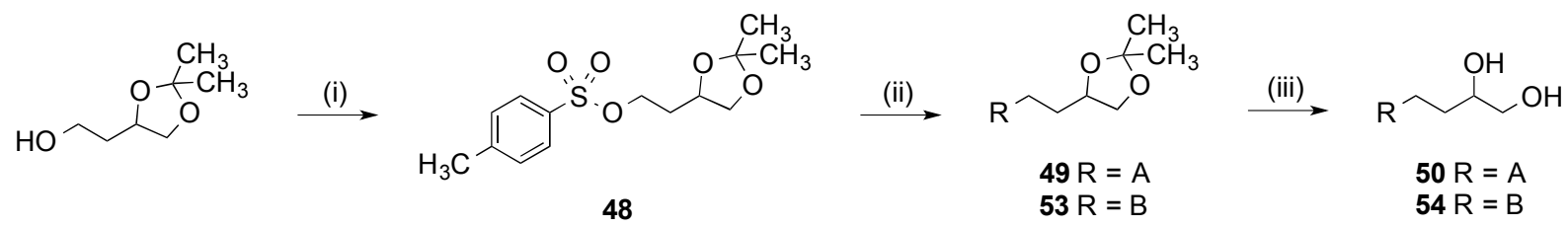<smiles>[R]CCC(O)COC(C)=O</smiles> 


\section{SCHEME 5}

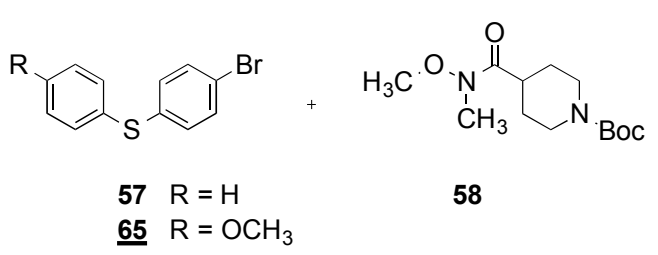

(i)

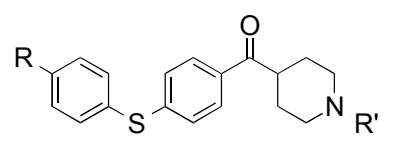
(ii) $C 59 \mathrm{R}=\mathrm{H}, \mathrm{R}^{\prime}=\mathrm{Boc}$
$60 \mathrm{R}=\mathrm{H}, \mathrm{R}^{\prime}=\mathrm{H}$
66 $\mathrm{R}=\mathrm{OCH}_{3}, \mathrm{R}^{\prime}=\mathrm{Boc}$
(iii) $\longrightarrow \quad 61,63$

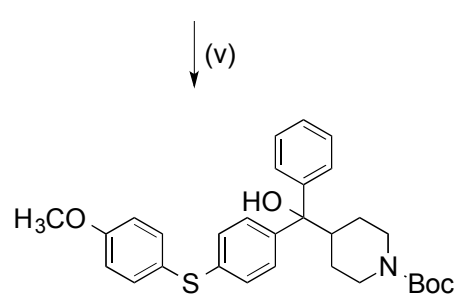

$\underline{67}$ $\downarrow$ (v)

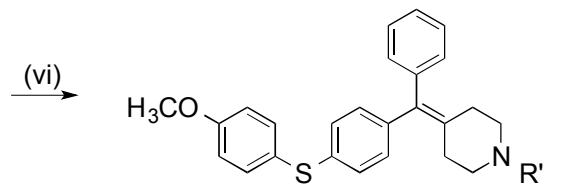

(iii) $\left(\begin{array}{l}68 R^{\prime}=H \\ 69,71,73\end{array}\right.$
$61,62 \quad \mathrm{R}^{\prime}=$

69,70

$\begin{array}{ll}63,64 \\ 71,72\end{array} \mathrm{R}^{\prime}=$

$73,74 \mathrm{R}^{\prime}=$ $\stackrel{\text { (iv) }}{\longrightarrow}$

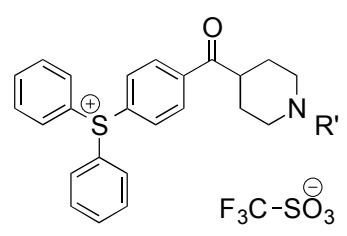

62,64

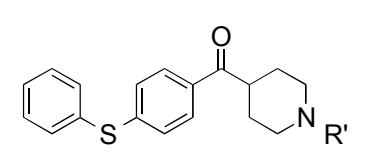

(iv)

(vii)

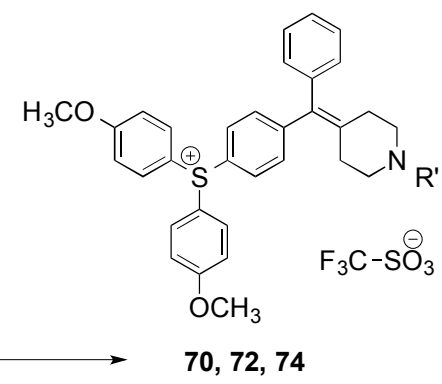

36

39

40

41

42

43

44

45

46

47

48

49

50

51

52

53

54

55

56

57

58

59

60 


\section{SCHEME 6}
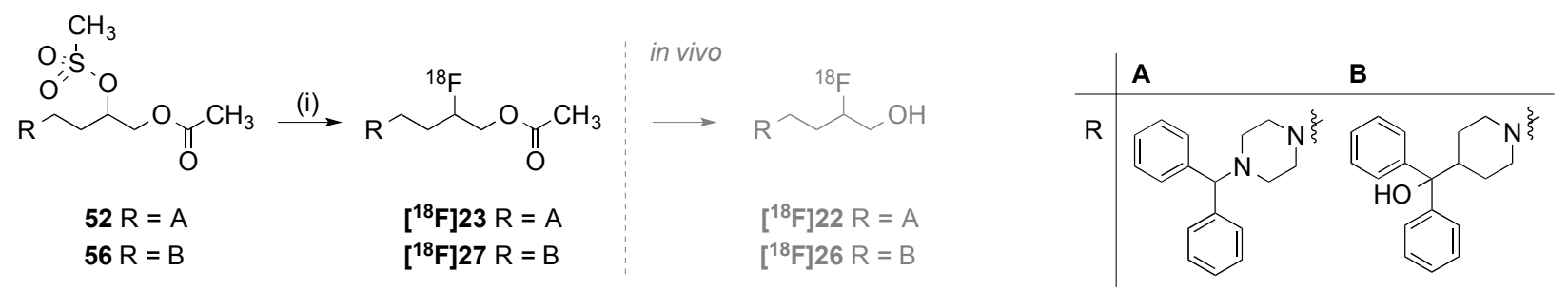


\section{SCHEME 7}

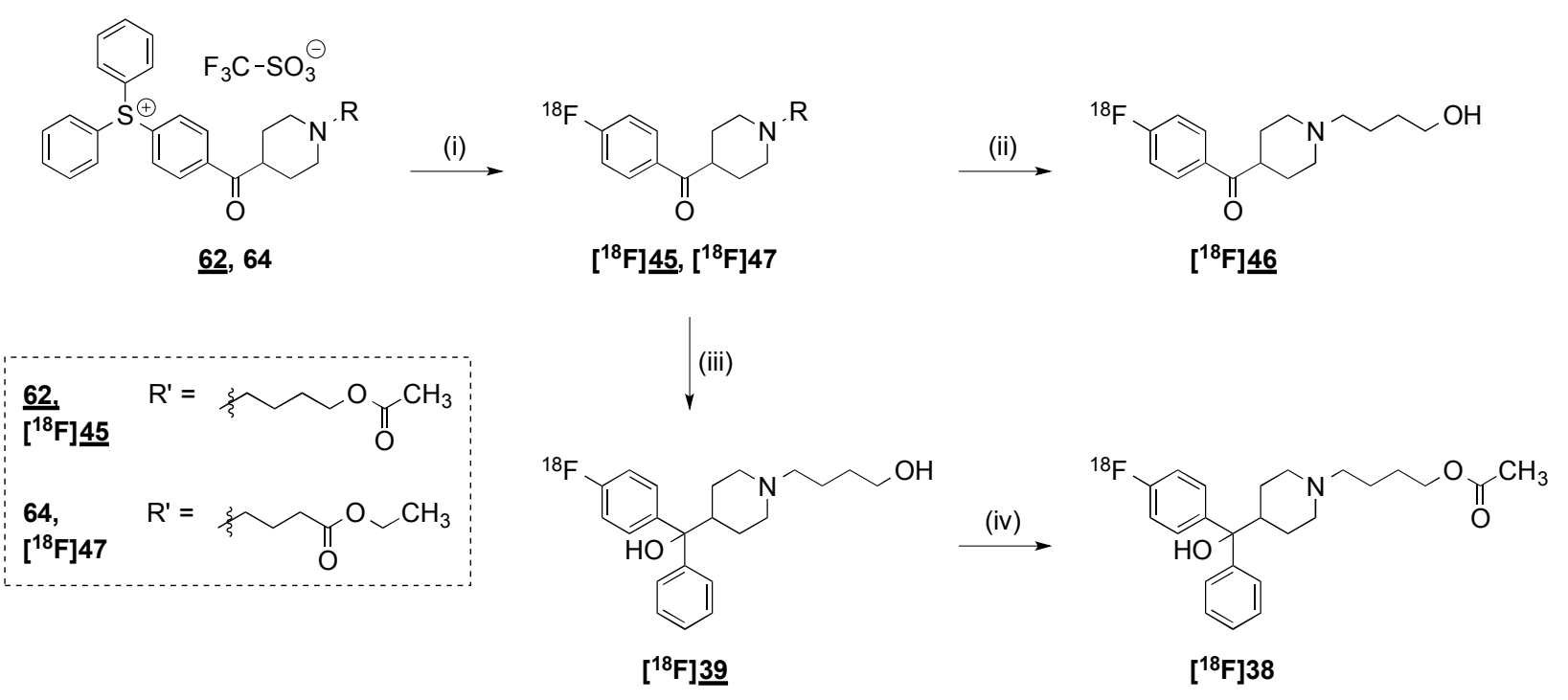




\section{SCHEME 8}

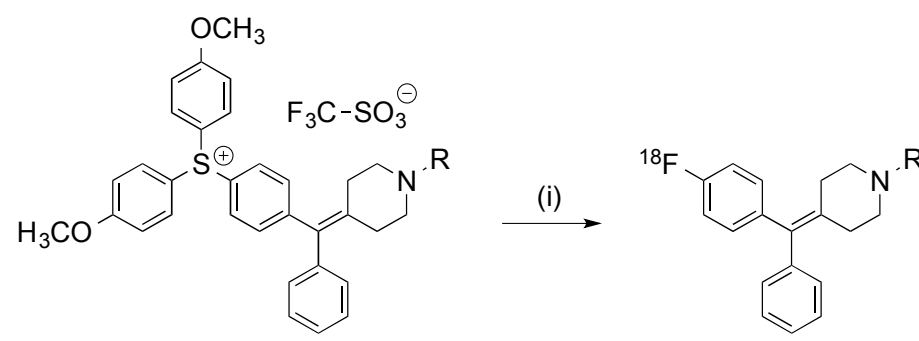

$$
\text { 70, }\left[{ }^{18} \mathrm{~F}\right] 40 \mathrm{a} \quad \mathrm{R}=\text { - }
$$




\section{TABLE OF CONTENTS GRAPHIC}

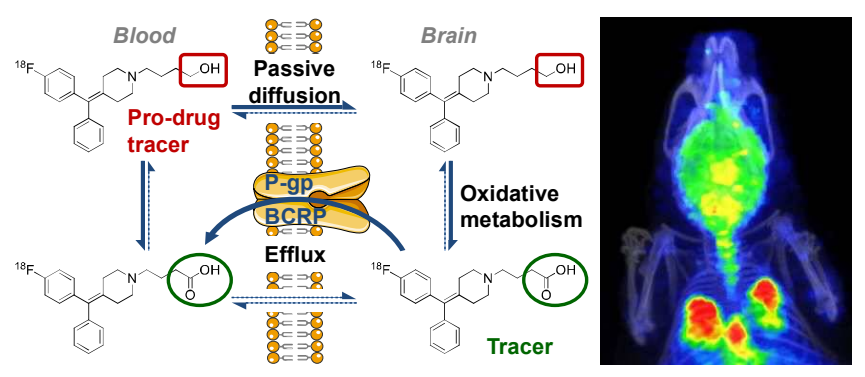

16

17

18

19

20

21

22

23

24

25

26

27

28

29

30

31

32

33

34

35

36

37

38

39

40

41

42

43

44

45

46

47

48

49

50

51

52

53

54

55

56

57

58

59

60 
A Templates

Hydroxizine

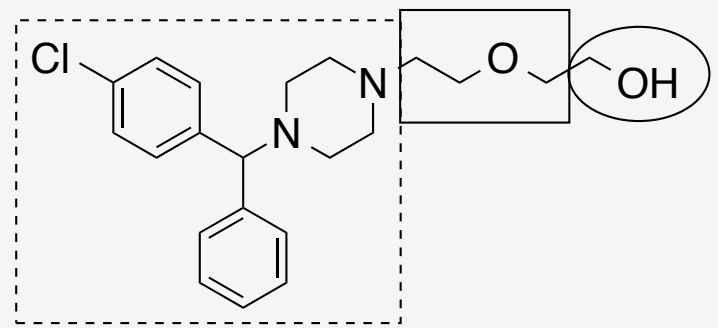

Terfenadine

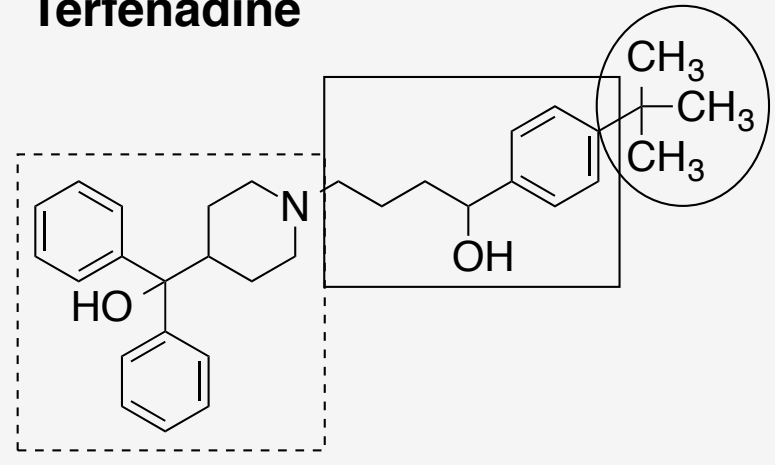

\section{Cetirizine}

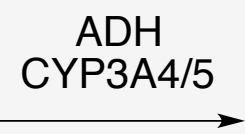

CYP3A4

(CYP2D6)<smiles>O=C(O)COCCN1CCN(C(c2ccccc2)c2ccc(Cl)cc2)CC1</smiles>

Fexofenadine

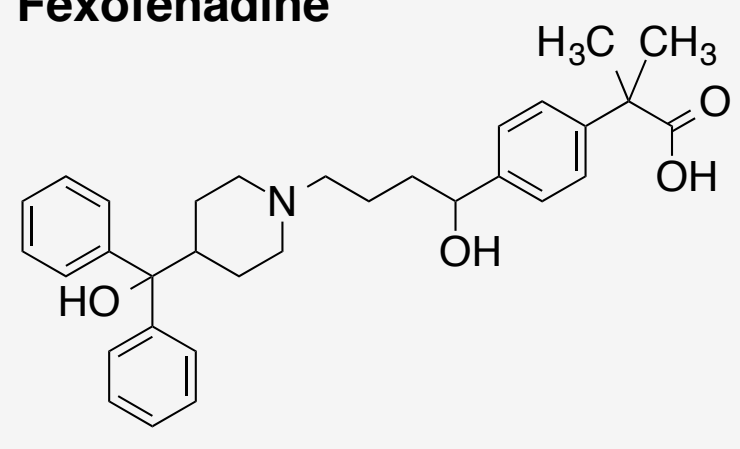

Modifications

Scaffold

-> lipophilicity

-> fluorination

\section{Spacer}

-> synthetic accessibility

$\rightarrow$ fluorination

\section{Pro-drug group}

$->$ oxidative metabolism

$\rightarrow$ brain penetration 


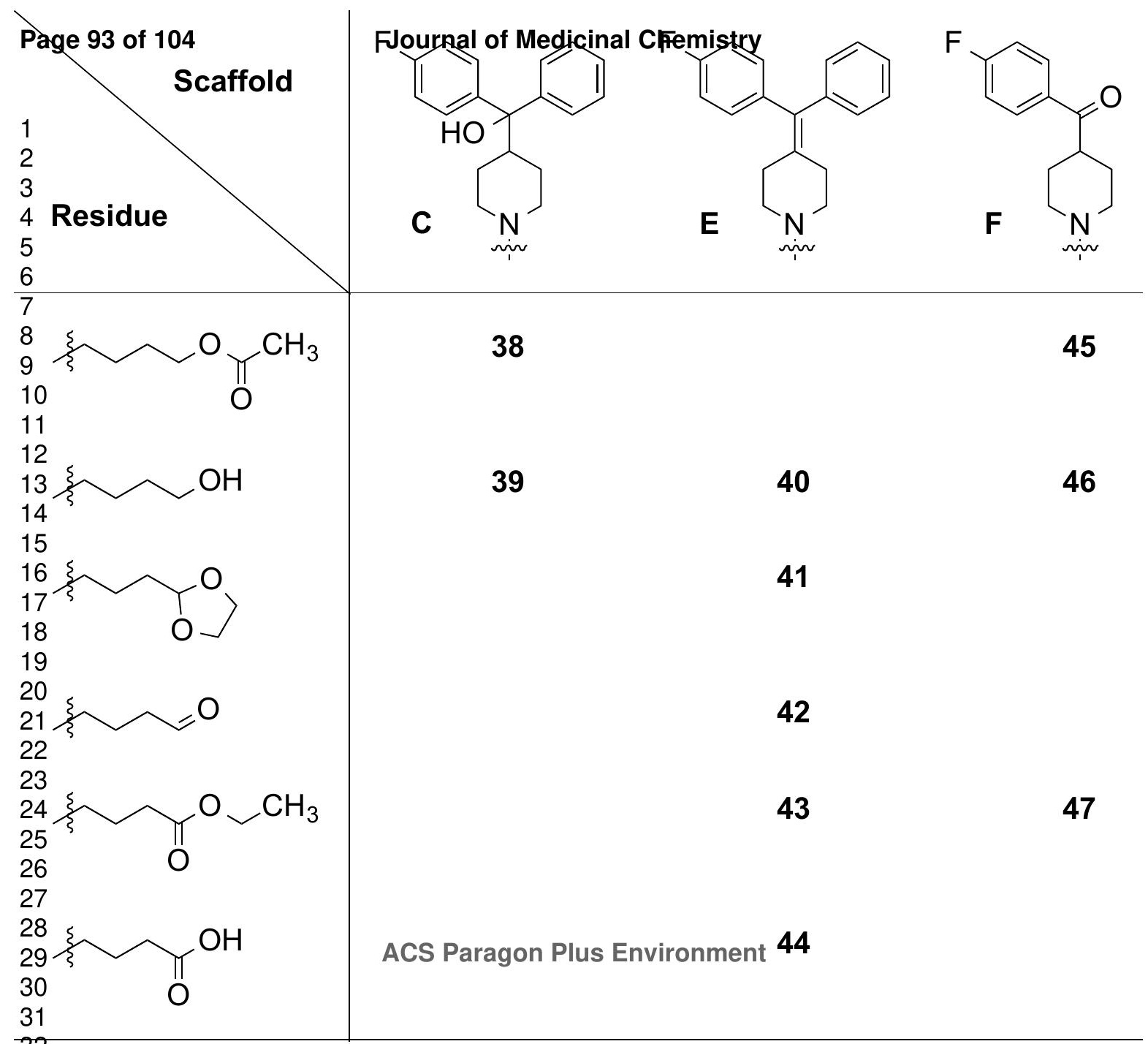




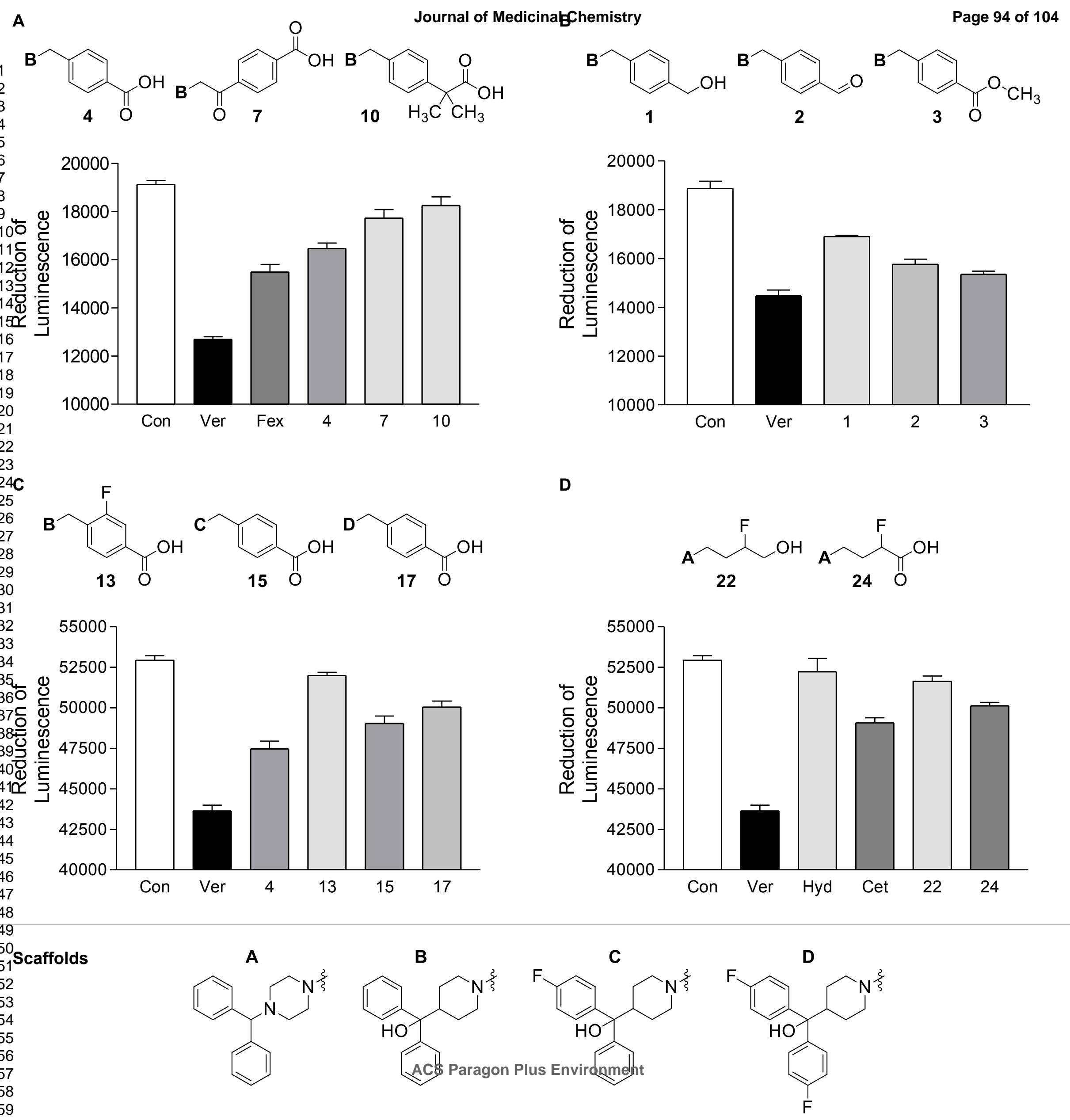




\section{Page 95 of 104 \\ stry}
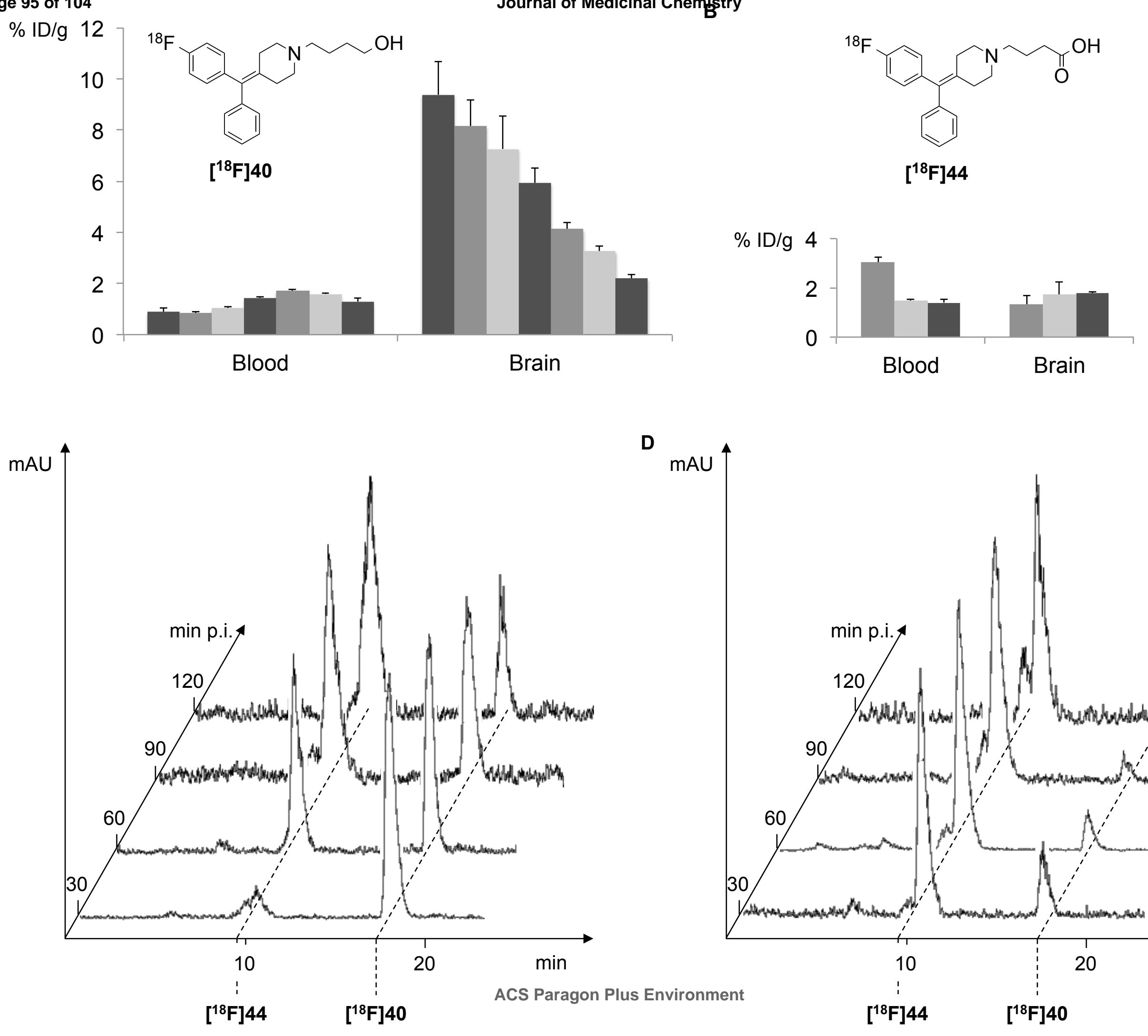

D

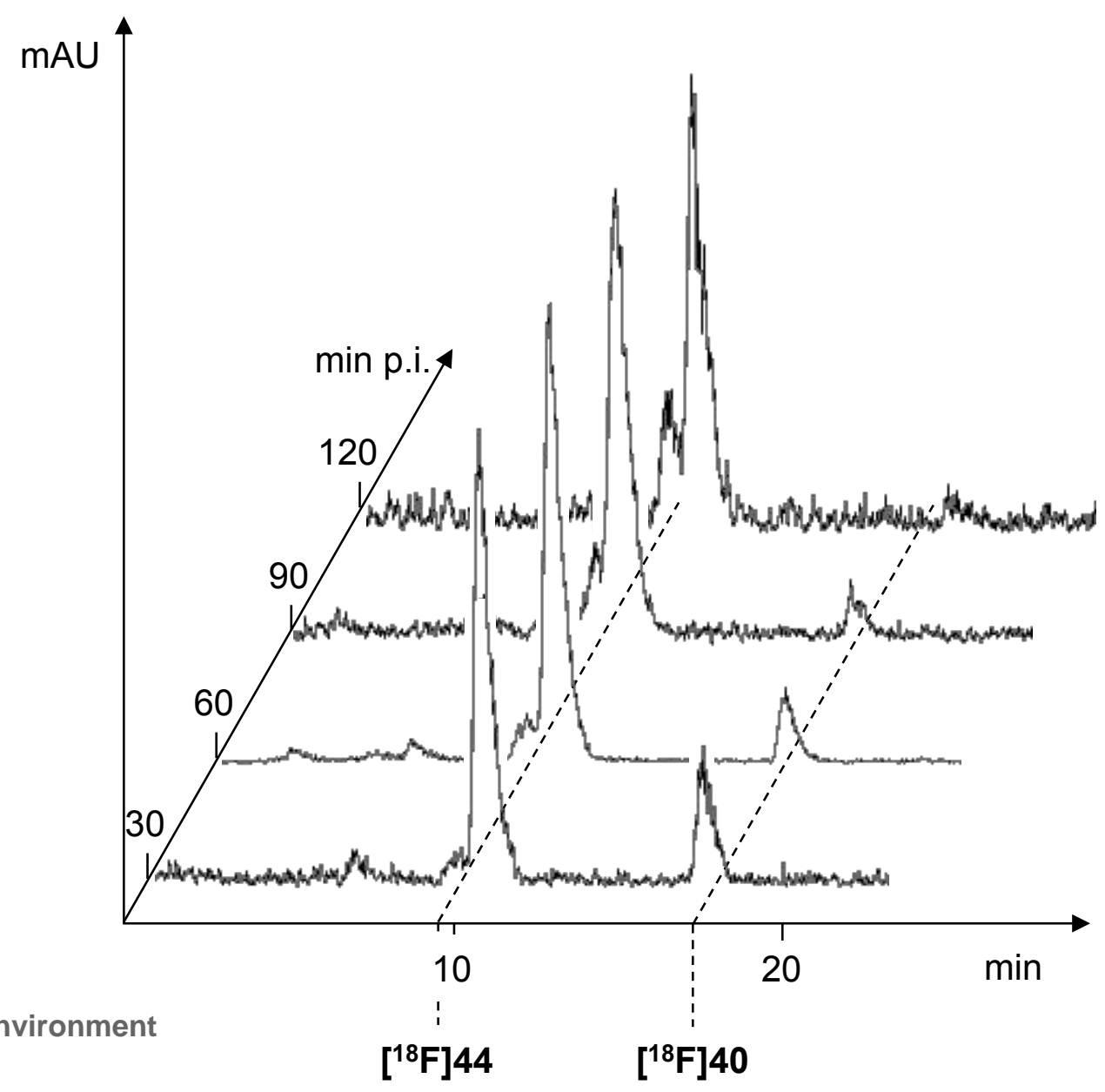

52 
A

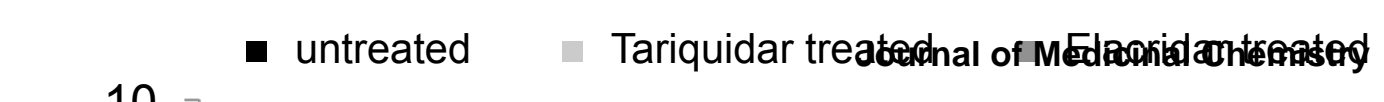

- untreated

10

1

3

5

5

7

9

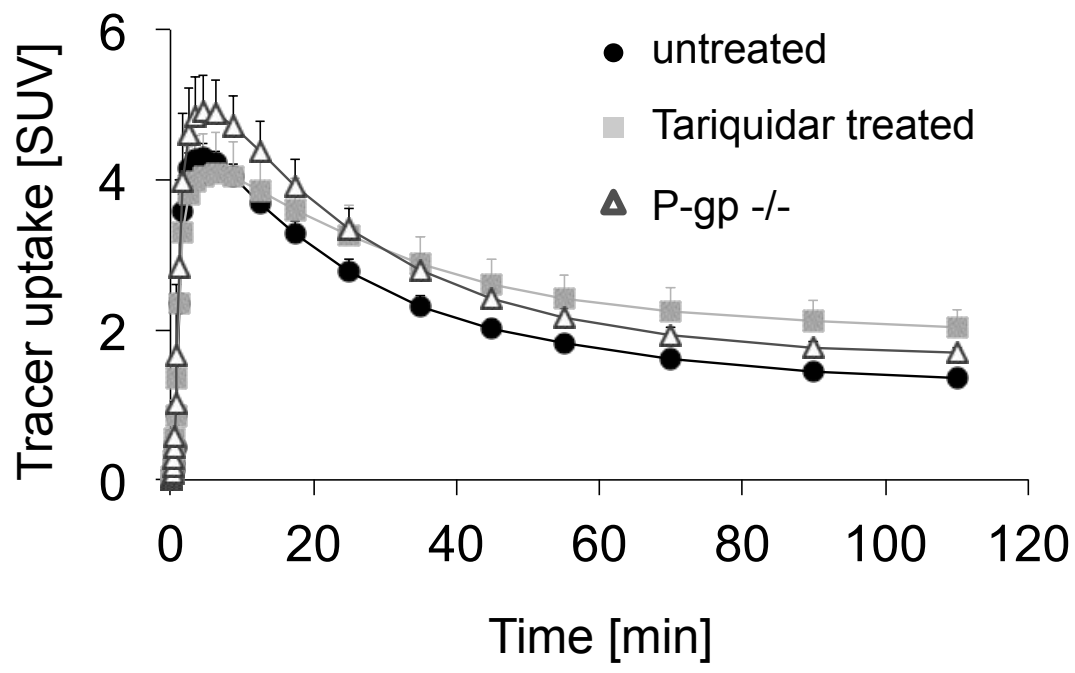

\begin{tabular}{l|lll} 
Group & $\begin{array}{l}\text { Efflux rate } \\
(1 / \mathrm{min})\end{array}$ & $\begin{array}{l}\text { Radioactivity: } \\
\text { washout / trapped }\end{array}$ \\
\hline Control & 0.0378 & 2.89 & \\
Tariquidar & 0.0333 & 1.50 & ACS Paragon \\
P-gp -/- & 0.0387 & 2.77 &
\end{tabular}

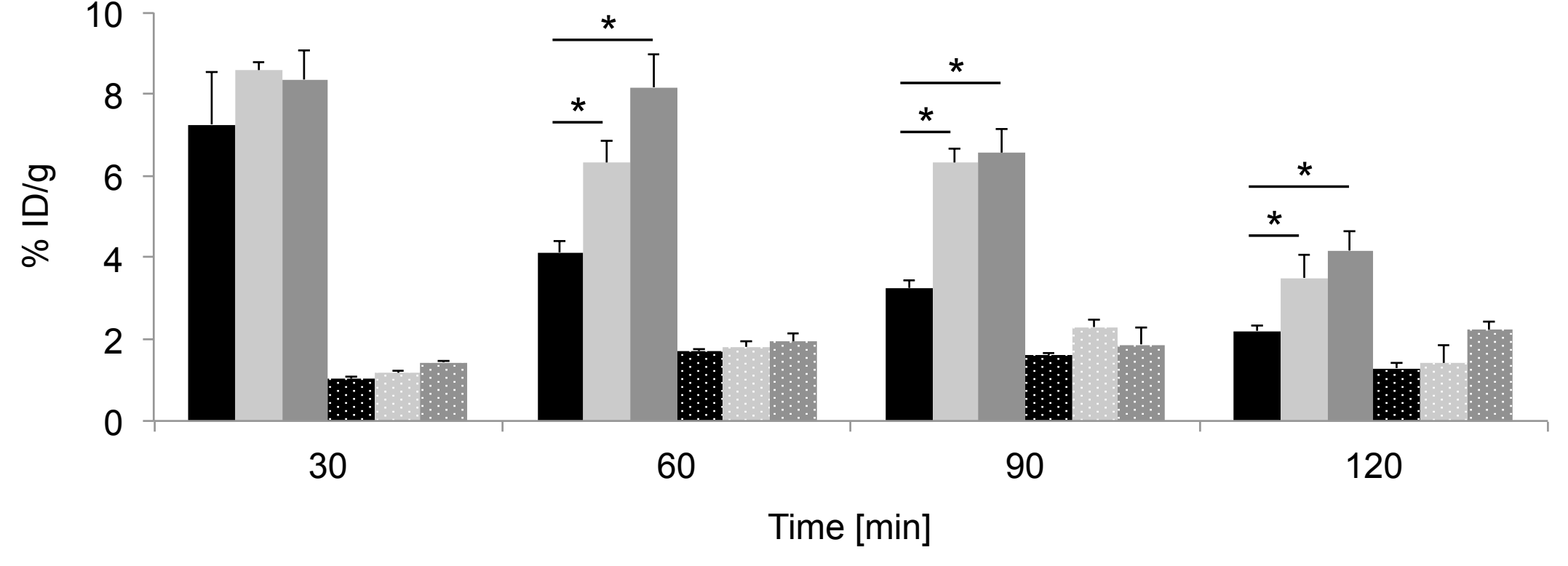

C

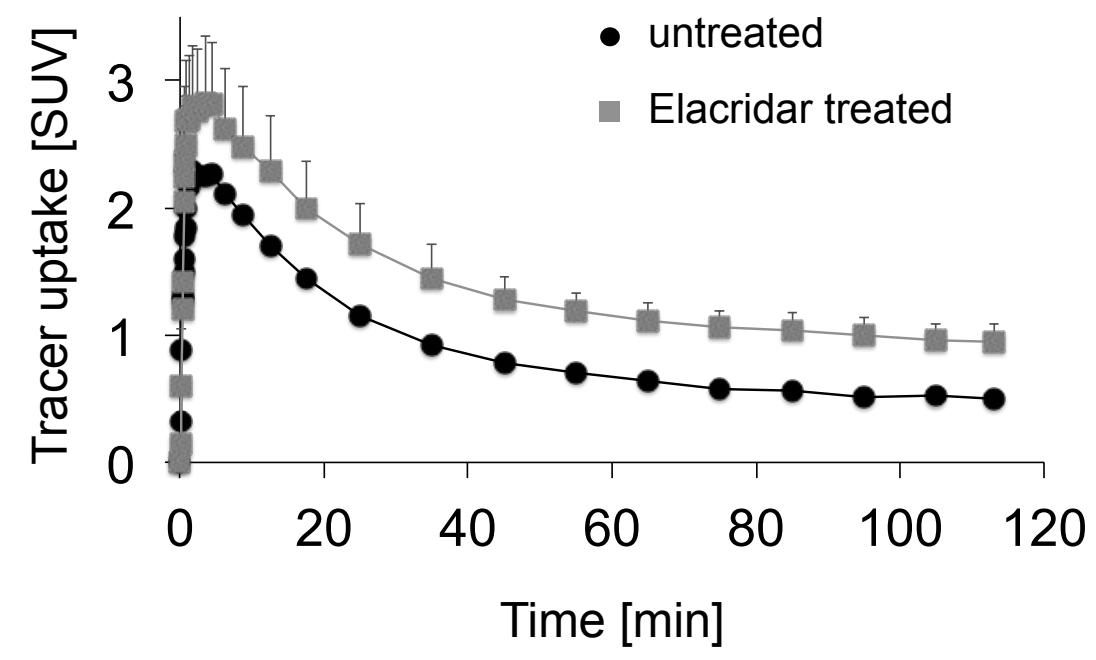

\begin{tabular}{l|ll} 
Group & $\begin{array}{l}\text { Efflux rate } \\
(1 / \mathrm{min})\end{array}$ & $\begin{array}{l}\text { Radioactivity: } \\
\text { washout / trapped }\end{array}$ \\
\hline Control & 0.0454 & 4.14 \\
$\begin{array}{l}\text { Elacridar } \\
\text { Environment }\end{array}$ & 0.0432 & 2.33
\end{tabular}




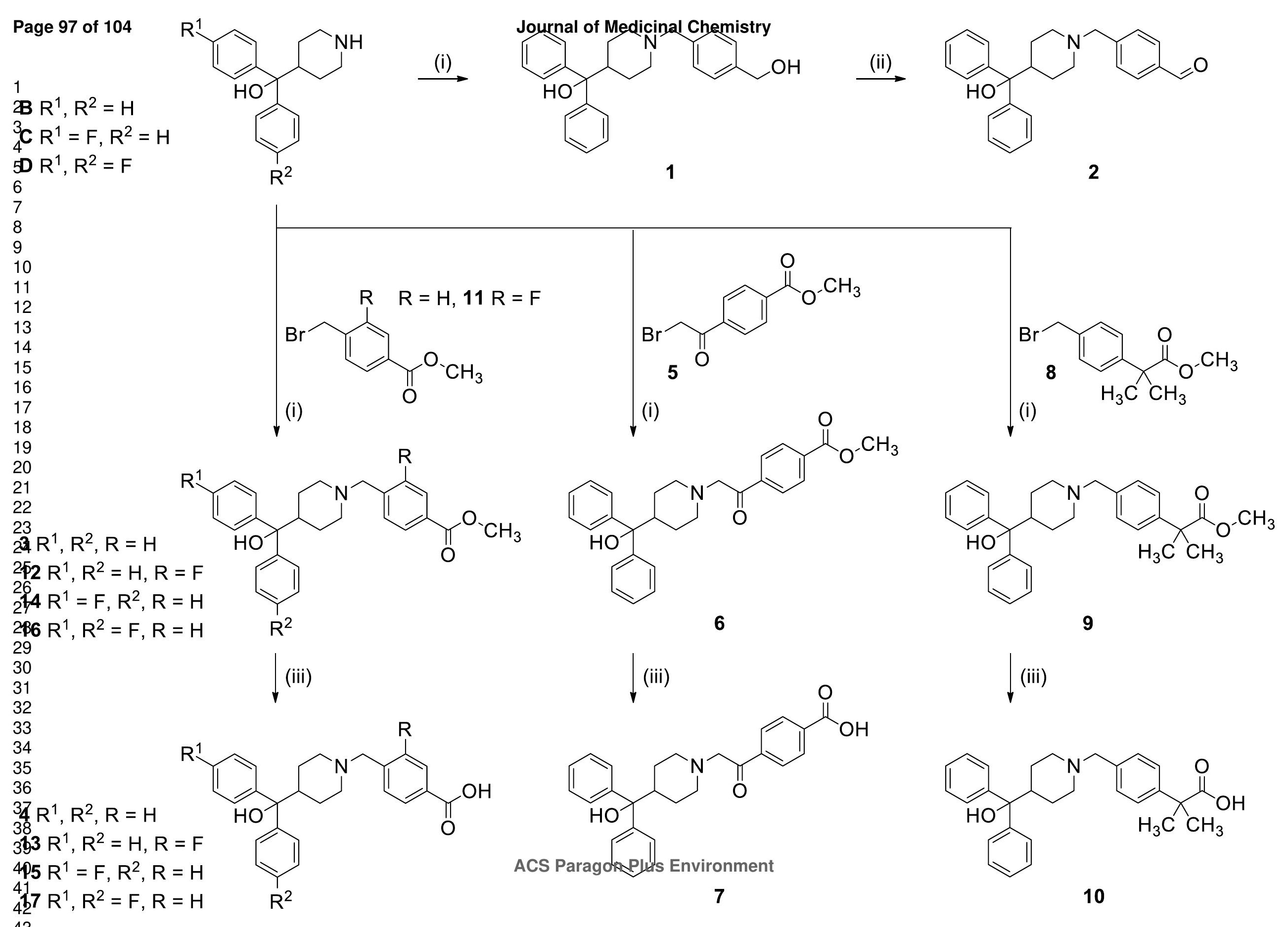




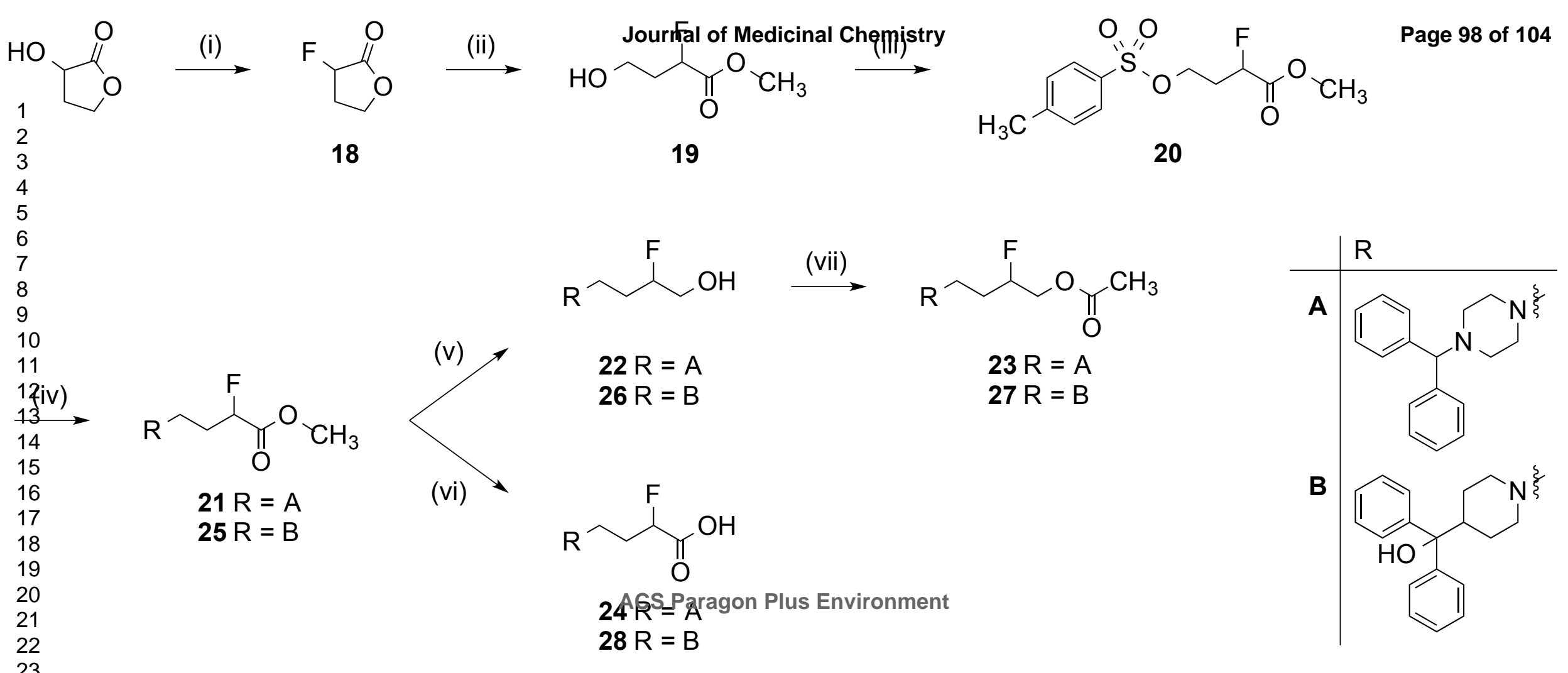




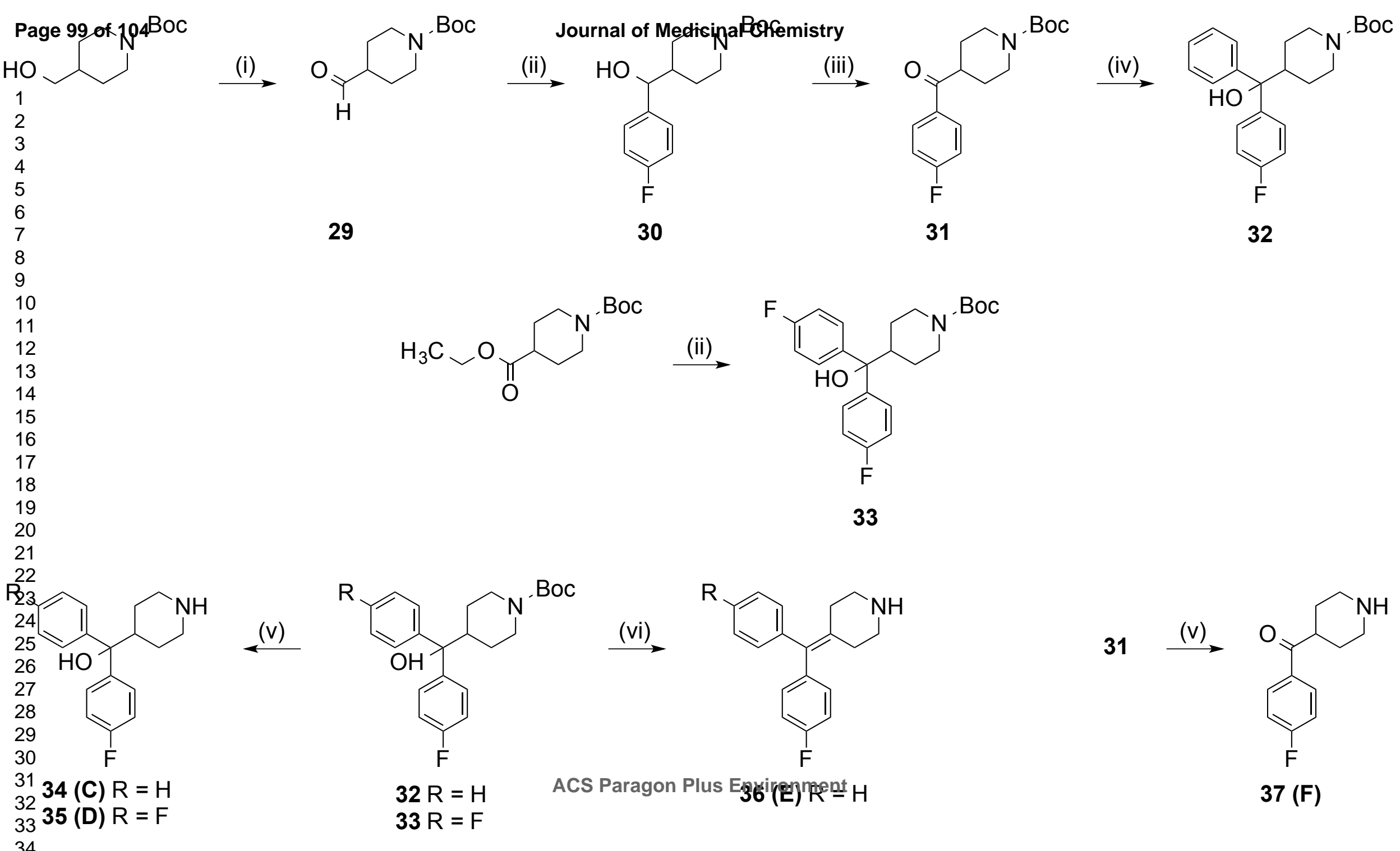




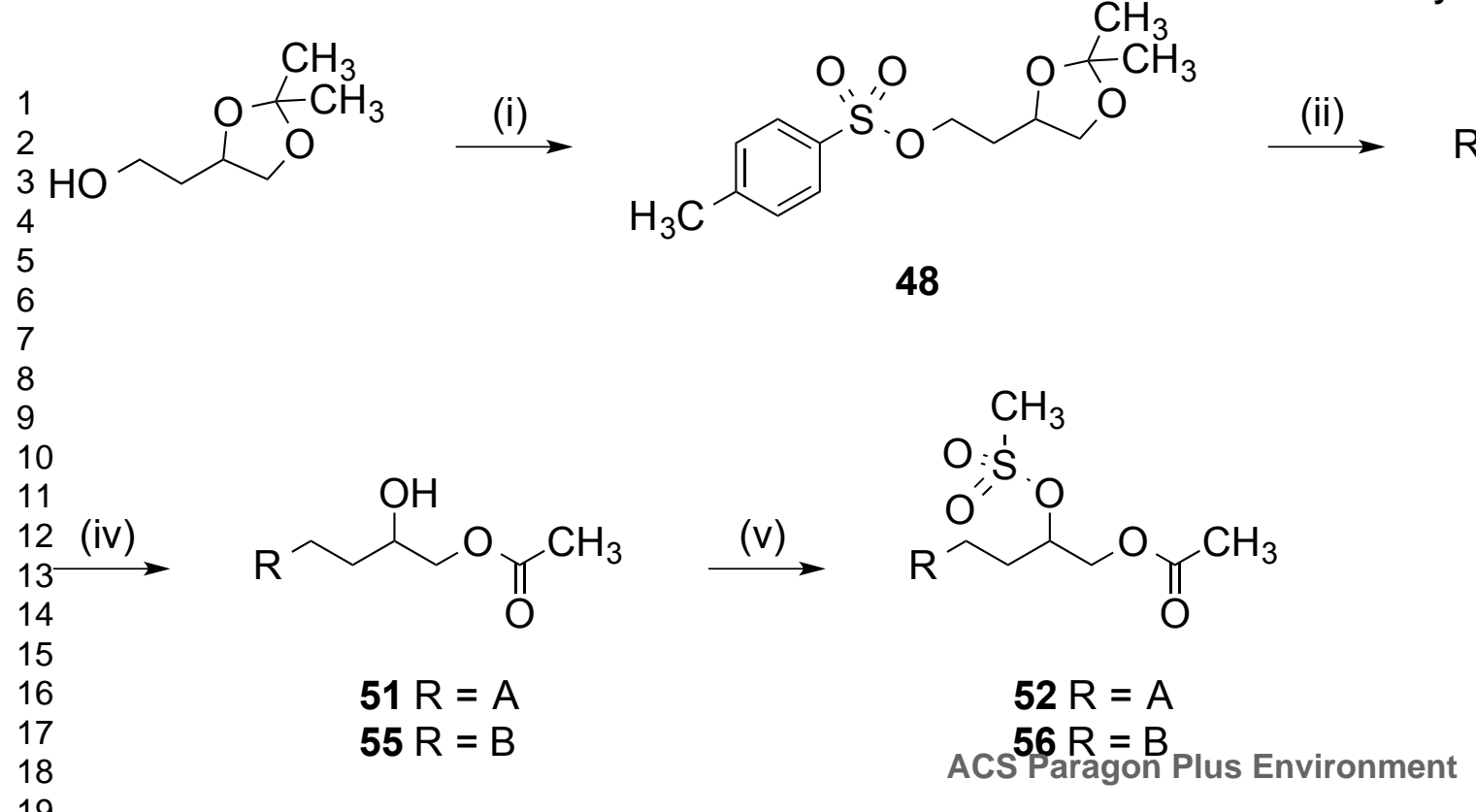

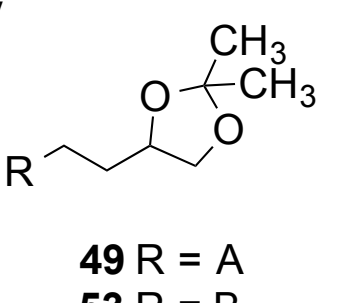

$53 \mathrm{R}=\mathrm{B}$ $\stackrel{\text { (iii) }}{\longrightarrow}$

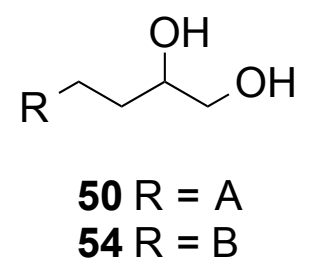

"Brat"




\section{Page 101 of 104}

Journal of Medicinal Chemistry

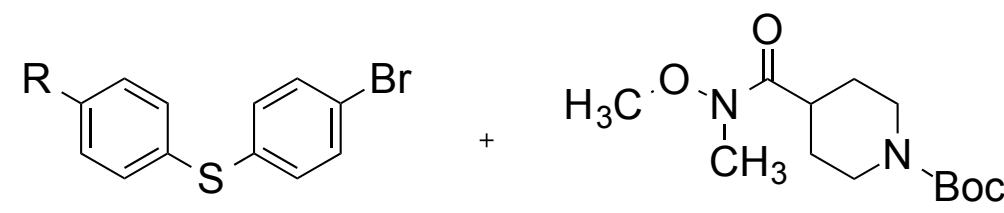

$57 \mathrm{R}=\mathrm{H}$

58

65 $\mathrm{R}=\mathrm{OCH}_{3}$

(i)
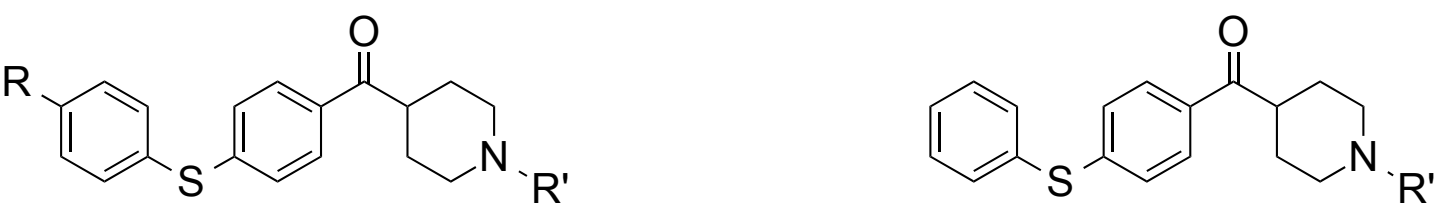

(ii) $C \begin{array}{rl}59 & \mathrm{R}=\mathrm{H}, \mathrm{R}^{\prime}=\mathrm{Boc} \\ 60 & \mathrm{R}=\mathrm{H}, \mathrm{R}^{\prime}=\mathrm{H}\end{array}$

$66 \mathrm{R}=\mathrm{OCH}_{3}, \mathrm{R}^{\prime}=\mathrm{Boc}$

$\downarrow(v)$
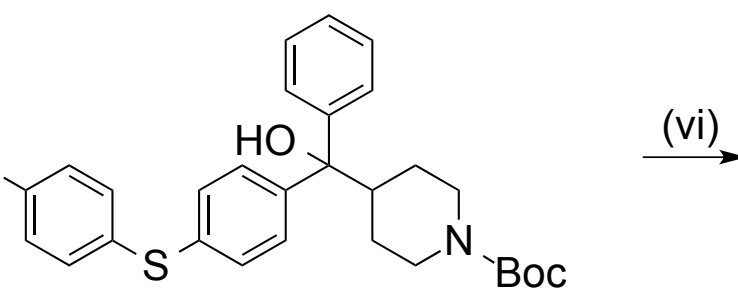

$\underline{67}$
$61,62 R^{\prime}=$

69,70

$\mathrm{R}^{\prime}=\stackrel{\mathrm{O}}{\prod_{\mathrm{O}}} \mathrm{CH}_{3}$

$63,64 \quad R^{\prime}=$

71,72

$73,74 \quad \mathrm{R}^{\prime}=$ ริ

$\stackrel{\text { (iv) }}{\longrightarrow}$

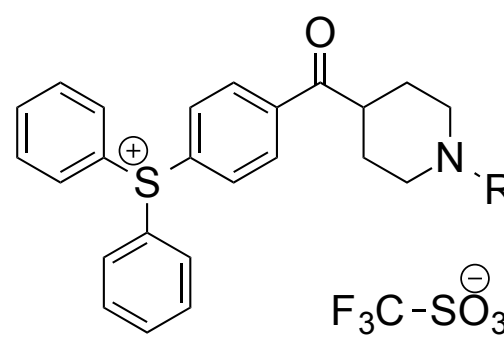

62,64

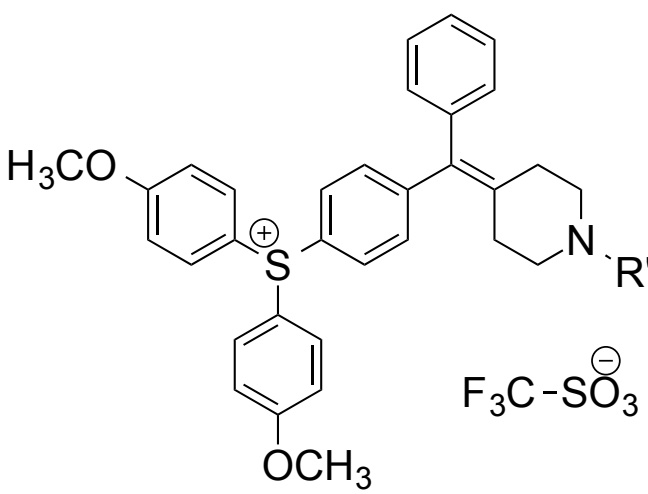

(vii)

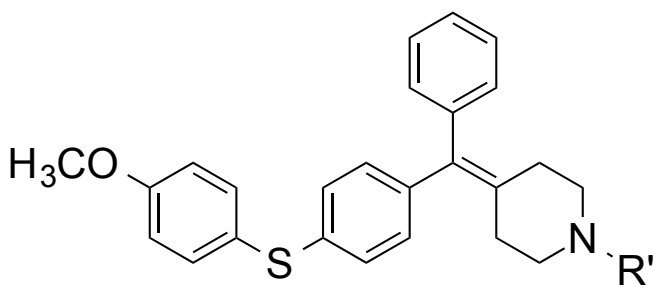

Acsipïaragon Plus Environment

$69,71,73$ 



\section{Page 103 $104 \mathrm{~F}_{3} \mathrm{C}-\mathrm{SO}_{3}^{\ominus}$}<smiles>[R]N1CCC(C(=O)c2ccc([Se](=O)c3ccccc3)cc2)CC1</smiles>

$\underline{62}, 64$ $\stackrel{\text { (i) }}{\longrightarrow}$<smiles>[CH]=[14CH][14CH]CCCOC(C)=O</smiles>

$\mathrm{R}^{\prime}=$

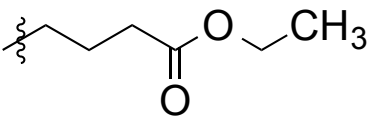

10

1162. $13\left[{ }^{18} \mathrm{~F}\right] \underline{45}$ 15 1664 , ${ }_{18}^{17}\left[{ }^{18} \mathrm{~F}\right] 47$ 19 20 21 22

Journal of Medicinal Chemistry<smiles>[R]N1CCC(C(=O)c2ccc([In+])cc2)CC1</smiles>

$\left[{ }^{18} \mathrm{~F}\right] \underline{45},\left[{ }^{18} \mathrm{~F}\right] 47$ $\stackrel{\text { (ii) }}{\longrightarrow}$ (iii)

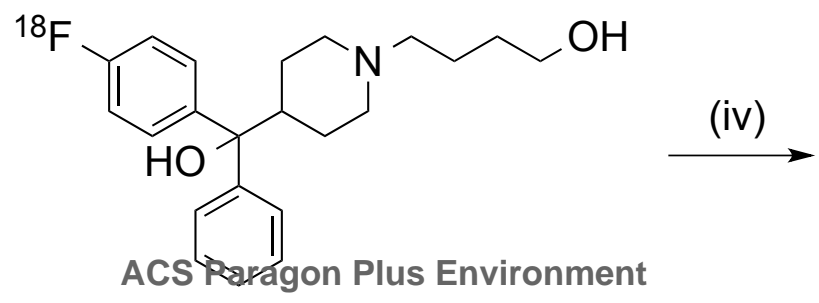

$\left[{ }^{18} \mathrm{~F}\right] \underline{39}$

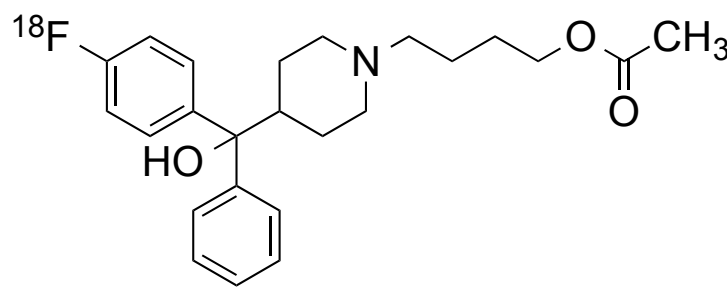

$\left[{ }^{18} \mathrm{~F}\right] 38$ 
$\mathrm{F}_{3} \mathrm{C}-\mathrm{SO}_{3}^{\ominus}$

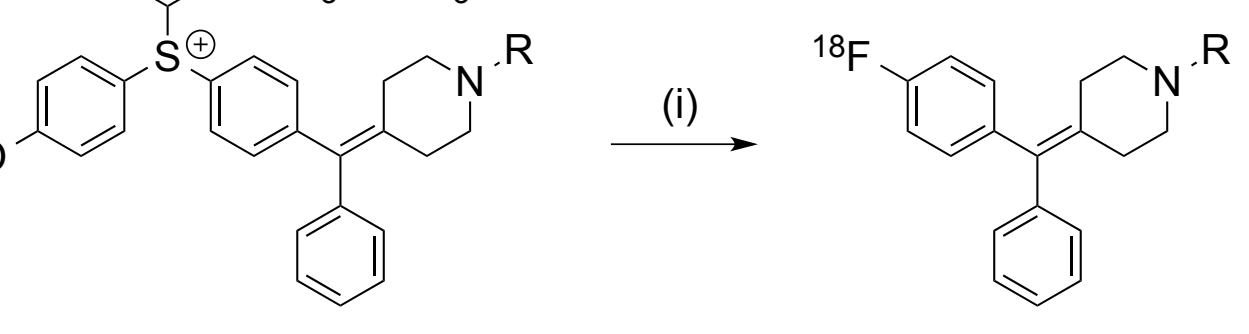

$70,\left[{ }^{18} \mathrm{~F}\right] 40 \mathrm{a} \quad \mathrm{R}=$ -

$72,\left[{ }^{18} \mathrm{~F}\right] 43 \quad \mathrm{R}=-\xi \overbrace{\mathrm{O}}^{\mathrm{O}} \sim \mathrm{CH}_{3}$ $74,\left[{ }^{18} \mathrm{~F}\right] 41$

ACS Parągon Plus Egvironment $\stackrel{\text { (ii) }}{\longrightarrow}\left[{ }^{18} \mathrm{~F}\right] 40 \mathrm{R}=$ -

$\stackrel{\text { (iii) }}{\longrightarrow}\left[{ }^{18} \mathrm{~F}\right] 44 \mathrm{R}=$-乡̧ $_{\mathrm{O}}^{\mathrm{OH}}$

$\stackrel{\text { (iv) }}{\longrightarrow}\left[{ }^{18} \mathrm{~F}\right] 42 \quad \mathrm{R}=$ Y
0<smiles>[3H]CCC=O</smiles> 


\section{Page 105@irtint of Medicinal Chemictru Blood}

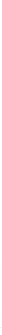

7

8 